\title{
Blow-up or no blow-up? A unified computational and analytic approach to 3D incompressible Euler and Navier-Stokes equations
}

\author{
Thomas Y. Hou \\ Applied and Computational Mathematics, 217-50, \\ Caltech, Pasadena, CA 91125, USA \\ E-mail: hou@acm.caltech.edu
}

Whether the 3D incompressible Euler and Navier-Stokes equations can develop a finite-time singularity from smooth initial data with finite energy has been one of the most long-standing open questions. We review some recent theoretical and computational studies which show that there is a subtle dynamic depletion of nonlinear vortex stretching due to local geometric regularity of vortex filaments. We also investigate the dynamic stability of the $3 \mathrm{D}$ Navier-Stokes equations and the stabilizing effect of convection. A unique feature of our approach is the interplay between computation and analysis. Guided by our local non-blow-up theory, we have performed large-scale computations of the 3D Euler equations using a novel pseudo-spectral method on some of the most promising blow-up candidates. Our results show that there is tremendous dynamic depletion of vortex stretching. Moreover, we observe that the support of maximum vorticity becomes severely flattened as the maximum vorticity increases and the direction of the vortex filaments near the support of maximum vorticity is very regular. Our numerical observations in turn provide valuable insight, which leads to further theoretical breakthrough. Finally, we present a new class of solutions for the 3D Euler and Navier-Stokes equations, which exhibit very interesting dynamic growth properties. By exploiting the special nonlinear structure of the equations, we prove nonlinear stability and the global regularity of this class of solutions. 


\section{CONTENTS}

1 Introduction

2 Dynamic depletion of vortex stretching in 3D Euler equations

3 Dynamic stability of 3D Navier-Stokes equations 305

4 Stabilizing effect of convection for 3D

Navier-Stokes

5 Concluding remarks

\section{Introduction}

The question of whether the 3D incompressible Navier-Stokes equations can develop a finite-time singularity from smooth initial data is one of the most long-standing open problems in fluid dynamics and mathematics. This is also one of the seven Millennium Open Problems posted by the Clay Mathematical Institute (see www.claymath.org). The understanding of this problem could improve our understanding on the onset of turbulence and the intermittency properties of turbulent flows.

The 3D incompressible Navier-Stokes equations are given by

$$
\begin{aligned}
\mathbf{u}_{t}+(\mathbf{u} \cdot \nabla) \mathbf{u} & =-\nabla p+\nu \Delta \mathbf{u}, \\
\nabla \cdot \mathbf{u} & =0,
\end{aligned}
$$

with initial condition $\mathbf{u}(\mathbf{x}, 0)=\mathbf{u}_{0}(\mathbf{x})$. Here $\mathbf{u}$ is velocity, $p$ is pressure, and $\nu$ is viscosity. We consider only the initial value problem and assume that the solution decays rapidly at infinity. Defining vorticity by $\boldsymbol{\omega}=\nabla \times \mathbf{u}$, then $\boldsymbol{\omega}$ is governed by

$$
\boldsymbol{\omega}_{t}+(\mathbf{u} \cdot \nabla) \boldsymbol{\omega}=\nabla \mathbf{u} \cdot \boldsymbol{\omega}+\nu \Delta \boldsymbol{\omega} .
$$

The first term on the right-hand side of (1.3) is called the vortex stretching term, which is absent in the two-dimensional problem. Note that $\nabla \mathbf{u}$ is formally of the same order as $\boldsymbol{\omega}$. Thus the vortex stretching term has a formal quadratic scaling with respect to vorticity. This formal quadratic nonlinearity in the vortex stretching term is the main difficulty in studying the dynamic stability and global regularity of the 3D Navier-Stokes equations. Under suitable smallness assumptions on the initial condition, global existence and regularity results have been obtained for some time (Ladyzhenskaya 1970, Constantin and Foias 1988, Temam 2001, Majda and Bertozzi 2002). But these methods based on energy estimates do not generalize to the 3D Navier-Stokes with large data. Energy estimates seem to be too crude to give a definite answer to whether diffusion is strong enough to control the nonlinear growth due to vortex stretching. A more refined 
analysis seems to be needed, which takes into account the special nature of the nonlinearities and their local interactions.

We believe that the global regularity of the 3D Navier-Stokes equations is closely related to that of the 3D Euler equations. Since the nonlinearity of the 3D Navier-Stokes equations is supercritical, the balance among different nonlinear terms in the Euler equations may play an even more important role than the diffusion term. Thus, it makes sense to investigate the mechanism which may lead to finite-time blow-up or dynamic depletion of the nonlinear vortex stretching in the 3D Euler equations.

There has been some interesting development in the theoretical understanding of the 3D incompressible Euler equations. In particular, Constantin, Fefferman and Majda have shown that the local geometric regularity of vortex lines can play an important role in depleting nonlinear vortex stretching (Constantin 1994, Constantin, Fefferman and Majda 1996). Inspired by their work, Deng, Hou and Yu $(2005,2006 a)$ recently showed that geometric regularity of vortex lines, even in an extremely localized region containing the maximum vorticity, can lead to depletion of nonlinear vortex stretching, thus avoiding finite-time singularity formation of the 3D Euler equations. To obtain these results, Deng, Hou and Yu used a Lagrangian approach and explored the connection between the stretching of local vortex lines and the growth of vorticity. In particular, they showed that if the vortex lines near the region of maximum vorticity satisfy some local geometric regularity conditions and the maximum velocity field is integrable in time, then no finite-time blow-up is possible. These localized non-blow-up criteria provide stronger constraints on the local geometry of a potential finite-time singularity.

There have been many computational attempts to find finite-time singularities of the 3D Euler and Navier-Stokes equations: see, e.g., Chorin (1982), Pumir and Siggia (1990), Kerr and Hussain (1989), Grauer and Sideris (1991), Shelley, Meiron and Orszag (1993), Kerr (1993), Caflisch (1993), Boratav and Pelz (1994), Fernandez, Zabusky and Gryanik (1995), Pelz (1997), Grauer, Marliani and Germaschewski (1998), Kerr (2005). One example that has been studied extensively is the interaction of two perturbed antiparallel vortex tubes. This example is interesting because of the vortex reconnection observed for the corresponding Navier-Stokes equations. It is natural to ask whether the 3D Euler equations would develop a finite-time singularity in the limit of vanishing viscosity. Kerr $(1993,2005)$ presented numerical evidence which suggested a finite-time singularity of the 3D Euler equations for two perturbed antiparallel vortex tubes. Kerr's blow-up scenario is consistent with the non-blow-up criterion of Beale, Kato and Majda (1984) and that of Constantin, Fefferman and Majda (1996). But it falls into the critical case of Deng, Hou and Yu's local non-blow-up criteria (Deng, Hou and Yu 2005, 2006a). 
Guided by this local geometric non-blow-up analysis, Hou and Li (2006) performed extremely large-scale computations with resolution up to $1536 \times$ $1024 \times 3072$ to re-examine Kerr's blow-up scenario (Kerr 1993). They used a novel pseudo-spectral method with a 36th-order Fourier smoothing function which keeps a significant portion of the Fourier modes beyond the $2 / 3$ cut-off point in the Fourier spectrum for the $2 / 3$ de-aliasing rule. Their extensive numerical results demonstrated that the pseudo-spectral method with the high-order Fourier smoothing gives a much better performance than the pseudo-spectral method with the $2 / 3$ de-aliasing rule. In particular, they showed that the Fourier smoothing method captures about $12 \sim 15 \%$ more effective Fourier modes than the $2 / 3$ de-aliasing method in each dimension. For 3D Euler equations, the total number of effective modes in the Fourier smoothing method is about $20 \%$ more than that in the $2 / 3$ de-aliasing method. This is a very significant increase in the resolution for a large-scale computation.

There were several interesting findings in the large-scale computations of Hou and Li (2006) for the 3D Euler equations using the initial data for the antiparallel vortex tubes. First, they discovered a surprising dynamic cancellation in the vortex stretching term due to the local geometric regularity of the vortex filaments. Vortex stretching was found to deplete dynamically from a formally quadratic nonlinearity to a much weaker $O(\omega \log (\omega))$ type of nonlinearity, which leads to only double exponential growth in the maximum vorticity. Secondly, they showed that the velocity field is bounded up to $T=19$, beyond the alleged singularity time $T=18.7$ of Kerr (2005). With a bounded velocity field, the non-blow-up criterion of Deng, Hou and $\mathrm{Yu}$ (2005) applies, which provides theoretical support for their computational results. Thirdly, they found that the vorticity vector near the point of maximum vorticity aligns almost perfectly with the second eigenvector of the rate of strain tensor. The second eigenvalue of the rate of strain tensor is the smallest eigenvalue and does not seem to grow dynamically, while the first and third eigenvalues grow very rapidly in time. This is further strong evidence for the dynamic depletion of vortex stretching.

Inspired by the numerical findings of their paper of 2006, Hou and Li (2008a) investigated the dynamic stability of the 3D Navier-Stokes equations by introducing an exact 1D model of the axisymmetric Navier-Stokes equations along the symmetry axis. This $1 \mathrm{D}$ model is exact in the sense that one can construct a family of exact solutions for the 3D Navier-Stokes equations from this 1D model. Thus the 1D model preserves some essential features of the 3D Navier-Stokes equations. What is surprising is that they obtained a Lyapunov function which satisfies a new maximum principle. This provides a pointwise estimate on the dynamic stability of the Navier-Stokes equations. The traditional energy estimates are incapable of capturing such subtle cancellation effects. Based on the global 
regularity of the $1 \mathrm{D}$ model, they constructed a new class of solutions for the 3D Euler and Navier-Stokes equations, which exhibit very interesting dynamic growth properties, but remain smooth for all times.

Motivated by the work of Hou and Li (2008a), Hou and Lei (2009b) further proposed a new 3D model to study the stabilizing effect of convection. This model was derived by neglecting the convection term from a reformulated axisymmetric Navier-Stokes equations. It shares almost all the properties of the 3D Navier-Stokes equations. In particular, the strong solution of the model satisfies an energy identity similar to that of the full 3D NavierStokes equations. They proved a non-blow-up criterion of Beale-KatoMajda type as well as a non-blow-up criterion of Prodi-Serrin type for the model. Moreover, they proved that, for any suitable weak solution of the 3D model in an open set in space-time, the one-dimensional Hausdorff measure of the associated singular set is zero (Hou and Lei 2009a). This partial regularity result is an analogue of the Caffarelli-Kohn-Nirenberg theory (Caffarelli, Kohn and Nirenberg 1982) for the 3D Navier-Stokes equations.

Despite the striking similarity at the theoretical level between the 3D model and the Navier-Stokes equations, the former has a completely different behaviour from the full Navier-Stokes equations. Hou and Lei's study showed that the 3D model seems to form a finite-time singularity, while the mechanism of generating such a finite-time singularity is removed when convection is added back to the 3D model. Convection seems to play a very important role in stabilizing the potential blow-up of the Navier-Stokes equations. This result may have an important impact on future global regularity analysis of 3D Navier-Stokes equations. Up to now, most analysis uses energy estimates in which convection plays no role at all. Such global methods of analysis are too crude. Their studies suggest that one needs to develop a new localized analysis which can in essence exploit the stabilizing effect of convection.

There has been some interesting development in the study of the 3D incompressible Navier-Stokes equations and related models. By exploiting the special structure of the governing equations, Cao and Titi (2007) proved the global well-posedness of the $3 \mathrm{D}$ viscous primitive equation for largescale ocean and atmospheric dynamics. For the axisymmetric Navier-Stokes equations, Chen, Strain, Tsai and Yau $(2008,2009)$ and Koch, Nadirashvili, Seregin and Sverak (2009) recently proved that if $|\mathbf{u}(x, t)| \leq C_{*}|t|^{-1 / 2}$, where $C_{*}$ is allowed to be large, then the velocity field $\mathbf{u}$ is regular at time zero. The 2D Boussinesq equations are closely related to the 3D axisymmetric Navier-Stokes equations with swirl (away from the symmetry axis). Recently, Hou and Li (2005) and Chae (2006) proved independently the global existence of the 2D Boussinesq equations with partial viscosity. By taking advantage of the limiting property of some rapidly oscillating operators and using nonlinear averaging, Babin, Mahalov and Nicolaenko (2001) proved 
global regularity of the 3D Navier-Stokes equations for some initial data characterized by uniformly large vorticity.

The rest of the paper is organized as follows. In Section 2, we study the dynamic depletion of vortex stretching for the 3D Euler equations. We also discuss at length how to design an effective high-resolution pseudospectral method to compute potentially singular solutions of the 3D Euler equations. Section 3 is devoted to studying the dynamic stability of the 3D Navier-Stokes equations. In Section 4, we investigate the stabilizing effect of convection for the 3D Navier-Stokes equations. Some concluding remarks are made in Section 5.

\section{Dynamic depletion of vortex stretching in 3D Euler equations}

Due to the supercritical nature of the nonlinearity of the 3D Navier-Stokes equations, the 3D Navier-Stokes equations with large initial data are convection-dominated, instead of diffusion-dominated. For this reason, we believe that the understanding of whether the corresponding 3D Euler equations would develop a finite-time blow-up could shed useful light on the global regularity of the Navier-Stokes equations.

Let us consider the 3D Euler equations in the vorticity form. One important observation is that when we consider the convection term together with the vortex stretching term, the two nonlinear terms can be actually represented as a commutator or a Lie derivative:

$$
\boldsymbol{\omega}_{t}+(\mathbf{u} \cdot \nabla) \boldsymbol{\omega}-(\boldsymbol{\omega} \cdot \nabla) \mathbf{u}=0 .
$$

It is reasonable to believe that the commutator would lead to some cancellation among the two nonlinear terms, thus weakening the nonlinearity dynamically. This points to the potential important role of convection in the 3D Euler equations. Another way to realize the importance of convection is to use the Lagrangian formulation of the vorticity equation. When we consider the two terms together, we preserve the Lagrangian structure of the solution (Chorin and Marsden 1993),

$$
\boldsymbol{\omega}(X(\alpha, t), t)=X_{\alpha}(\alpha, t) \boldsymbol{\omega}_{0}(\alpha),
$$

where $X_{\alpha}=\frac{\partial X}{\partial \alpha}$ and $X(\alpha, t)$ is the flow map,

$$
\frac{\mathrm{d} X}{\mathrm{~d} t}(\alpha, t)=\mathbf{u}(X(\alpha, t), t), \quad X(\alpha, 0)=\alpha .
$$

Therefore, vorticity increases in time only through the dynamic deformation of the Lagrangian flow map. On the other hand, due to the divergence-free property of the velocity field, the flow map is volume-preserving, that is, 
$\operatorname{det}\left(X_{\alpha}(\alpha, t)\right) \equiv 1$. Thus, as vorticity increases dynamically, the parallelepiped spanned by the three vectors, $\left(X_{\alpha_{1}}, X_{\alpha_{2}}, X_{\alpha_{3}}\right)$, will experience severe deformation and become flattened dynamically. A formal asymptotic analysis shows that the support of maximum vorticity also experiences a similar deformation and becomes severely flattened as vorticity increases. This is confirmed by our numerical experiments: see Section 2.5. Such deformation tends to weaken the nonlinearity of vortex stretching dynamically.

We remark that convection plays an essential role in deforming the support of maximum vorticity and induces an anisotropic scaling in the collapse of the support of maximum vorticity. By exploiting the anisotropic scaling of the support of maximum vorticity, Hou, Lei and Li (2008) recently proved the global regularity of the axisymmetric Navier-Stokes equations with a family of very large anisotropic initial data: see Section 2.8 for more discussions. On the other hand, if we ignore the convection term in the Euler equations, the vortex stretching term may indeed achieve the $O\left(|\omega|^{2}\right)$ scaling dynamically and develop an isotropic singularity in finite time: see Section 4 for more discussions.

\subsection{A brief review}

We begin with a brief review of the subject. Due to the formal quadratic nonlinearity in vortex stretching, only short time existence is known for the 3D Euler equations (Majda and Bertozzi 2002). One of the most well-known results on the 3D Euler equations is due to Beale, Kato and Majda (1984), who showed that the solution of the 3D Euler equations blows up at $T$ if and only if $\int_{0}^{T}\|\boldsymbol{\omega}\|_{\infty}(t) \mathrm{d} t=\infty$, where $\boldsymbol{\omega}$ is vorticity.

There have been some interesting recent theoretical developments. In particular, Constantin, Fefferman and Majda (1996) showed that local geometric regularity of the unit vorticity vector can lead to depletion of the vortex stretching. Let $\boldsymbol{\xi}=\boldsymbol{\omega} /|\boldsymbol{\omega}|$ be the unit vorticity vector and let $\mathbf{u}$ be the velocity field. Roughly speaking, Constantin, Fefferman and Majda proved that if (1) $\|\mathbf{u}\|_{\infty}$ is bounded in a $O(1)$ region containing the maximum vorticity, (2) $\int_{0}^{t}\|\nabla \boldsymbol{\xi}\|_{\infty}^{2} \mathrm{~d} \tau$ is uniformly bounded for $t<T$, then the solution of the 3D Euler equations remains regular up to $t=T$.

There has been some numerical evidence that suggests a finite-time blowup of the 3D Euler equations. One of the most well-known examples is the finite-time collapse of two antiparallel vortex tubes by R. Kerr (1993, 2005). In his computations, Kerr used a pseudo-spectral discretization in the $x$ - and $y$-directions, and a Chebyshev discretization in the $z$-direction with resolution of order $512 \times 256 \times 192$. His computations showed that the maximum vorticity blows up like $O\left((T-t)^{-1}\right)$ with $T=18.9$. In his subsequent paper, Kerr (2005) applied a high wavenumber filter to the data obtained in his original computations to 'remove the noise that masked the 
structures in earlier graphics' presented in the 1993 paper. With this filtered solution, he presented some scaling analysis of the numerical solutions up to $t=17.5$. Two new properties were presented in the 2005 paper. First, the velocity field was shown to blow up like $O(T-t)^{-1 / 2}$ with $T$ being revised to $T=18.7$. Secondly, he showed that the blow-up is characterized by two anisotropic length scales, $\rho \approx(T-t)$ and $R \approx(T-t)^{1 / 2}$. It is worth noting that there is still a considerable gap between the predicted singularity time $T=18.7$ and the final time $t=17$ of Kerr's original computations, which he used as the primary evidence for the finite-time singularity.

Kerr's blow-up scenario is consistent with the non-blow-up criterion of Beale, Kato and Majda (1984) and that of Constantin, Fefferman and Majda (1996). But it falls into the critical case of Deng, Hou and Yu's local nonblow-up criteria (Deng, Hou and Yu 2005, 2006a). Below we describe the local non-blow-up criteria of Deng, Hou and Yu.

\subsection{The local non-blow-up criteria of Deng, Hou and Yu (2005, 2006a)}

Motivated by the result of Constantin, Fefferman and Majda (1996), Deng, Hou and Yu (2005) have obtained a sharper non-blow-up condition which uses only very localized information of the vortex lines. Assume that at each time $t$ there exists some vortex line segment $L_{t}$ on which the local maximum vorticity is comparable to the global maximum vorticity. Further, we denote $L(t)$ as the arclength of $L_{t}, \mathbf{n}$ the unit normal vector of $L_{t}$, and $\kappa$ the curvature of $L_{t}$.

Theorem 2.1. (Deng, Hou and Yu 2005) Assume that (1) $\max _{L t}(\mid \mathbf{u}$. $\boldsymbol{\xi}|+| \mathbf{u} \cdot \mathbf{n} \mid) \leq C_{U}(T-t)^{-A}$ with $A<1$, and $(2) C_{L}(T-t)^{B} \leq L(t) \leq$ $C_{0} / \max _{L_{t}}(|\kappa|,|\nabla \cdot \boldsymbol{\xi}|)$ for $0 \leq t<T$. Then the solution of the 3D Euler equations remains regular up to $t=T$ provided that $A+B<1$.

In Kerr's computations, the first condition of Theorem 2.1 is satisfied with $A=1 / 2$ if we use $\|\mathbf{u}\|_{\infty} \leq C(T-t)^{-1 / 2}$ as alleged in Kerr (2005). Kerr's computations suggested that $\kappa$ and $\nabla \cdot \boldsymbol{\xi}$ are bounded by $O\left((T-t)^{-1 / 2}\right)$ in the inner region of size $(T-t)^{1 / 2} \times(T-t)^{1 / 2} \times(T-t)$ (Kerr 2005). Moreover, the length of the vortex tube in the inner region is of order $(T-t)^{1 / 2}$. If we choose a vortex line segment of length $(T-t)^{1 / 2}$ (i.e., $\left.B=1 / 2\right)$, then the second condition is satisfied. However, we violate the condition $A+B<1$. Thus Kerr's computations fall into the critical case of Theorem 2.1. In a subsequent paper, Deng, Hou and Yu (2006a) improved the non-blow-up condition to include the critical case, $A+B=1$.

Theorem 2.2. (Deng, Hou and Yu 2006a) Under the same assumptions as Theorem 2.1, in the case of $A+B=1$, the solution of the 3D Euler equations remains regular up to $t=T$ if the scaling constants $C_{U}, C_{L}$ and $C_{0}$ satisfy an algebraic inequality, $f\left(C_{U}, C_{L}, C_{0}\right)>0$. 
We remark that this algebraic inequality can be checked numerically if we obtain a good estimate of these scaling constants. For example, if $C_{0}=$ 0.1 , which seems reasonable since the vortex lines are relatively straight in the inner region, Theorem 2.2 would imply no blow-up up to $T$ if $2 C_{U}<$ $0.43 C_{L}$. Unfortunately, there was no estimate available for these scaling constants in Kerr (1993). One of our original motivations for repeating Kerr's computations using higher resolutions was to obtain a good estimate for these scaling constants.

\subsection{Computing potentially singular solutions using pseudo-spectral methods}

Computing Euler singularities numerically is an extremely challenging task. First of all, it requires huge computational resources. Tremendous resolutions are required to capture the nearly singular behaviour of the Euler equations. Secondly, one has to perform a careful convergence study. It is dangerous to interpret the blow-up of an under-resolved computation as evidence of finite-time singularities for the 3D Euler equations. Thirdly, if we believe that the numerical solution we compute leads to a finite-time blowup, we need to demonstrate the validity of the asymptotic blow-up rate, i.e., is the blow-up rate $\|\boldsymbol{\omega}\|_{L^{\infty}} \approx \frac{C}{(T-t)^{\alpha}}$ asymptotically valid as $t \rightarrow T$ ? If a numerical solution is well resolved only up to $T_{0}$ and there is still an order-one gap between $T_{0}$ and the predicted singularity time $T$, then one can not apply the Beale-Kato-Majda criterion (Beale, Kato and Majda 1984) to this fitted singularity, since the most significant contribution to $\int_{0}^{T}\|\boldsymbol{\omega}(t)\|_{L^{\infty}} \mathrm{d} t$ comes from the time interval $\left[T_{0}, T\right]$, but there is no accuracy in the extrapolated solution in this time interval if $\left(T-T_{0}\right)=O(1)$. Finally, one also needs to check if the blow-up rate of the numerical solution is consistent with other non-blow-up criteria (Constantin, Fefferman and Majda 1996, Deng, Hou and Yu 2005, Deng, Hou and Yu 2006a) which provide additional constraints on the blow-up rate of the velocity field and the local geometric regularity on the vortex filaments. The interplay between theory and numerics is clearly essential in our search for Euler singularities.

Hou and Li $(2006,2007)$ repeated Kerr's computations using two pseudospectral methods. The first pseudo-spectral method used the standard $2 / 3$ de-aliasing rule to remove the aliasing error. For the second pseudo-spectral method, they used a novel 36th-order Fourier smoothing to remove the aliasing error. For the Fourier smoothing method, they used a Fourier smoother along the $x_{j}$-direction as follows: $\rho\left(2 k_{j} / N_{j}\right) \equiv \exp \left(-36\left(2 k_{j} / N_{j}\right)^{36}\right)$, where $k_{j}$ is the wavenumber $\left(\left|k_{j}\right| \leq N_{j} / 2\right)$. The time integration was performed by using the classical fourth-order Runge-Kutta scheme. Adaptive timestepping was used to satisfy the CFL stability condition with CFL number equal to $\pi / 4$. In order to perform a careful resolution study, they 
used a sequence of resolutions: $768 \times 512 \times 1536,1024 \times 768 \times 2048$ and $1536 \times 1024 \times 3072$ in their computations. They computed the solution up to $t=19$, beyond the alleged singularity time $T=18.7$ by Kerr $(2005)$. Their computations were carried out on the PC cluster LSSC-II in the Institute of Computational Mathematics and Scientific/Engineering Computing of Chinese Academy of Sciences and the Shenteng 6800 cluster in the Super Computing Center of the Chinese Academy of Sciences. The maximal memory consumption in their computations was about 120 Gbytes. The largest number of grid points is close to 5 billion.

\subsection{Convergence study of spectral methods for the Burgers equation}

As a first step, we demonstrate that the two pseudo-spectral methods can be used to compute a singular solution arbitrarily close to the singularity time. For this purpose, we perform a careful convergence study of the two pseudo-spectral methods in both physical and spectral spaces for the 1D inviscid Burgers equation. The advantage of using the inviscid 1D Burgers equation is that it shares some essential difficulties with the 3D Euler equations, yet we have a semi-analytic formulation for its solution. By using the Newton iterative method, we can obtain an approximate solution to the exact solution up to 13 digits of accuracy. Moreover, we know exactly when a shock singularity will form in time. This enables us to perform a careful convergence study in both physical space and spectral space very close to the singularity time. This provides a solid foundation to the convergence study of the two spectral methods.

We consider the inviscid 1D Burgers equation

$$
u_{t}+\left(\frac{u^{2}}{2}\right)_{x}=0, \quad-\pi \leq x \leq \pi,
$$

with an initial condition given by

$$
\left.u\right|_{t=0}=u_{0}(x) .
$$

We impose a periodic boundary condition over $[-\pi, \pi]$. By the method of characteristics, it is easy to show that the solution of the 1D Burgers equation is given by

$$
u(x, t)=u_{0}(x-t u(x, t)) .
$$

The above implicit formulation defines a unique solution for $u(x, t)$ up to the time when the first shock singularity develops. After the shock singularity develops, equation (2.5) gives a multi-valued solution. An entropy condition is required to select a unique physical solution beyond the shock singularity (LeVeque 1992).

We now use a standard pseudo-spectral method to approximate the solution. Let $N$ be an integer, and let $h=\pi / N$. We denote by $x_{j}=j h$ 
$(j=-N, \ldots, N)$ the discrete grid points over the interval $[-\pi, \pi]$. To describe the pseudo-spectral methods, we recall that the discrete Fourier transform of a periodic function $u(x)$ with period $2 \pi$ is defined by

$$
\hat{u}_{k}=\frac{1}{2 N} \sum_{j=-N+1}^{N} u\left(x_{j}\right) \mathrm{e}^{-\mathrm{i} k x_{j}} .
$$

The inversion formula reads

$$
u\left(x_{j}\right)=\sum_{k=-N+1}^{N} \hat{u}_{k} \mathrm{e}^{\mathrm{i} k x_{j}} .
$$

We note that $\hat{u}_{k}$ is periodic in $k$ with period $2 N$. This is an artifact of the discrete Fourier transform, and the source of the aliasing error. To remove the aliasing error, one usually applies some kind of de-aliasing filtering when we compute the discrete derivative. Let $\rho(k / N)$ be a cut-off function in the spectrum space. A discrete derivative operator may be expressed in the Fourier transform as

$$
{\widehat{\left(D_{h} u\right.}}_{k}=\mathrm{i} k \rho(k / N) \widehat{u}_{k}, \quad k=-N+1, \ldots, N .
$$

Both the $2 / 3$ de-aliasing rule and the Fourier smoothing method can be described by a specific choice of the high-frequency cut-off function, $\rho$ (also known as Fourier filter). For the $2 / 3$ de-aliasing rule, the cut-off function is chosen to be

$$
\rho(k / N)= \begin{cases}1, & \text { if }|k / N| \leq 2 / 3, \\ 0, & \text { if }|k / N|>2 / 3 .\end{cases}
$$

In our computations, in order to obtain an alias-free computation on a grid of $M$ points for a quadratic nonlinear equation, we apply the above filter to the high wavenumbers so as to retain only $(2 / 3) M$ unfiltered wavenumbers before making the coefficient-to-grid Fast Fourier Transform. This dealiasing procedure is alternatively known as the $3 / 2$ de-aliasing rule because to obtain $M$ unfiltered wavenumbers one must compute nonlinear products in physical space on a grid of (3/2) $M$ points: see p. 229 of Boyd (2000) for more discussions.

For the Fourier smoothing method, we choose $\rho$ as follows:

$$
\rho(k / N)=\mathrm{e}^{-\alpha(|k| / N)^{m}},
$$

with $\alpha=36$ and $m=36$. In our implementation, both filters are applied to the numerical solution at every time step. Thus, for the $2 / 3$ de-aliasing rule, the Fourier modes with wavenumbers $|k| \geq 2 / 3 N$ are always set to zero. Thus there is no aliasing error being introduced in our approximation of the nonlinear convection term. For the Fourier smoothing method, the nonlinear term will have some non-zero modes beyond the $2 / 3$ point cut-off 


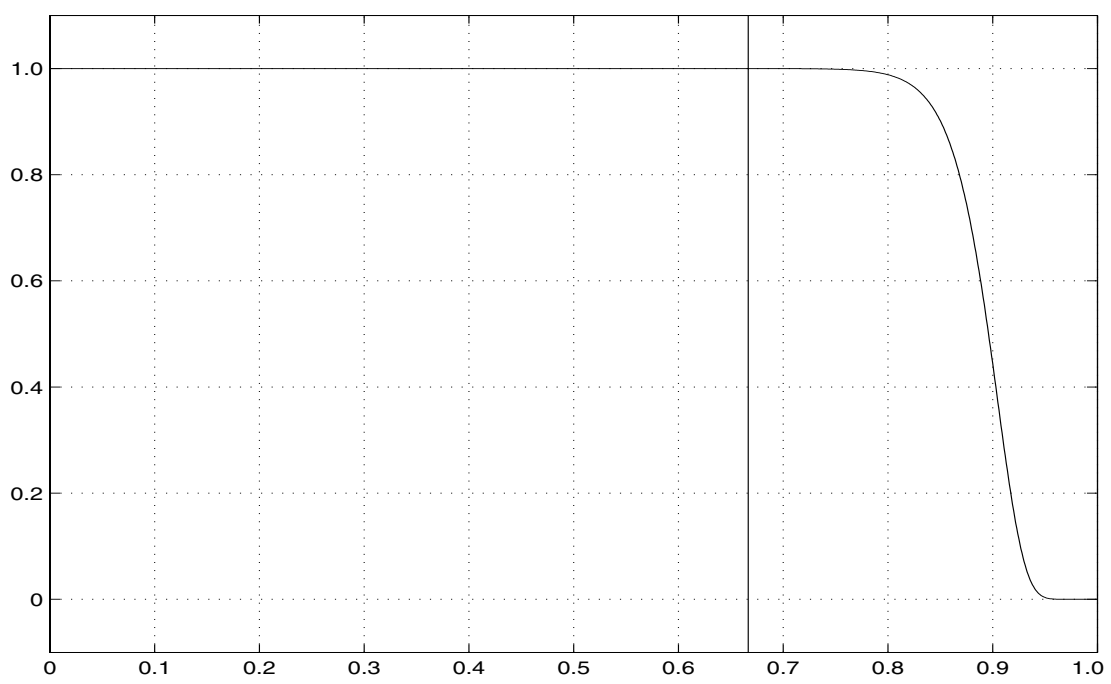

Figure 2.1. The profile of the Fourier smoothing, $\exp \left(-36(x)^{36}\right)$, as a function of $x$. The vertical line corresponds to the cut-off point in the Fourier spectrum in the $2 / 3$ de-aliasing rule. We can see that using this Fourier smoothing we keep about $12 \sim 15 \%$ more modes than those using the $2 / 3$ de-aliasing rule.

point in the Fourier space. However, these non-zero modes will accumulate in time to pollute the solution.

The Fourier smoothing method we choose is based on three considerations. The first one is that the aliasing instability is introduced by the highest-frequency Fourier modes. As demonstrated in Goodman, Hou and Tadmor (1994), as long as one can damp out a small portion of the highestfrequency Fourier modes, the mild instability caused by the aliasing error can be under control. The second observation is that the magnitude of the Fourier coefficient is decreasing with respect to the wavenumber $|k|$ for a function that has a certain degree of regularity. Typically, we have $\left|\widehat{u}_{k}\right| \leq C /\left(1+|k|^{m}\right)$ if the $m$ th derivative of a function $u$ is bounded in $L^{1}$. Thus the high-frequency Fourier modes have a relatively smaller contribution to the overall solution than the low- to intermediate-frequency modes. The third observation is that one should not cut off high-frequency Fourier modes abruptly to avoid the Gibbs phenomenon and the loss of the $L^{2}$ energy associated with the solution. This is especially important when we compute a nearly singular solution whose high-frequency Fourier coefficient has a very slow decay.

Based on the above considerations, we choose a smooth cut-off function which decays exponentially fast with respect to the high wavenumber. In our cut-off function, we choose the parameters $\alpha=36$ and $m=36$. These 
two parameters are chosen to achieve two objectives. (i) When $|k|$ is close to $N$, the cut-off function reaches machine precision, i.e., $10^{-16}$. (ii) The cut-off function remains very close to 1 for $|k|<4 N / 5$, and decays rapidly and smoothly to zero beyond $|k|=4 N / 5$. In Figure 2.1, we plot the cutoff function $\rho(x)$ as a function of $x$. The cut-off function used by the $2 / 3$ de-aliasing rule is plotted on top of the cut-off function used by the Fourier smoothing method. We can see that the Fourier smoothing method keeps about $12 \sim 15 \%$ more modes than the $2 / 3$ de-aliasing method. In this paper, we will demonstrate by our numerical experiments that the extra modes we keep by the Fourier smoothing method give an accurate approximation of the correct high-frequency Fourier modes.

We have performed a sequence of resolution studies with the largest resolution being $N=16384$ (Hou and Li 2007). Our extensive numerical results demonstrate that the pseudo-spectral method with the high-order Fourier smoothing (the Fourier smoothing method for short) gives a much more accurate approximation than the pseudo-spectral method with the $2 / 3$ dealiasing rule (the $2 / 3$ de-aliasing method for short). One of the interesting observations is that the unfiltered high-frequency coefficients in the Fourier smoothing method approximate accurately the corresponding exact Fourier coefficients. Moreover, we observe that the Fourier smoothing method captures about $12 \sim 15 \%$ more effective Fourier modes than the $2 / 3$ de-aliasing method in each dimension: see Figure 2.2. The gain is even higher for the 3D Euler equations since the number of effective modes in the Fourier smoothing method is higher in three dimensions. Further, we find that the error produced by the Fourier smoothing method is highly localized near the region where the solution is most singular. In fact, the pointwise error decays exponentially fast away from the location of the shock singularities. On the other hand, the error produced by the $2 / 3$ de-aliasing method spreads out to the entire domain as we approach the singularity time: see Figure 2.3.

\subsection{The high-resolution 3D Euler computations of Hou and Li (2006, 2007)}

Hou and Li (2006) performed high-resolution computations of the 3D Euler equations using the initial data for the two antiparallel vortex tubes. They used the same initial condition whose analytic formula was given by Kerr (see Section III of Kerr (1993), and also Hou and Li (2006) for corrections of some typos in the description of the initial condition in Kerr (1993)). However, there was some minor difference between their discretization and Kerr's discretization. Hou and Li used a pseudo-spectral discretization in all three directions, while Kerr used a pseudo-spectral discretization only in the $x$ - and $y$-directions and used a Chebyshev discretization in the $z$-direction. Based on the results of early tests, positive vorticity in the symmetry plane was imposed in the initial condition of Kerr (1993). How this was imposed 
(a)

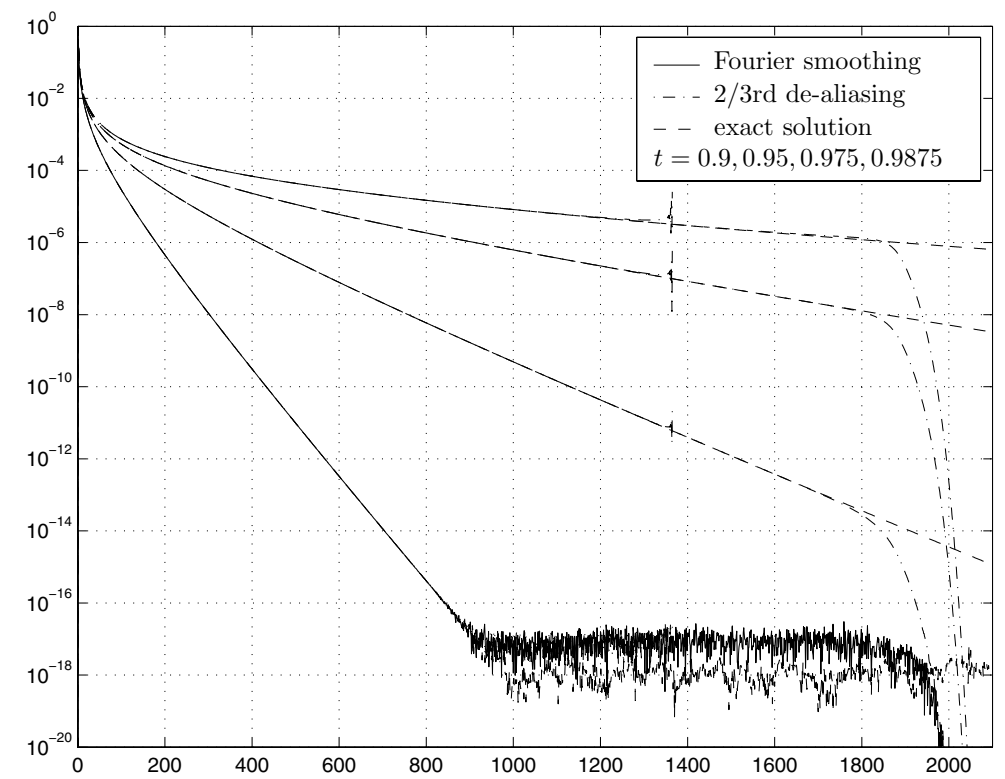

(b)

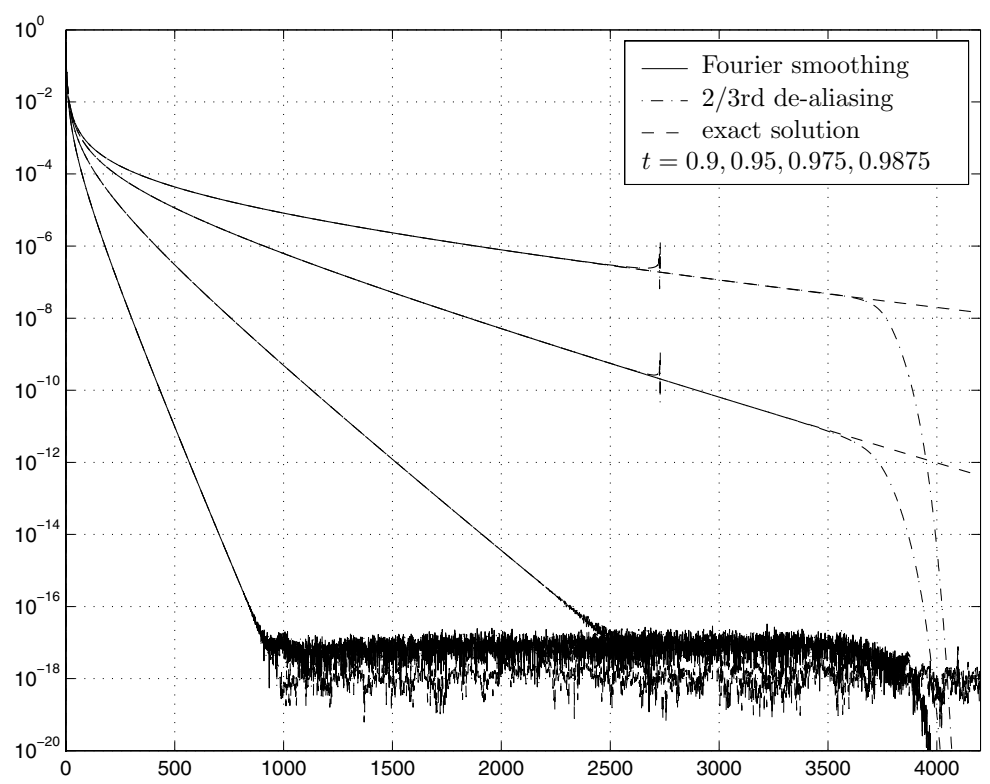

Figure 2.2. Comparison of Fourier spectra of the two methods on different resolutions at a sequence of times. (a) $N=4096$, (b) $N=8192$. Dashed lines, 'exact' spectra; solid lines, Fourier smoothing method; dash-dotted lines, $2 / 3$ de-aliasing method. Times, $t=0.9,0.95,0.975,0.9875$ respectively (from bottom to top). Initial condition, $u_{0}(x)=\sin (x)$. Singularity time for this initial condition, $T=1$. 
(a)

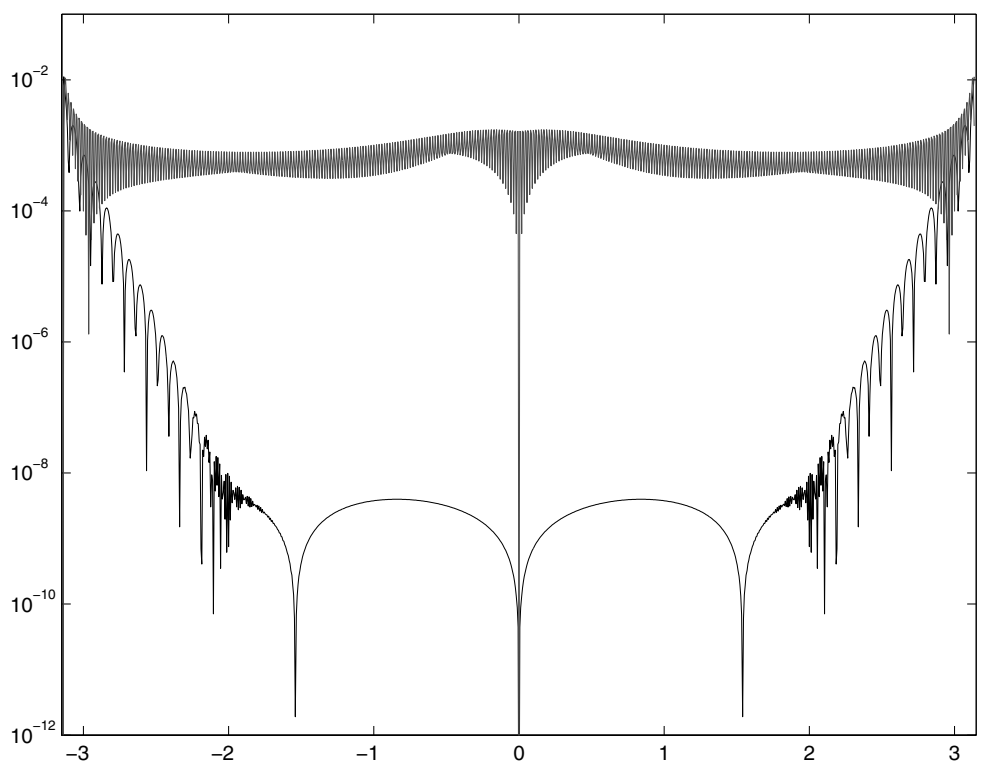

(b)

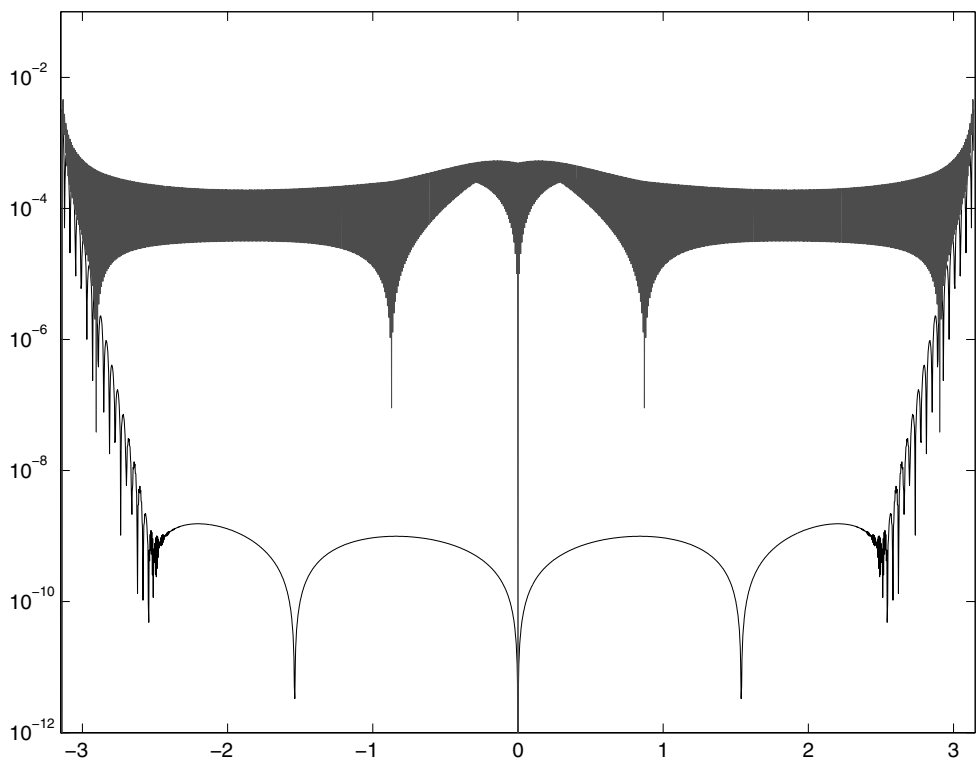

Figure 2.3. The pointwise errors of the two pseudo-spectral methods as a function of time using three different resolutions. The plot is in a log scale. (a) $N=1024$, (b) $N=2048$, both at $t=0.9875$. Initial condition, $u_{0}(x)=\sin (x)$. The error of the $2 / 3$ de-aliasing method (upper curve) is highly oscillatory and spreads out over the entire domain, while the error of the Fourier smoothing method (lower curve) is highly localized near the location of the shock singularity. 
as the vorticity field was mapped onto the Chebyshev mesh was not documented by Kerr (1993). This has led to some ambiguity in reproducing that initial condition which is being resolved by Kerr's group (private communication).

We will summarize the main findings of Hou and Li (2006) in the rest of Section 2. We first illustrate the dynamic evolution of the vortex tubes. In Figure 2.4, we plot the isosurface of the 3D vortex tubes at $t=0$ and $t=6$ respectively. As we can see, the two initial vortex tubes are very smooth and relatively symmetric. Due to the mutual attraction of the two antiparallel vortex tubes, the two vortex tubes approach each other and become flattened dynamically. By time $t=6$, there is already a significant flattening near the centre of the tubes. In Figure 2.5, we plot the local $3 \mathrm{D}$ vortex structure of the upper vortex tube at $t=17$. By this time, the $3 \mathrm{D}$ vortex tube has essentially turned into a thin vortex sheet with rapidly decreasing thickness. The vortex lines become relatively straight. The vortex sheet rolls up near the left edge of the sheet.

In order to see better the dynamic development of the local vortex structure, we plot a sequence of vorticity contours on the symmetry plane at $t=17.5,18,18.5$, and 19, respectively, in Figure 2.6. From these results, we can see that the vortex sheet is compressed in the $z$-direction. It is clear that a thin layer (or a vortex sheet) is formed dynamically. The head of the vortex sheet is a bit thicker than the tail at the beginning. The head of the vortex sheet begins to roll up around $t=16$. By the time $t=19$, the head of the vortex sheet has travelled backward for quite a distance, and the vortex sheet has been compressed quite strongly along the $z$-direction.

We would like to make a few important observations. First of all, the maximum vorticity at a later stage of the computation is actually located near the rolled-up region of the vortex sheet and moves away from the bottom of the vortex sheet. Thus the mechanism of strong compression between the two vortex tubes becomes weaker dynamically at the later time. Secondly, the location of maximum strain and that of maximum vorticity separate as time increases. Thirdly, the relatively 'strong' growth of the maximum velocity between $t=15$ and $t=17$ becomes saturated after $t=17$ when the location of maximum vorticity moves to the rolledup region: see Figure 2.14. All these factors contribute to the dynamic depletion of vortex stretching.

We now perform a convergence study for the two numerical methods using a sequence of resolutions. For the Fourier smoothing method, we use the resolutions $768 \times 512 \times 1536,1024 \times 768 \times 2048$, and $1536 \times 1024 \times$ 3072 respectively. Except for the computation on the largest resolution, $1536 \times 1024 \times 3072$, all computations are carried out from $t=0$ to $t=19$. The computation on the final resolution, $1536 \times 1024 \times 3072$, is started from $t=10$ with the initial condition given by the computation with the 

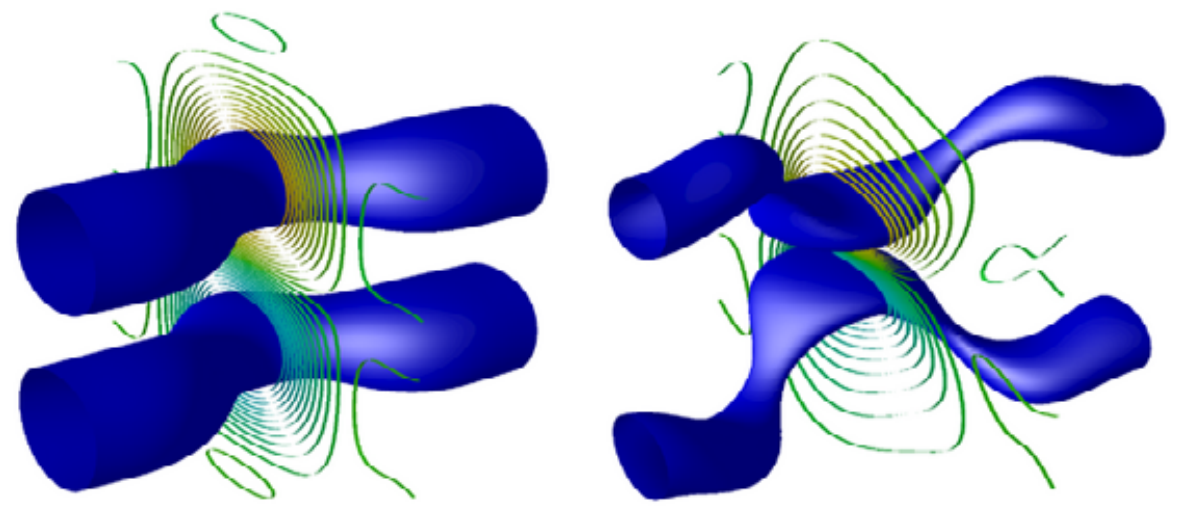

Figure 2.4. The $3 \mathrm{D}$ view of the vortex tube for $t=0$ and $t=6$. The tube is the isosurface at $60 \%$ of the maximum vorticity. The ribbons on the symmetry plane are the contours at other different values.

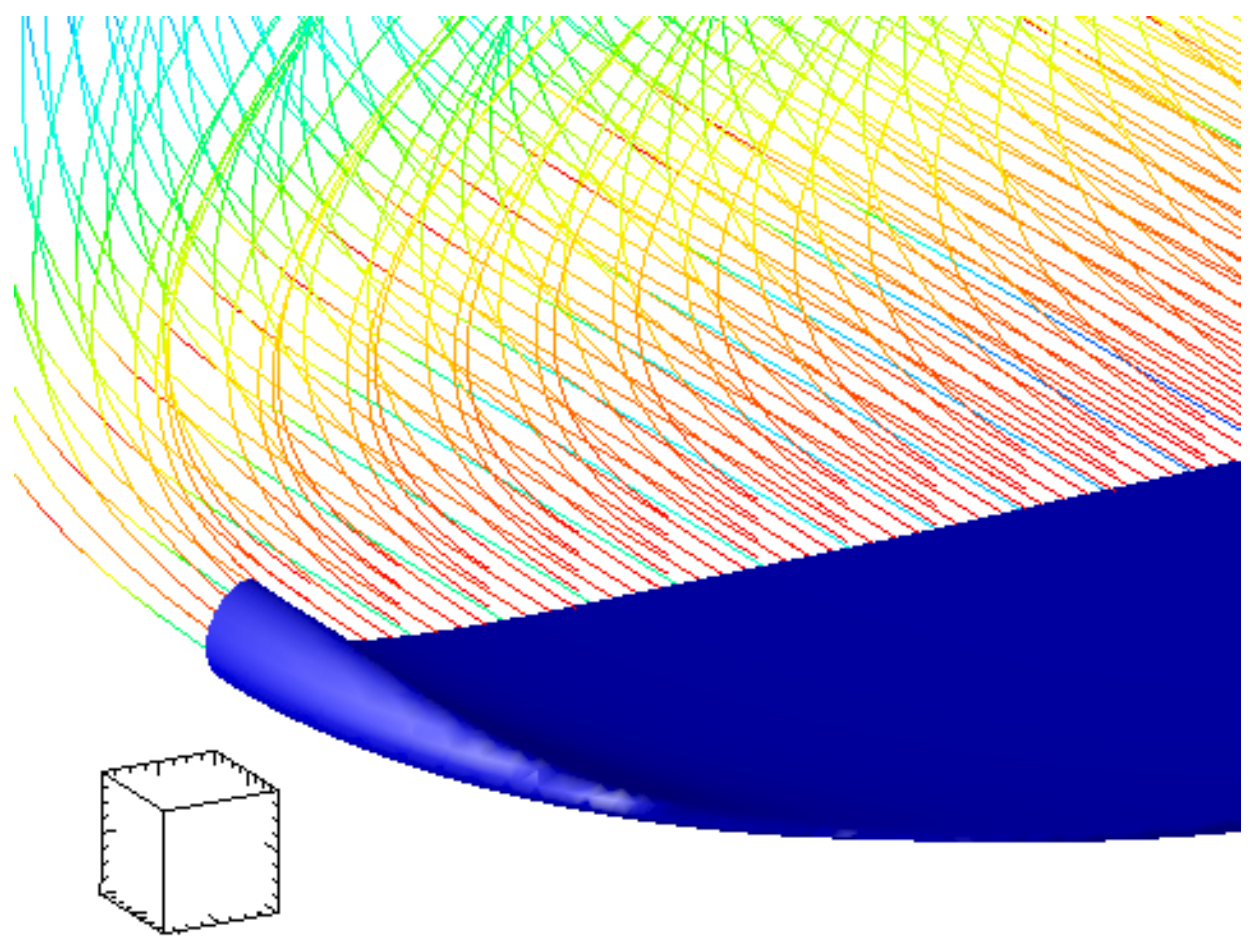

Figure 2.5. The local 3D vortex structures of the upper vortex tube and vortex lines around the maximum vorticity at $t=17$. 

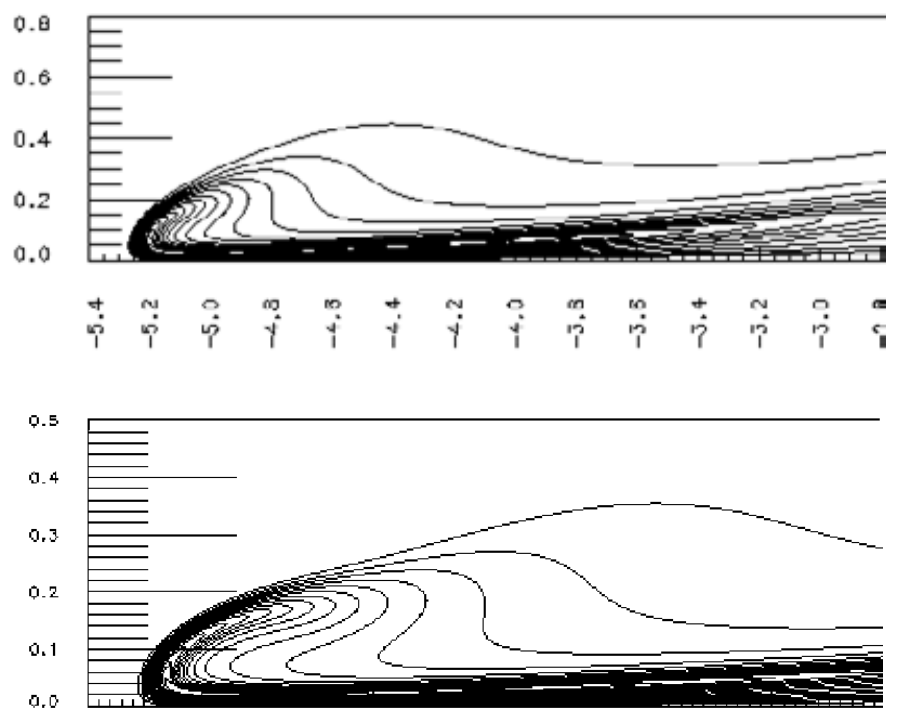

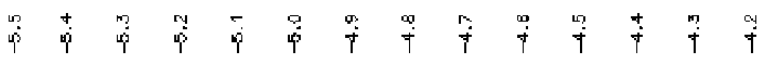
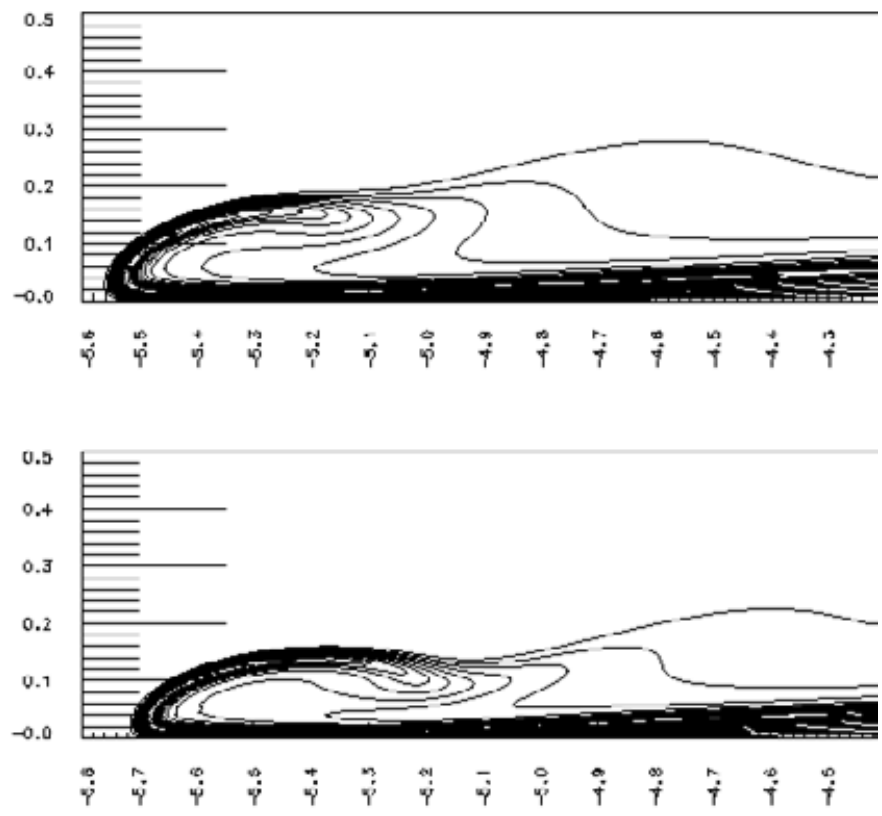

Figure 2.6. The contour of axial vorticity of the upper vortex tube around the maximum vorticity on the symmetry plane (the $x z$-plane) at $t=17.5,18,18.5,19$. 


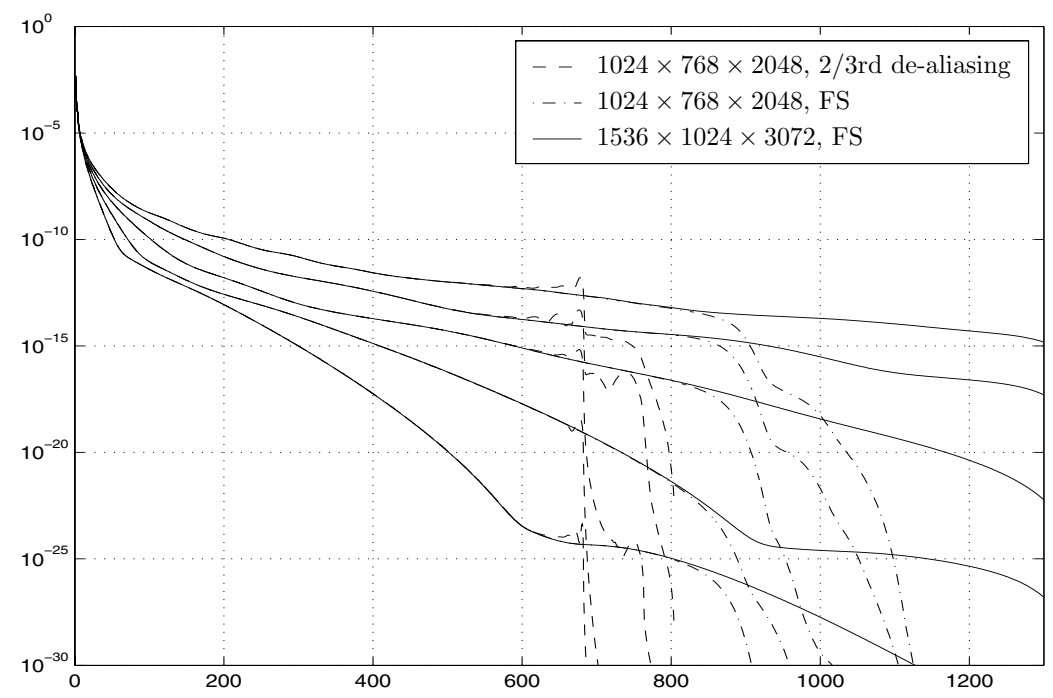

Figure 2.7. The energy spectra versus wavenumbers. The dashed lines and dash-dotted lines are the energy spectra, with resolution $1024 \times 768 \times 2048$, using the $2 / 3$ de-aliasing rule and Fourier smoothing, respectively. The times for the spectra lines are $t=15,16,17,18,19$ respectively.

resolution $1024 \times 768 \times 2048$. For the $2 / 3$ de-aliasing method, we use the resolutions $512 \times 384 \times 1024,768 \times 512 \times 1536$ and $1024 \times 768 \times 2048$ respectively. The computations using these three resolutions are all carried out from $t=0$ to $t=19$. See Hou and $\operatorname{Li}(2006,2007)$ for more details.

In Figure 2.7, we compare the Fourier spectra of the energy obtained by using the $2 / 3$ de-aliasing method with those obtained by the Fourier smoothing method. For a fixed resolution, $1024 \times 768 \times 2048$, we can see that the Fourier spectra obtained by the Fourier smoothing method retain more effective Fourier modes than those obtained by the $2 / 3$ de-aliasing method. This can be seen by comparing the results with the corresponding computations using a higher resolution, $1536 \times 1024 \times 3072$ (the solid lines). Moreover, the Fourier smoothing method does not give the spurious oscillations in the Fourier spectra. In comparison, the Fourier spectra obtained by the $2 / 3$ de-aliasing method produce some spurious oscillations near the $2 / 3$ cut-off point. We would like to emphasize that the Fourier smoothing method conserves the total energy extremely well. More studies including the convergence of the enstrophy spectra can be found in Hou and Li $(2006,2007)$.

It is worth emphasizing that a significant portion of those Fourier modes beyond the $2 / 3$ cut-off position are still accurate for the Fourier smoothing 
method. This portion of the Fourier modes that go beyond the $2 / 3$ cut-off point is about $12 \sim 15 \%$ of total number of modes in each dimension. For $3 \mathrm{D}$ problems, the total number of effective modes in the Fourier smoothing method is about $20 \%$ more than that in the $2 / 3$ de-aliasing method. For our largest resolution, we have about 4.8 billion unknowns. An increase of $20 \%$ in the effective Fourier modes represents a very significant increase in the resolution for a large-scale computation.

\subsection{Comparison of the two spectral methods in physical space}

Next, we compare the solutions obtained by the two methods in physical space for the velocity field and the vorticity. In Figure 2.8, we compare the maximum velocity as a function of time computed by the two methods using resolution $1024 \times 768 \times 2048$. The two solutions are almost indistinguishable. In Figure 2.9, we plot the maximum vorticity as a function of time. The two solutions also agree reasonably well. However, the comparison of the solutions obtained by the two methods at resolutions lower than $1024 \times$ $768 \times 2048$ shows more significant differences between the two methods: see Figure 2.10.

To understand better how the two methods differ in their performance, we examine the contour plots of the axial vorticity in Figures 2.11, 2.12 and 2.13. As we can see, the vorticity computed by the $2 / 3$ de-aliasing method already develops small oscillations at $t=17$. The oscillations grow bigger by $t=18$ (see Figure 2.12), and bigger still at $t=19$ (see Figure 2.13). We note that the oscillations in the axial vorticity contours concentrate near the region where the magnitude of vorticity is close to zero. Thus they have less of an effect on the maximum vorticity. On the other hand, the solution computed by the Fourier smoothing method is still relatively smooth.

\subsection{Dynamic depletion of vortex stretching}

In this section, we present some convincing numerical evidence which shows that there is a strong dynamic depletion of vortex stretching due to local geometric regularity of the vortex lines. We first present the result on the growth of the maximum velocity in time: see Figure 2.14. The growth rate of the maximum velocity plays a critical role in the non-blow-up criteria of Deng, Hou and Yu $(2005,2006 a)$. As we can see from Figure 2.14, the maximum velocity remains bounded up to $t=19$. This is in contrast to the claim in Kerr (2005) that the maximum velocity blows up like $O\left((T-t)^{-1 / 2}\right)$ with $T=18.7$. We note that the velocity field is smoother than the vorticity field. Thus it is easier to resolve the velocity field than the vorticity field. We observe an excellent agreement between the maximum velocity fields computed by the two largest resolutions. Since the velocity field is bounded, the first condition of Theorem 2.1 is satisfied by taking $A=0$. Furthermore, 


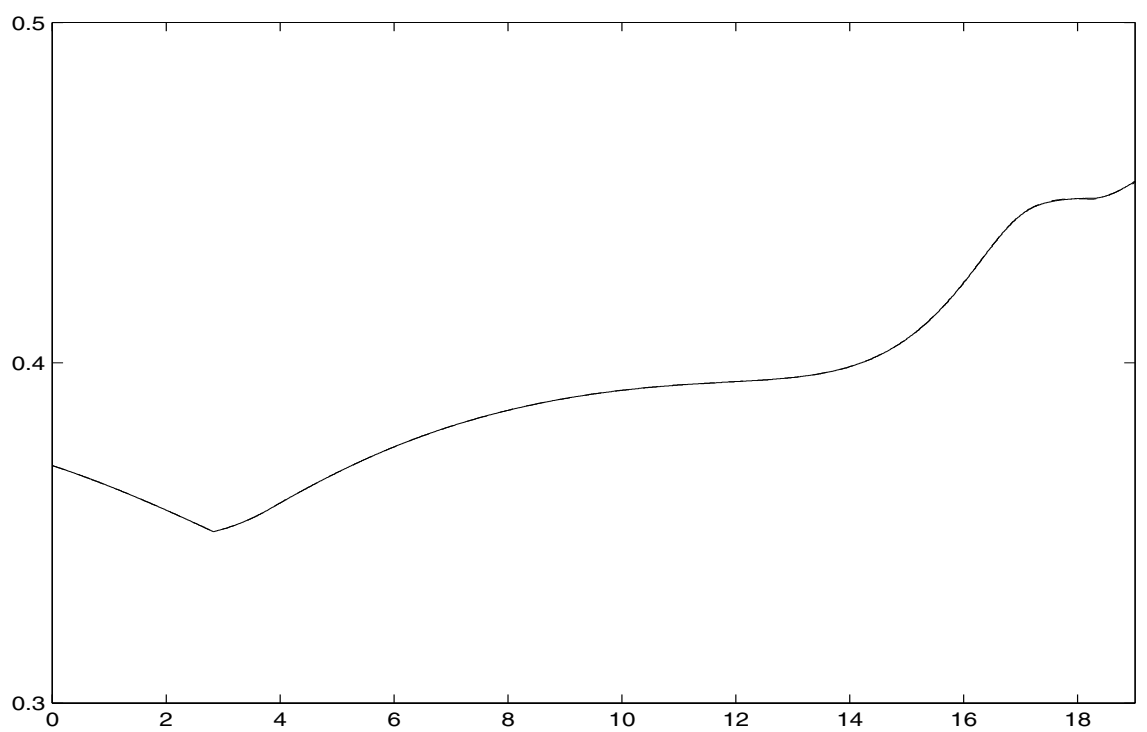

Figure 2.8. Comparison of maximum velocity as a function of time computed by two methods. Solid line, solution obtained by the Fourier smoothing method; dashed line, solution obtained by the $2 / 3$ de-aliasing method. Resolution $1024 \times 768 \times 2048$ for both methods.

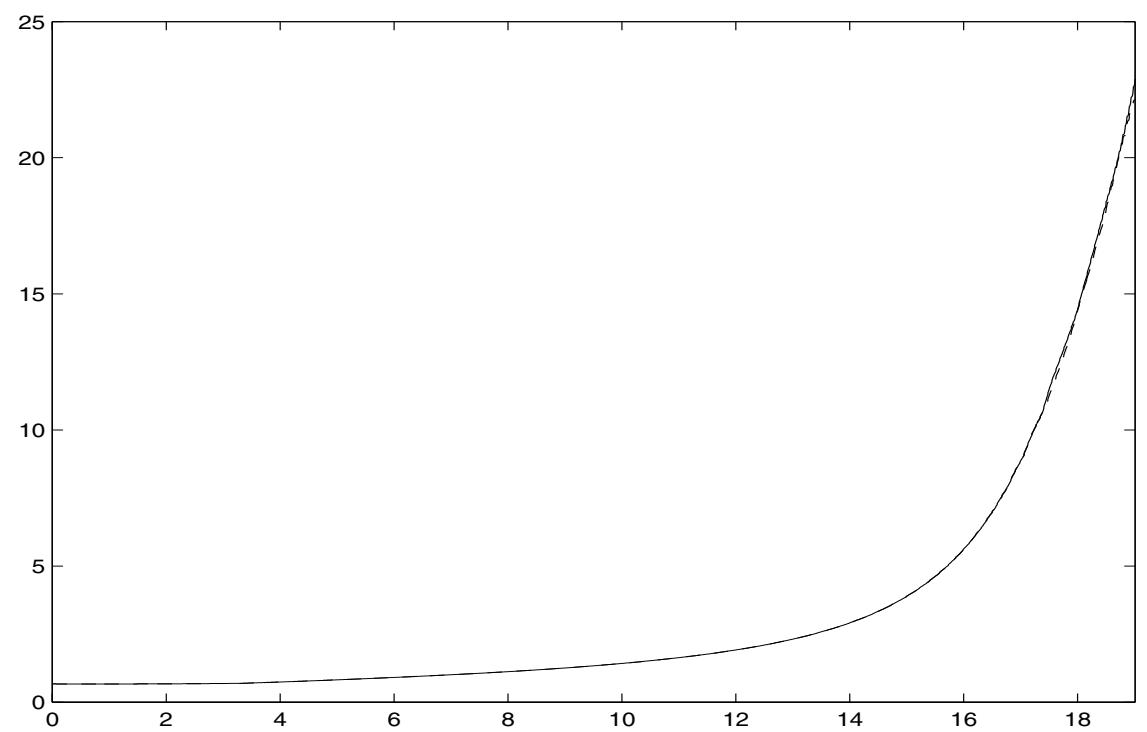

Figure 2.9. Comparison of maximum vorticity as a function of time computed by two methods. Solid line, solution obtained by the Fourier smoothing method; dashed line, solution obtained by the $2 / 3$ de-aliasing method. Resolution $1024 \times 768 \times 2048$ for both methods. 


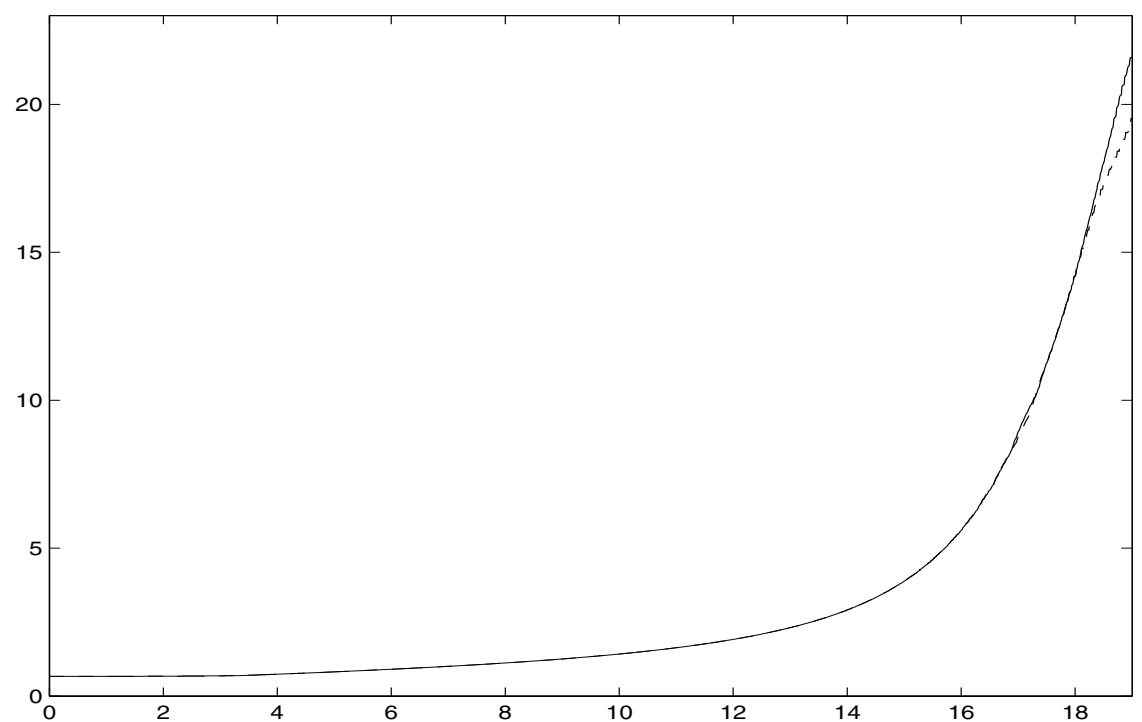

Figure 2.10. Comparison of maximum vorticity as a function of time computed by two methods. Solid line, solution obtained by the Fourier smoothing method; dashed line, solution obtained by the $2 / 3$ de-aliasing method. Resolution $768 \times 512 \times 1024$ for both methods.

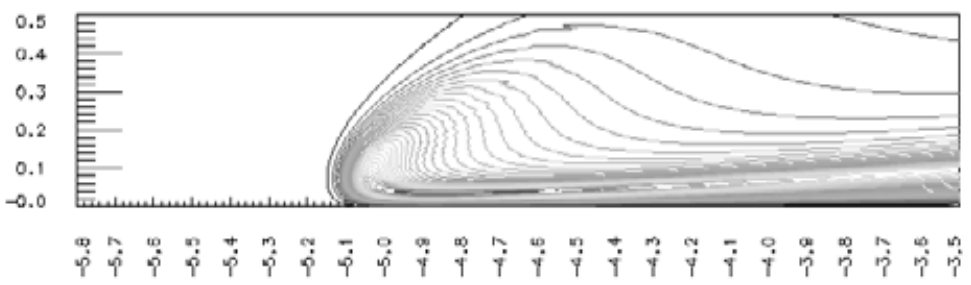

(a)

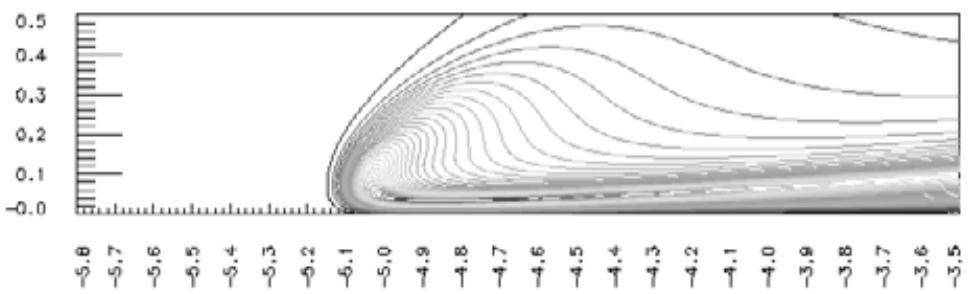

(b)

Figure 2.11. Comparison of axial vorticity contours at $t=17$ computed by two methods. (a) Solution obtained by the $2 / 3$ de-aliasing method, (b) solution obtained by the Fourier smoothing method. Resolution $1024 \times 768 \times 2048$ for both methods. 


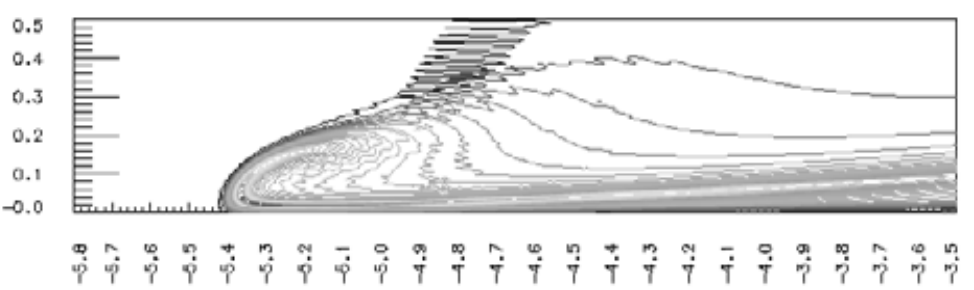

(a)

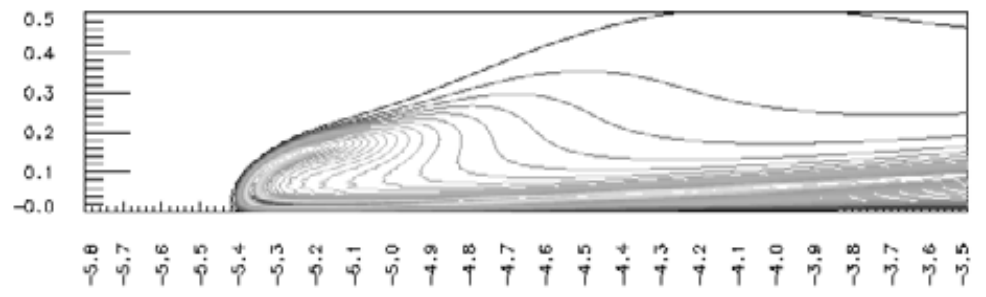

(b)

Figure 2.12. Comparison of axial vorticity contours at $t=18$ computed by two methods. (a) Solution obtained by the $2 / 3$ de-aliasing method, (b) solution obtained by the Fourier smoothing method. Resolution $1024 \times 768 \times 2048$ for both methods.

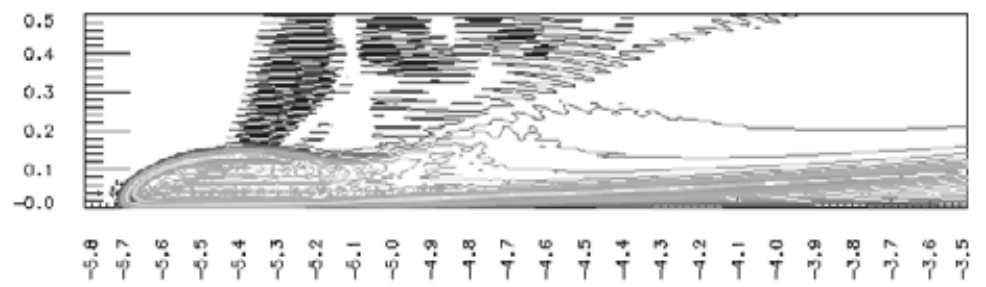

(a)

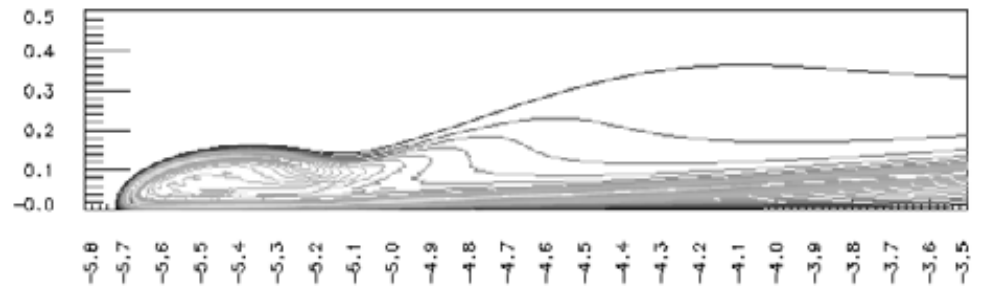

(b)

Figure 2.13. Comparison of axial vorticity contours at $t=19$ computed by two methods. (a) Solution obtained by the $2 / 3$ de-aliasing method, (b) solution obtained by the Fourier smoothing method. Resolution $1024 \times 768 \times 2048$ for both methods. 
since both $\nabla \cdot \boldsymbol{\xi}$ and $\kappa$ are bounded by $O\left((T-t)^{-1 / 2}\right)$ in the inner region of size $(T-t)^{1 / 2} \times(T-t)^{1 / 2} \times(T-t)$ (Kerr 2005), the second condition of Theorem 2.1 is satisfied with $B=1 / 2$ by taking a segment of the vortex line with length $(T-t)^{1 / 2}$ within this inner region. Thus Theorem 2.1 can be applied to our computation, which implies that the solution of the 3D Euler equations remains smooth at least up to $T=19$.

We also study the maximum vorticity as a function of time. The maximum vorticity is found to increase rapidly from the initial value of 0.669 to 23.46 at the final time $t=19$, a factor of 35 increase from its initial value. Our computations show no sign of finite-time blow-up of the 3D Euler equations up to $T=19$, beyond the singularity time predicted by Kerr. The maximum vorticity computed by resolution $1024 \times 768 \times 2048$ agrees very well with that computed by resolution $1536 \times 1024 \times 3072$ up to $t=17.5$. There is some mild disagreement towards the end of the computation. This indicates that a very high space resolution is needed to capture the rapid growth of maximum vorticity at the final stage of the computation.

In order to understand the nature of the dynamic growth in vorticity, we examine the degree of nonlinearity in the vortex stretching term. In Figure 2.15 , we plot the quantity, $\|\xi \cdot \nabla \mathbf{u} \cdot \boldsymbol{\omega}\|_{\infty}$, as a function of time. If the maximum vorticity indeed blew up like $O\left((T-t)^{-1}\right)$, as alleged in Kerr (1993), this quantity should have been quadratic as a function of maximum vorticity. We find that there is tremendous cancellation in this vortex stretching term. It actually grows more slowly than $C\|\vec{\omega}\|_{\infty} \log \left(\|\vec{\omega}\|_{\infty}\right)$ : see Figure 2.15. It is easy to show that $\|\xi \cdot \nabla \mathbf{u} \cdot \boldsymbol{\omega}\|_{\infty} \leq C\|\vec{\omega}\|_{\infty} \log \left(\|\vec{\omega}\|_{\infty}\right)$ would imply at most doubly exponential growth in the maximum vorticity. Indeed, as demonstrated by Figure 2.16, the maximum vorticity does not grow more rapidly than doubly exponential in time. We have also generated a similar plot by extracting the data from Kerr (1993). We find that $\log \left(\log \left(\|\boldsymbol{\omega}\|_{\infty}\right)\right)$ basically scales linearly with respect to $t$ from $14 \leq t \leq 17.5$ when Kerr's computations are still reasonably resolved. This implies that the maximum vorticity up to $t=17.5$ in his computations does not grow more rapidly than doubly exponential in time. This is consistent with our conclusion.

We study the decay rate in the energy spectrum in Figure 2.17 at $t=$ $16,17,18,19$. A finite-time blow-up of enstrophy would imply that the energy spectrum decays no more rapidly than $|k|^{-3}$. Our computations show that the energy spectrum approaches $|k|^{-3}$ for $|k| \leq 100$ as time increases to $t=19$. This is in qualitative agreement with Kerr's results. Note that there are fewer than 100 modes available along the $\left|k_{x}\right|$ - or $\left|k_{y}\right|$-direction in Kerr's computations: see Figure 18(a),(b) of Kerr (1993). On the other hand, our computations show that the high-frequency Fourier spectrum for $100 \leq|k| \leq 1300$ decays much more rapidly than $|k|^{-3}$, as one can see from Figure 2.17. This indicates that there is no blow-up in enstrophy. 


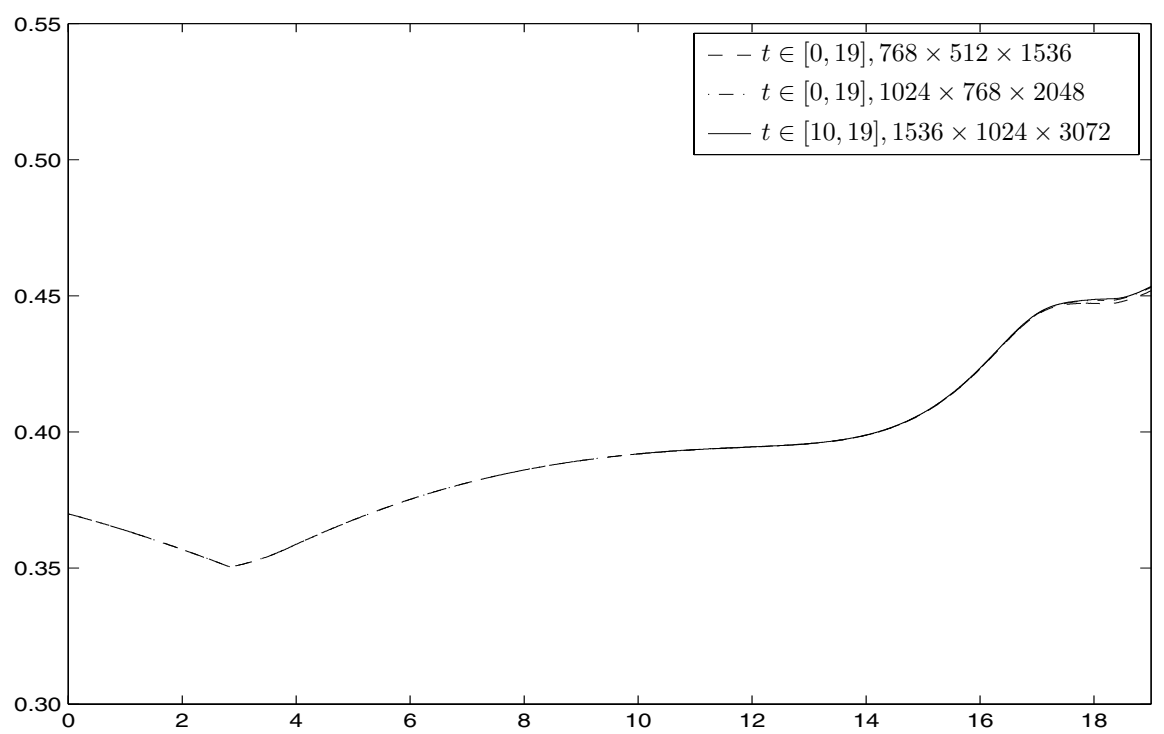

Figure 2.14. Maximum velocity $\|\mathbf{u}\|_{\infty}$ in time using three different resolutions.

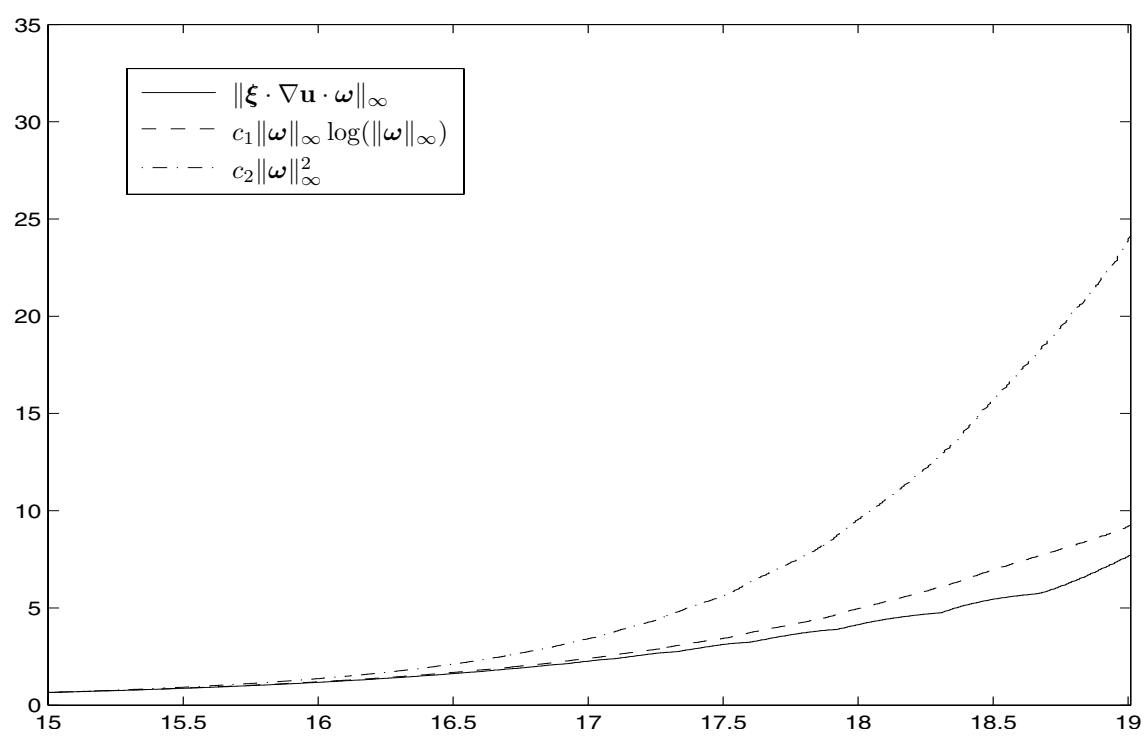

Figure 2.15. Study of the vortex stretching term in time, resolution $1536 \times 1024 \times 3072$. The inequality $|\boldsymbol{\xi} \cdot \nabla \mathbf{u} \cdot \boldsymbol{\omega}| \leq c_{1}|\boldsymbol{\omega}| \log |\boldsymbol{\omega}|$ and $\frac{D}{D t}|\boldsymbol{\omega}|=\boldsymbol{\xi} \cdot \nabla \mathbf{u} \cdot \boldsymbol{\omega}$ implies $|\boldsymbol{\omega}|$ bounded by a double exponential. 


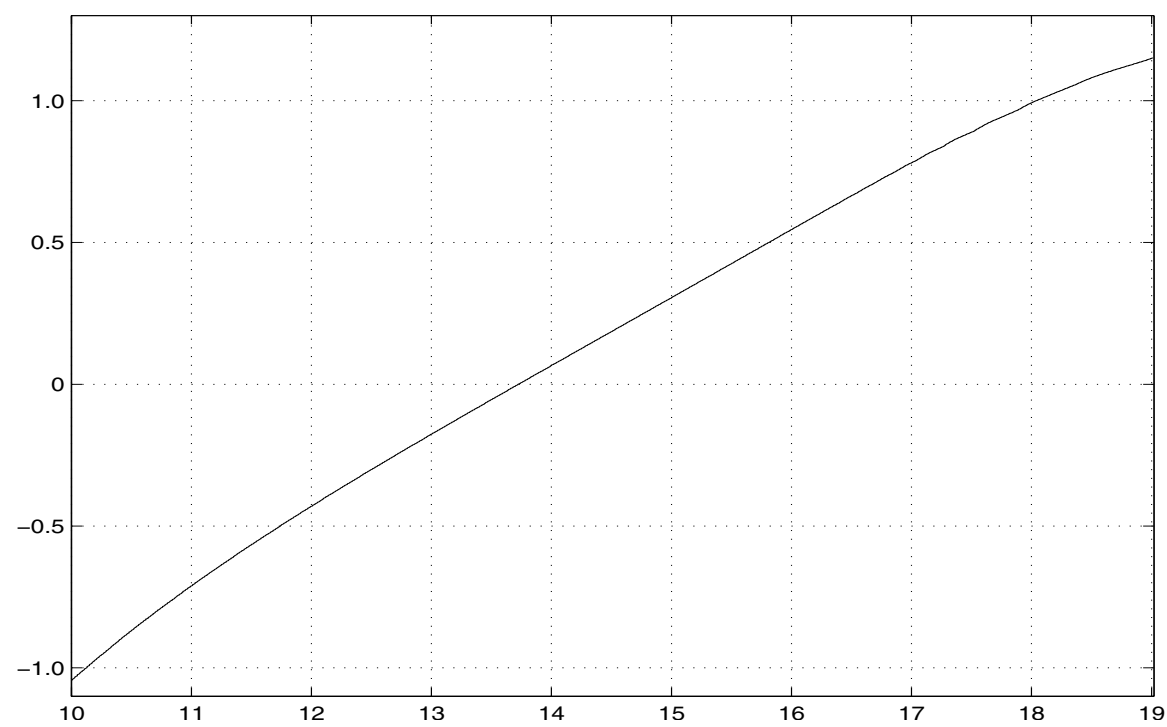

Figure 2.16. The plot of $\log \log \|\boldsymbol{\omega}\|_{\infty}$ versus time, resolution $1536 \times 1024 \times 3072$.

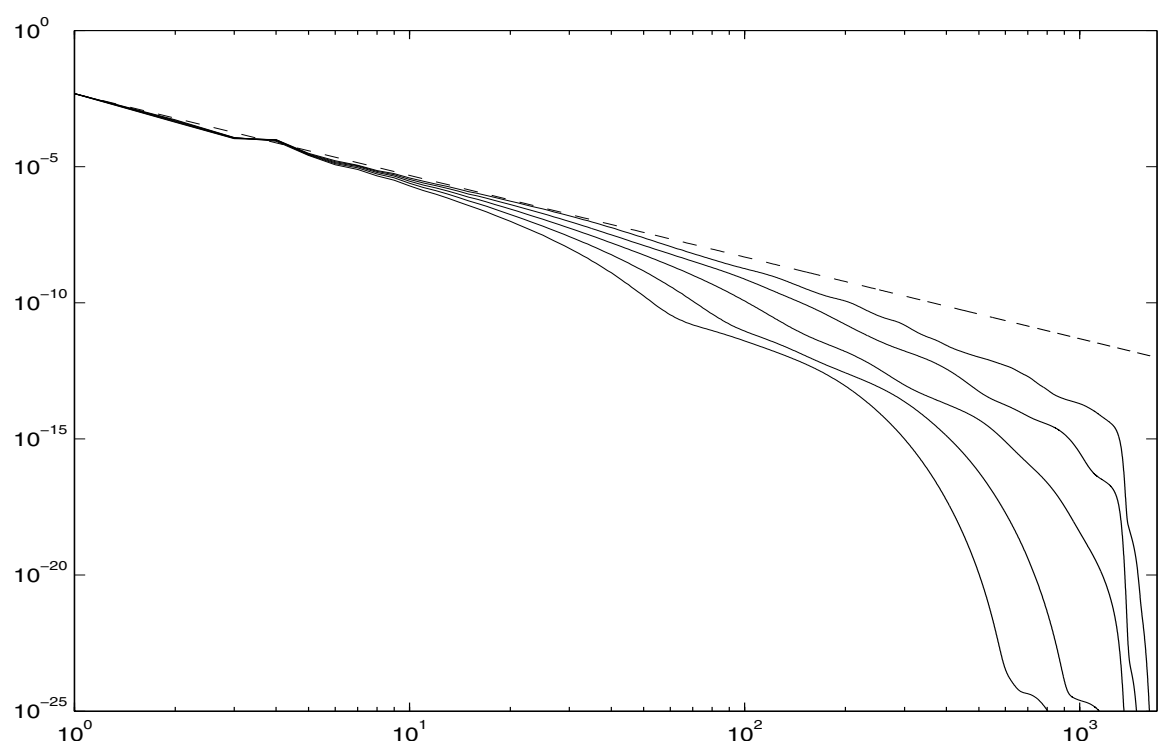

Figure 2.17. The energy spectra for velocity at $t=15,16,17,18,19$ (from bottom to top) in log-log scale. Dashed line, $k^{-3}$. 
Table 2.1. The alignment of the vorticity vector and the eigenvectors of $S$ around the point of maximum vorticity with resolution $1536 \times 1024 \times 3072$. Here, $\theta_{i}$ is the angle between the $i$ th eigenvector of $S$ and the vorticity vector.

\begin{tabular}{cr|cc|cc|cc}
\hline \hline Time & $|\boldsymbol{\omega}|$ & $\lambda_{1}$ & $\theta_{1}$ & $\lambda_{2}$ & $\theta_{2}$ & $\lambda_{3}$ & $\theta_{3}$ \\
\hline 16.012 & 5.628 & -1.508 & 89.992 & 0.206 & 0.007 & 1.302 & 89.998 \\
16.515 & 7.016 & -1.864 & 89.995 & 0.232 & 0.010 & 1.631 & 89.990 \\
17.013 & 8.910 & -2.322 & 89.998 & 0.254 & 0.006 & 2.066 & 89.993 \\
17.515 & 11.430 & -2.630 & 89.969 & 0.224 & 0.085 & 2.415 & 89.920 \\
18.011 & 14.890 & -3.625 & 89.969 & 0.257 & 0.036 & 3.378 & 89.979 \\
18.516 & 19.130 & -4.501 & 89.966 & 0.246 & 0.036 & 4.274 & 89.984 \\
19.014 & 23.590 & -5.477 & 89.966 & 0.247 & 0.034 & 5.258 & 89.994 \\
\hline
\end{tabular}

It is interesting to ask how the vorticity vector aligns with the eigenvectors of the deformation tensor. Recall that the vorticity equations can be written as

$$
\frac{\partial}{\partial t} \boldsymbol{\omega}+(\mathbf{u} \cdot \nabla) \boldsymbol{\omega}=S \cdot \boldsymbol{\omega}, \quad S=\frac{1}{2}\left(\nabla \mathbf{u}+\nabla^{T} \mathbf{u}\right)
$$

(see Majda and Bertozzi (2002)). Let $\lambda_{1}<\lambda_{2}<\lambda_{3}$ be the three eigenvalues of $S$. The incompressibility condition implies that $\lambda_{1}+\lambda_{2}+\lambda_{3}=0$. If the vorticity vector aligns with the eigenvector corresponding to $\lambda_{3}$, which gives the maximum rate of stretching, then it is very likely that the 3D Euler equations would blow up in a finite time.

In Table 2.1, we document the alignment information of the vorticity vector around the point of maximum vorticity with resolution $1536 \times 1024 \times$ 3072. In this table, $\theta_{i}$ is the angle between the $i$ th eigenvector of $S$ and the vorticity vector. One can see clearly that for $16 \leq t \leq 19$ the vorticity vector at the point of maximum vorticity is almost perfectly aligned with the second eigenvector of $S$. Note that the second eigenvalue, $\lambda_{2}$, is about 20 times smaller in magnitude than the largest eigenvalue $\lambda_{3}$, and does not grow much in time. The alignment of the vorticity vector with the second eigenvector of the deformation tensor is another indication that there is a strong dynamic depletion of vortex stretching.

\subsection{Global regularity of large anisotropic initial data}

The numerical studies of the 3D Euler equations by Hou and Li (2006) strongly suggest that the support of maximum vorticity becomes severely flattened and develops an anisotropic scaling as vorticity increases rapidly in time. This seems quite generic and is a consequence of the incompressibility 
and the Lagrangian structure of the vorticity equation. Convection plays an essential role in producing this anisotropic structure of the solution. Motivated by the desire to understand how the local anisotropic structure of the solution near the support of maximum vorticity may lead to the depletion of vortex stretching, Hou, Lei and Li (2008) recently studied the 3 D axisymmetric Navier-Stokes equations with large anisotropic data. They proved the global regularity of the 3D Navier-Stokes equations for a family of large anisotropic initial data. Moreover, they obtained a global bound of the solution in terms of its initial data in some $L^{p}$-norm. Their results also revealed some interesting dynamic growth behaviour of the solution due to the interaction between the axial vorticity and the the derivative of vorticity.

Specifically, let $u^{\theta}$ and $\omega^{\theta}$ be the angular velocity and vorticity components of the 3D axisymmetric Navier-Stokes equations. They considered initial data for $u^{\theta}$ and $\omega^{\theta}$ that have the following scaling property:

$$
u^{\theta}(r, z, 0)=\frac{1}{\epsilon^{1-\delta}} U_{0}(\epsilon r, z), \quad \omega^{\theta}(r, z, 0)=\frac{1}{\epsilon^{1-\delta}} W_{0}(\epsilon r, z),
$$

where $r=\sqrt{x^{2}+y^{2}}, \delta$ and $\epsilon$ are some small positive parameters, and the rescaled profiles $U_{0} / r$ and $W_{0} / r$ are bounded in $L^{2 p}$ and $L^{2 q}$, respectively, for some $p$ and $q$ with $p=2 q$; note that $u^{\theta}$ and $\omega^{\theta}$ must satisfy a compatibility condition: $\left.u^{\theta}\right|_{r=0}=0=\left.\omega^{\theta}\right|_{r=0}$ (Liu and Wang 2006). We remark that these initial data are not small. In fact, we have

$$
\left\|\mathbf{u}_{0}\right\|_{L^{2}\left(\mathbb{R}^{2} \times[0,1]\right)}\left\|\nabla \mathbf{u}_{0}\right\|_{L^{2}\left(\mathbb{R}^{2} \times[0,1]\right)}=\frac{C_{0}}{\epsilon^{4-2 \delta}} \gg 1,
$$

for $\epsilon$ small, where $\mathbf{u}_{0}$ is the initial velocity vector. Thus the classical regularity analysis for small initial data does not apply to these sets of anisotropic initial data.

Hou, Lei and Li (2008) proved the global regularity of the 3D axisymmetric Navier-Stokes equations for initial data (2.10) by exploring the anisotropic structure of the solution for $\epsilon$ small. They also obtained a global bound on $\left\|u^{\theta} / r\right\|_{L^{2 p}}$ and $\left\|\omega^{\theta} / r\right\|_{L^{2 q}}$ in terms of their initial data. Note that by using the scaling invariance property of the Navier-Stokes equations, their global regularity result also applies to the following rescaled initial data:

$$
u^{\theta}(r, z, 0)=\frac{1}{\epsilon^{2-\delta}} U_{0}\left(r, \frac{z}{\epsilon}\right), \quad \omega^{\theta}(r, z, 0)=\frac{1}{\epsilon^{3-\delta}} W_{0}\left(r, \frac{z}{\epsilon}\right),
$$

and

$$
u^{\theta}(r, z, 0)=\frac{1}{\epsilon} U_{0}\left(\frac{r}{\epsilon^{1-\delta}}, \frac{z}{\epsilon}\right), \quad \omega^{\theta}(r, z, 0)=\frac{1}{\epsilon^{2}} W_{0}\left(\frac{r}{\epsilon^{1-\delta}}, \frac{z}{\epsilon}\right) .
$$

Note that the parameters $\epsilon$ in the initial data (2.10)-(2.11) and $\delta$ in (2.12) measure the degree of anisotropy of the initial data. If $\delta=0$, then the 
initial data (2.12) become isotropic, i.e.,

$$
\mathbf{u}_{0}(x, y, z)=\frac{1}{\epsilon} \mathbf{U}_{0}\left(\frac{x}{\epsilon}, \frac{y}{\epsilon}, \frac{z}{\epsilon}\right) .
$$

Their analysis would break down when there is no anisotropic scaling in the initial data, i.e., $\delta=0$. Clearly, if the analysis could be extended to the case of $\delta=0$, one would prove the global regularity of the 3D axisymmetric Navier-Stokes equations for general initial data by using the scaling invariance property of the Navier-Stokes equations. It is interesting to note that by using an anisotropic scaling of the initial data, we turn the global regularity of the 3D Navier-Stokes equations into a critical case of $\delta=0$.

We remark that the global regularity results of Hou, Lei and Li (2008) were obtained on a regular size domain, $\mathbb{R}^{2} \times[0,1]$, for initial data (2.10). In this sense, their results are different from those global regularity results obtained for a thin domain, $\Omega_{\epsilon}=Q_{1} \times[0, \epsilon]$ with $Q_{1}$ being a bounded domain in $\mathbb{R}^{2}$. The global regularity of the $3 \mathrm{D}$ Navier-Stokes equations in a thin domain of the form $\Omega_{\epsilon}$ has been studied by Raugel and Sell in a series of papers (Raugel and Sell 1993a, 1994, 1993b). They proved the global regularity of the 3D Navier-Stokes equations under the assumption that $\left\|\nabla \mathbf{u}_{0}\right\|_{L^{2}\left(\Omega_{\epsilon}\right)}^{2} \leq C_{0} \ln \frac{1}{\epsilon}$. This is an improvement over the classical global regularity result for small data, which requires $\left\|\nabla \mathbf{u}_{0}\right\|_{L^{2}\left(\Omega_{\epsilon}\right)}^{2} \leq C^{*} \epsilon$ (Raugel and Sell 1993a). One may interpret the global regularity result of Hou, Lei and Li with initial data $(2.11)$ as a result on a generalized thin domain. Note that the initial data given by (2.11) satisfy the following bound: $\left\|\nabla \mathbf{u}_{0}\right\|_{L^{2}\left(\Omega_{\epsilon}\right)}^{2}=C_{0} \epsilon^{-5+2 \delta}$ (here $\delta>0$ can be made arbitrarily small), which is much larger than the corresponding bound $C_{0} \ln \frac{1}{\epsilon}$ required by the global regularity analysis of Raugel and Sell $(1993 a, 1994,1993 b)$.

\section{Dynamic stability of 3D Navier-Stokes equations}

The axisymmetric 3D Navier-Stokes equation with swirl is perhaps the simplest form of the 3D Navier-Stokes equations, yet still retains the most essential difficulties of the 3D Navier-Stokes equations. It has attracted a lot of attention in recent years. Although some partial progress has been made in studying the global regularity of the axisymmetric Navier-Stokes equations with swirl using energy estimates (see, e.g., Chae and Lee (2002) and references cited there), the question of global regularity for general initial data is still an open question.

Hou and $\mathrm{Li}$ (2008a) studied the dynamic stability of the axisymmetric Navier-Stokes equations with swirl via a new 1D model. This model is derived from the axisymmetric Navier-Stokes equations along the symmetry axis. Surprisingly, this model is an exact reduction of the $3 \mathrm{D}$ axisymmetric 
Navier-Stokes equations along the symmetry axis. It captures the essential nonlinear features of the 3D Navier-Stokes equations. One of the important findings by Hou and $\mathrm{Li}(2008 a)$ was that the convection term plays an essential role in cancelling some of the vortex stretching terms. Specifically, they found a positive Lyapunov function which satisfies a new conservation law and a maximum principle. This holds for both the viscous and inviscid cases. This a priori pointwise estimate plays a critical role in obtaining nonlinear stability and global regularity of the 1D model. Using this a priori estimate, they proved global regularity of the 3D Navier-Stokes equations for a family of large data, which can experience large transient dynamic growth but remain smooth for all times.

It is worth emphasizing that such subtle dynamic stability properties of the 3D Navier-Stokes equations would have been completely missed by using the traditional energy estimates. Traditional energy estimates are too crude to capture some of the most essential properties of the 3D incompressible Navier-Stokes equations. To illustrate its limitations, we briefly review how the energy estimates are used in proving global regularity of the 3D NavierStokes equations.

For incompressible Navier-Stokes equations, one of the most important a priori estimates is the energy identity. More precisely, for any strong solution $\mathbf{u}$, we have

$$
\frac{1}{2} \frac{\mathrm{d}}{\mathrm{d} t} \int|\mathbf{u}|^{2} \mathrm{~d} \mathbf{x}+\nu \int|\nabla \mathbf{u}|^{2} \mathrm{~d} \mathbf{x}=0,
$$

by observing $\int \mathbf{u} \cdot(\mathbf{u} \cdot \nabla \mathbf{u}) \mathrm{d} \mathbf{x}=-\frac{1}{2} \int(\nabla \cdot \mathbf{u})|\mathbf{u}|^{2} \mathrm{~d} \mathbf{x}=0$, since $\mathbf{u}$ is divergencefree. Unfortunately, this energy identity is not strong enough to rule out finite-time singularities. To prove global regularity, we need to obtain control in a stronger norm, either in $\|\mathbf{u}\|_{L^{p}}$ with $p \geq 3$ or in $\|\boldsymbol{\omega}\|_{L^{2}}$. To illustrate the main difficulty of the traditional energy estimates, let us perform energy estimates for the vorticity equation:

$$
\frac{1}{2} \frac{\mathrm{d}}{\mathrm{d} t} \int|\boldsymbol{\omega}|^{2} \mathrm{~d} \mathbf{x}+\nu \int|\nabla \boldsymbol{\omega}|^{2} \mathrm{~d} \mathbf{x}=\int \boldsymbol{\omega} \cdot \nabla \mathbf{u} \cdot \boldsymbol{\omega} \mathrm{d} \mathbf{x} .
$$

Again, the convection term does not contribute to the $L^{2}$-norm of vorticity (or any $L^{p}$-norm with $p>1$ ). The main difficulty is to control the vortex stretching term. Using the Sobolev embedding theory, one can show that

$$
\int \boldsymbol{\omega} \cdot \nabla \mathbf{u} \cdot \boldsymbol{\omega} \mathrm{d} \mathbf{x} \leq C_{\nu}\left(\int|\boldsymbol{\omega}|^{2} \mathrm{~d} \mathbf{x}\right)^{3}+\frac{\nu}{2} \int|\nabla \boldsymbol{\omega}|^{2} \mathrm{~d} \mathbf{x},
$$

which can not be improved. This implies that

$$
\frac{1}{2} \frac{\mathrm{d}}{\mathrm{d} t} \int|\boldsymbol{\omega}|^{2} \mathrm{~d} \mathbf{x}+\frac{\nu}{2} \int|\nabla \boldsymbol{\omega}|^{2} \mathrm{~d} \mathbf{x} \leq C_{\nu}\left(\int|\boldsymbol{\omega}|^{2} \mathrm{~d} \mathbf{x}\right)^{3}
$$


Unfortunately, the above estimate does not imply global regularity for large data even if we use the energy identity (3.1). However, the estimate (3.4) can be used to obtain global regularity for small initial data. To see this, we substitute the following interpolation inequality,

$$
\left(\int|\boldsymbol{\omega}|^{2} \mathrm{~d} \mathbf{x}\right)^{2}=\|\boldsymbol{\omega}\|_{L^{2}}^{4} \leq C_{0}\|\mathbf{u}\|_{L^{2}}^{2}\|\nabla \boldsymbol{\omega}\|_{L^{2}}^{2}
$$

into (3.4) to obtain

$$
\frac{1}{2} \frac{\mathrm{d}}{\mathrm{d} t}\|\boldsymbol{\omega}\|_{L^{2}}^{2} \leq\left(C_{\nu} C_{0}\|\mathbf{u}\|_{L^{2}}^{2}\|\boldsymbol{\omega}\|_{L^{2}}^{2}-\frac{\nu}{2}\right)\|\nabla \boldsymbol{\omega}\|_{L^{2}}^{2} \leq 0,
$$

provided that

$$
C_{\nu} C_{0}\left\|\mathbf{u}_{0}\right\|_{L^{2}}^{2}\left\|\boldsymbol{\omega}_{0}\right\|_{L^{2}}^{2} \leq \frac{\nu}{2} .
$$

Since $\|\mathbf{u}(t)\|_{L^{2}}^{2} \leq\left\|\mathbf{u}_{0}\right\|_{L^{2}}^{2}$ for all $t$, condition (3.7) and inequality (3.6) imply that $\|\boldsymbol{\omega}(t)\|_{L^{2}}^{2} \leq\left\|\boldsymbol{\omega}_{0}\right\|_{L^{2}}^{2}$ for all times. Note that $\left\|\boldsymbol{\omega}_{0}\right\|_{L^{2}}^{2}=\left\|\nabla \mathbf{u}_{0}\right\|_{L^{2}}^{2}$. Thus we can also replace (3.7) by

$$
C_{\nu} C_{0}\left\|\mathbf{u}_{0}\right\|_{L^{2}}^{2}\left\|\nabla \mathbf{u}_{0}\right\|_{L^{2}}^{2} \leq \frac{\nu}{2}
$$

Due to the incompressibility condition, convection plays no role in the energy estimate. The same estimate can be also applied to the following nonlinear diffusion equation:

$$
w_{t}=w^{2}+\nu \Delta w
$$

An energy estimate gives

$$
\frac{1}{2} \frac{\mathrm{d}}{\mathrm{d} t} \int|w|^{2} \mathrm{~d} \mathbf{x}+\nu \int|\nabla w|^{2} \mathrm{~d} \mathbf{x}=\int w^{3} \mathrm{~d} \mathbf{x} .
$$

Using an embedding inequality similar to (3.3), we get

$$
\frac{1}{2} \frac{\mathrm{d}}{\mathrm{d} t} \int|w|^{2} \mathrm{~d} \mathbf{x}+\frac{\nu}{2} \int|\nabla w|^{2} \mathrm{~d} \mathbf{x} \leq C_{\nu}\left(\int|w|^{2} \mathrm{~d} \mathbf{x}\right)^{3},
$$

which is identical to (3.4).

However, it is well known that (3.9) can develop a finite-time isotropic self-similar blow-up solution, which does not violate the energy identity (3.1), in the sense that $\int_{0}^{T}\|w(t)\|_{L^{2}}^{2} \mathrm{~d} t<\infty$. The above analysis shows that energy estimates can not distinguish a nonlinear diffusion equation, which has a finite-time blow-up solution, from the 3D Navier-Stokes equations, which have completely different physical properties and may not necessarily blow up in finite time. 


\subsection{Reformulation of $3 D$ axisymmetric Navier-Stokes equations}

Consider the 3D axisymmetric incompressible Navier-Stokes equations

$$
\begin{aligned}
u_{t}^{\theta}+u^{r} u_{r}^{\theta}+u^{z} u_{z}^{\theta} & =\nu\left(\nabla^{2}-\frac{1}{r^{2}}\right) u^{\theta}-\frac{1}{r} u^{r} u^{\theta}, \\
\omega_{t}^{\theta}+u^{r} \omega_{r}^{\theta}+u^{z} \omega_{z}^{\theta} & =\nu\left(\nabla^{2}-\frac{1}{r^{2}}\right) \omega^{\theta}+\frac{1}{r}\left(\left(u^{\theta}\right)^{2}\right)_{z}+\frac{1}{r} u^{r} \omega^{\theta}, \\
-\left(\nabla^{2}-\frac{1}{r^{2}}\right) \psi^{\theta} & =\omega^{\theta},
\end{aligned}
$$

where $r=\sqrt{x^{2}+y^{2}}, u^{\theta}, \omega^{\theta}$ and $\psi^{\theta}$ are the angular components of the velocity, vorticity and stream function respectively, and

$$
u^{r}=-\left(\psi^{\theta}\right)_{z} \quad u^{z}=\frac{1}{r}\left(r \psi^{\theta}\right)_{r} .
$$

Note that equations (3.12)-(3.14) completely determine the evolution of the $3 \mathrm{D}$ axisymmetric Navier-Stokes equations.

Hou and Li (2008a) introduced the following new variables,

$$
u_{1}=u^{\theta} / r, \quad \omega_{1}=\omega^{\theta} / r, \quad \psi_{1}=\psi^{\theta} / r,
$$

and derived the following equivalent system that governs the dynamics of $u_{1}, \omega_{1}$ and $\psi_{1}$ :

$$
\begin{aligned}
\partial_{t} u_{1}+u^{r} \partial_{r} u_{1}+u^{z} \partial_{z} u_{1} & =\nu\left(\partial_{r}^{2}+\frac{3}{r} \partial_{r}+\partial_{z}^{2}\right) u_{1}+2 u_{1} \psi_{1 z} \\
\partial_{t} \omega_{1}+u^{r} \partial_{r} \omega_{1}+u^{z} \partial_{z} \omega_{1} & =\nu\left(\partial_{r}^{2}+\frac{3}{r} \partial_{r}+\partial_{z}^{2}\right) \omega_{1}+\left(u_{1}^{2}\right)_{z} \\
-\left(\partial_{r}^{2}+\frac{3}{r} \partial_{r}+\partial_{z}^{2}\right) \psi_{1} & =\omega_{1}
\end{aligned}
$$

where $u^{r}=-r \psi_{1 z}, \quad u^{z}=2 \psi_{1}+r \psi_{1 r}$. Liu and Wang (2006) showed that if $\mathbf{u}$ is a smooth velocity field, then $u^{\theta}, \omega^{\theta}$ and $\psi^{\theta}$ must satisfy the compatibility condition $\left.u^{\theta}\right|_{r=0}=\left.\omega^{\theta}\right|_{r=0}=\left.\psi^{\theta}\right|_{r=0}=0$. Thus $u_{1}, \psi_{1}$ and $\omega_{1}$ are well defined as long as the solution remains smooth.

\subsection{An exact $1 D$ model for 3D Navier-Stokes equations}

Hou and Li (2008a) derived an exact 1D model along the symmetry axis by assuming the solution is more singular along the $z$-direction than along the $r$-direction (i.e., the solution has an locally anisotropic scaling). Along the symmetry axis $r=0$, we have $u^{r}=0, u^{z}=2 \psi_{1}$. Since the solution is more singular along the $z$-direction, one can drop the derivatives along the $r$-direction to the leading order in the reformulated Navier-Stokes equations (note that $\frac{3}{r} \partial_{r}$ is of the same order as $\partial_{r}^{2}$ ). This gives rise to the following 
1D model:

$$
\begin{aligned}
\left(u_{1}\right)_{t}+2 \psi_{1}\left(u_{1}\right)_{z} & =\nu\left(u_{1}\right)_{z z}+2\left(\psi_{1}\right)_{z} u_{1} \\
\left(\omega_{1}\right)_{t}+2 \psi_{1}\left(\omega_{1}\right)_{z} & =\nu\left(\omega_{1}\right)_{z z}+\left(u_{1}^{2}\right)_{z} \\
-\left(\psi_{1}\right)_{z z} & =\omega_{1} .
\end{aligned}
$$

Note that the system $(3.17)-(3.19)$ is already a closed system. Let $\tilde{u}=u_{1}$, $\tilde{v}=-\left(\psi_{1}\right)_{z}$, and $\tilde{\psi}=\psi_{1}$. By integrating (3.18) with respect to $z$, one can further reduce the above system to

$$
\begin{aligned}
& (\tilde{u})_{t}+2 \tilde{\psi}(\tilde{u})_{z}=\nu(\tilde{u})_{z z}-2 \tilde{v} \tilde{u}, \\
& (\tilde{v})_{t}+2 \tilde{\psi}(\tilde{v})_{z}=\nu(\tilde{v})_{z z}+(\tilde{u})^{2}-(\tilde{v})^{2}+c(t),
\end{aligned}
$$

where $\tilde{v}=-(\tilde{\psi})_{z}, \tilde{v}_{z}=\tilde{\omega}$, and $c(t)$ is an integration constant to enforce the mean of $\tilde{v}$ equal to zero. If we assume that the solution is periodic with respect to $z$ with period 1 , the integration constant $c(t)$ is equal to $3 \int_{0}^{1}(\tilde{v})^{2} \mathrm{~d} z-\int_{0}^{1}(\tilde{u})^{2} \mathrm{~d} z$.

A surprising result is that the above $1 \mathrm{D}$ model is exact. This is stated in the following theorem.

Theorem 3.1. Let $u_{1}, \psi_{1}$ and $\omega_{1}$ be the solution of the $1 \mathrm{D}$ model (3.17)(3.19) and define

$$
u^{\theta}(r, z, t)=r u_{1}(z, t), \quad \omega^{\theta}(r, z, t)=r \omega_{1}(z, t), \quad \psi^{\theta}(r, z, t)=r \psi_{1}(z, t) .
$$

Then $\left(u^{\theta}(r, z, t), \omega^{\theta}(r, z, t), \psi^{\theta}(r, z, t)\right)$ is an exact solution of the 3D NavierStokes equations.

Theorem 3.1 tells us that the 1D model (3.17)-(3.19) preserves some essential nonlinear structure of the 3D axisymmetric Navier-Stokes equations.

\subsection{Properties of the model equation}

In this section, we will study some properties of the 1D model. We first consider the properties of some further simplified models obtained from these equations. Both numerical and analytical studies are presented for these simplified models. Based on the understanding of the simplified models, we prove the global existence of the full 1D model.

\section{The ODE model}

To start with, we consider an ODE model by ignoring the convection and diffusion term:

$$
\begin{aligned}
& (\tilde{u})_{t}=-2 \tilde{v} \tilde{u}, \\
& (\tilde{v})_{t}=(\tilde{u})^{2}-(\tilde{v})^{2},
\end{aligned}
$$

with initial condition $\tilde{u}(0)=\tilde{u}_{0}$ and $\tilde{v}(0)=\tilde{v}_{0}$. 
Clearly, if $\tilde{u}_{0}=0$, then $\tilde{u}(t)=0$ for all $t>0$. In this case, the equation for $\tilde{v}$ is decoupled from $\tilde{u}$ completely, and will blow up in finite time if $\tilde{v}_{0}<0$. In fact, if $\tilde{v}_{0}<0$ and $\tilde{u}_{0}$ is very small, then the solution can experience very large growth dynamically. The growth can be made arbitrarily large if we choose $\tilde{u}_{0}$ to be arbitrarily small. However, the special nonlinear structure of the ODE system has an interesting cancellation property which has a stabilizing effect on the solution for large times. This is described by the following theorem.

Theorem 3.2. Assume that $\tilde{u}_{0} \neq 0$. Then the solution $(\tilde{u}(t), \tilde{v}(t))$ of the ODE system (3.22)-(3.23) exists for all times. Moreover, we have

$$
\lim _{t \rightarrow+\infty} \tilde{u}(t)=0, \quad \lim _{t \rightarrow+\infty} \tilde{v}(t)=0 .
$$

Proof. Inspired by the work of Constantin, Lax and Majda (1985), we make the following change of variables: $w=\tilde{u}+i \tilde{v}$. Then the ODE system (3.22)-(3.23) is reduced to the following complex nonlinear ODE:

$$
\frac{\mathrm{d} w}{\mathrm{~d} t}=\mathrm{i} w^{2}, \quad w(0)=w_{0},
$$

which can be solved analytically. The solution has the form

$$
w(t)=\frac{w_{0}}{1-\mathrm{i} w_{0} t} .
$$

In terms of the original variables, we have

$$
\begin{gathered}
\tilde{u}(t)=\frac{\tilde{u}_{0}\left(1+\tilde{v}_{0} t\right)-\tilde{u}_{0} \tilde{v}_{0} t}{\left(1+\tilde{v}_{0} t\right)^{2}+\left(\tilde{u}_{0} t\right)^{2}}, \\
\tilde{v}(t)=\frac{\tilde{v}_{0}\left(1+\tilde{v}_{0} t\right)+\tilde{u}_{0}^{2} t}{\left(1+\tilde{v}_{0} t\right)^{2}+\left(\tilde{u}_{0} t\right)^{2}} .
\end{gathered}
$$

It is clear from (3.27)-(3.28) that the solution of the ODE system (3.22)(3.23) exists for all times and decays to zero as $t \rightarrow+\infty$ as long as $\tilde{u}_{0} \neq 0$. This completes the proof of Theorem 3.2.

As we can see from (3.27)-(3.28), the solution can grow very fast in a very short time if $\tilde{u}_{0}$ is small, but $\tilde{v}_{0}$ is large and negative. For example, if we let $\tilde{v}_{0}=-1 / \epsilon$ and $\tilde{u}_{0}=\epsilon$ for $\epsilon>0$ small, we obtain at $t=\epsilon$

$$
\tilde{u}(\epsilon)=1 / \epsilon^{3}, \quad \tilde{v}(\epsilon)=1 / \epsilon .
$$

We can see that within $\epsilon$ time, $\tilde{u}$ grows from its initial value of order $\epsilon$ to $O\left(\epsilon^{-3}\right)$, a factor of $\epsilon^{-4}$ amplification.

The key ingredient in obtaining the global existence in Theorem 3.2 is that the coefficient on the right-hand side of (3.22) is less than -1 . For this ODE system, there are two distinguished phases. In the first phase, if $\tilde{v}$ is negative and large in magnitude, but $\tilde{u}$ is small, then $\tilde{v}$ can experience 


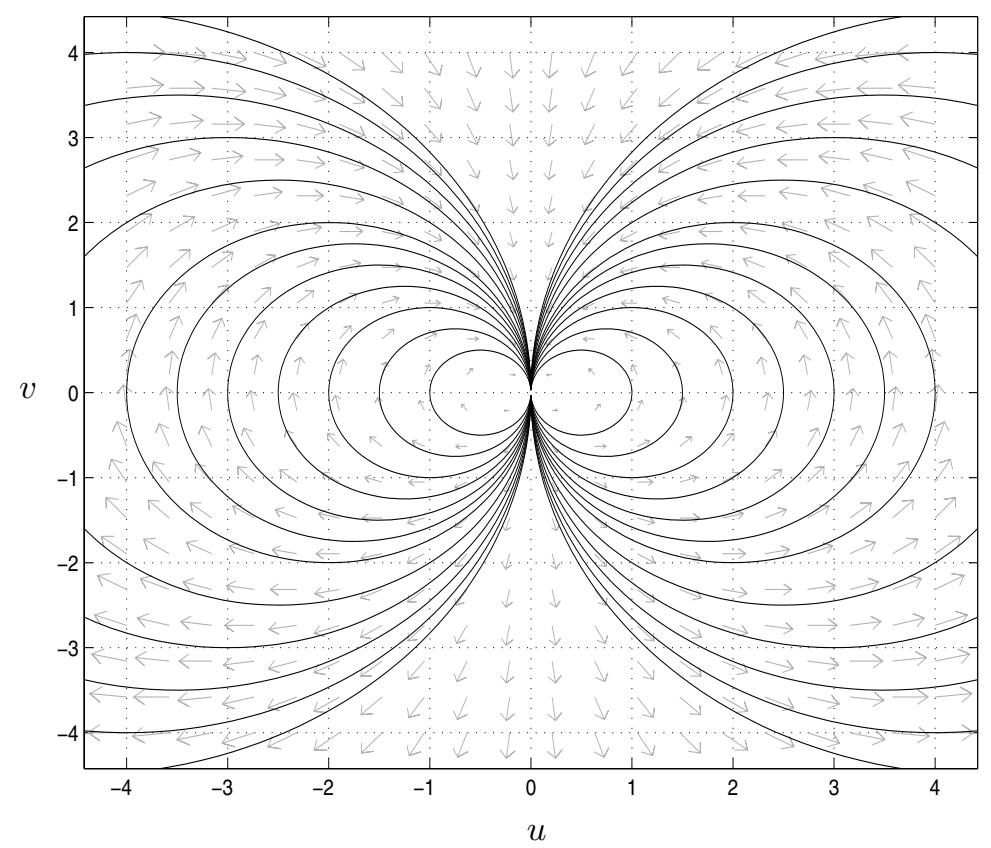

Figure 3.1. The phase diagram for the ODE system.

tremendous dynamic growth, which is essentially governed by

$$
\tilde{v}_{t}=-\tilde{v}^{2} \text {. }
$$

However, as $\tilde{v}$ becomes very large and negative, it will induce a rapid growth in $\tilde{u}$. The nonlinear structure of the ODE system is such that $\tilde{u}$ will eventually grow even faster than $\tilde{v}$ and force $(\tilde{u})^{2}-(\tilde{v})^{2}<0$ in the second phase. From this time on, $\tilde{v}$ will increase in time and eventually become positive. Once $\tilde{v}$ becomes positive, the nonlinear term, $-\tilde{v}^{2}$, becomes stabilizing for $\tilde{v}$. Similarly, the nonlinear term, $-2 \tilde{u} \tilde{v}$, becomes stabilizing for $\tilde{u}$. This subtle dynamic stability property of the ODE system can be best illustrated by the phase diagram in Figure 3.1.

\section{The reaction-diffusion model}

In this subsection, we consider the reaction-diffusion system:

$$
\begin{aligned}
& (\tilde{u})_{t}=\nu \tilde{u}_{z z}-2 \tilde{v} \tilde{u} \\
& (\tilde{v})_{t}=\nu \tilde{v}_{z z}+(\tilde{u})^{2}-(\tilde{v})^{2} .
\end{aligned}
$$

As we can see for the corresponding ODE system, the structure of the nonlinearity plays an essential role in obtaining global existence. Intuitively, one may think that the diffusion term would help to stabilize the dynamic 
growth induced by the nonlinear terms. However, because the nonlinear ODE system in the absence of viscosity is very unstable, the diffusion term can actually have a destabilizing effect. Below we demonstrate this somewhat surprising fact through careful numerical experiments.

In Figures 3.2-3.4, we plot a time sequence of solutions for the above reaction-diffusion system with the following initial data:

$$
\tilde{u}_{0}(z)=\epsilon(2+\sin (2 \pi z)), \quad \tilde{v}_{0}(z)=-\frac{1}{\epsilon}-\sin (2 \pi z),
$$

where $\epsilon=0.001$. For this initial condition, the solution is periodic in $z$ with period one. We use a pseudo-spectral method to discretize the coupled system (3.29)-(3.30) in space and use the simple forward Euler discretization for the nonlinear terms and the backward Euler discretization for the diffusion term. In order to resolve the nearly singular solution structure, we use $N=32,768$ grid points with an adaptive time step satisfying

$$
\Delta t_{n}\left(\left|\max \left\{\tilde{u}^{n}\right\}\right|+\left|\min \left\{\tilde{u}^{n}\right\}\right|+\left|\max \left\{\tilde{v}^{n}\right\}\right|+\left|\min \left\{\tilde{v}^{n}\right\}\right|\right) \leq 0.01,
$$

where $\tilde{u}^{n}$ and $\tilde{v}^{n}$ are the numerical solution at time $t_{n}$ and $t_{n}=t_{n-1}+\Delta t_{n-1}$ with the initial time stepsize $\Delta t_{0}=0.01 \epsilon$. During the time iterations, the smallest time step is as small as $O\left(10^{-10}\right)$.

From Figure 3.2, we can see that the magnitude of the solution $\tilde{v}$ increases rapidly by a factor of 150 within a very short time $(t=0.00099817)$. As the solution $\tilde{v}$ becomes large and negative, the solution $\tilde{u}$ increases much more rapidly than $\tilde{v}$. By time $t=0.0010042, \tilde{u}$ has increased to about $2.5 \times 10^{8}$ from its initial condition, which is of magnitude $10^{-3}$. This is a factor of $2.5 \times 10^{11}$ increase. At this time, the minimum of $\tilde{v}$ has reached $-2 \times 10^{8}$. Note that since $\tilde{u}$ has outgrown $\tilde{v}$ in magnitude, the nonlinear term, $\tilde{u}^{2}-\tilde{v}^{2}$, on the right-hand side of the $\tilde{v}$-equation has changed sign. This causes the solution $\tilde{v}$ to split. By the time $t=0.001004314$ (see Figure 3.3), both $\tilde{u}$ and $\tilde{v}$ have split and settled down to two relatively stable travelling wave solutions. The wave on the left will travel to the left while the wave on the right will travel to the right. Due to the periodicity in $z$, the two travelling waves approach each other from the right side of the domain. The 'collision' of these two travelling waves tends to annihilate each other. In particular, the negative part of $\tilde{v}$ is effectively eliminated during this nonlinear interaction. By the time $t=0.00100603$ (see Figure 3.4), the solution $\tilde{v}$ becomes all positive. Once $\tilde{v}$ becomes positive, the effect of nonlinearity becomes stabilizing for both $\tilde{u}$ and $\tilde{v}$, as in the case of the ODE system. From then on, the solution decays rapidly. By $t=0.2007$, the magnitude of $\tilde{u}$ is as small as $5.2 \times 10^{-8}$, and $\tilde{v}$ becomes almost a constant function with value close to 5 . From this time on, $\tilde{u}$ is essentially decoupled from $\tilde{v}$ and will decay like $O(1 / t)$. 
(a)

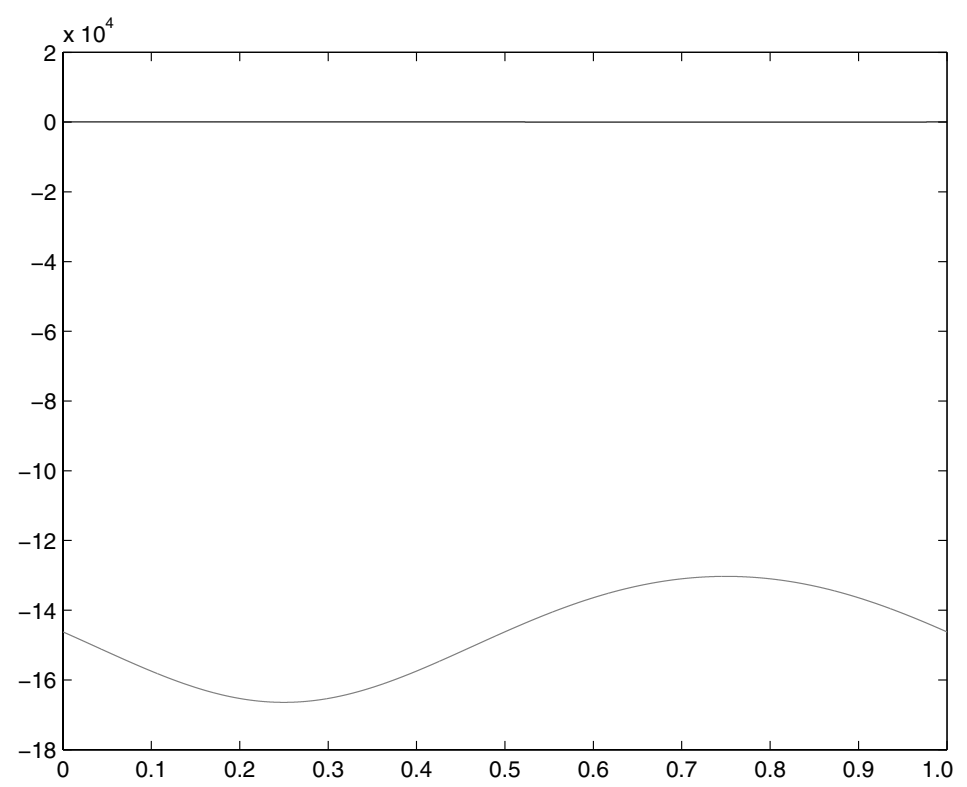

(b)

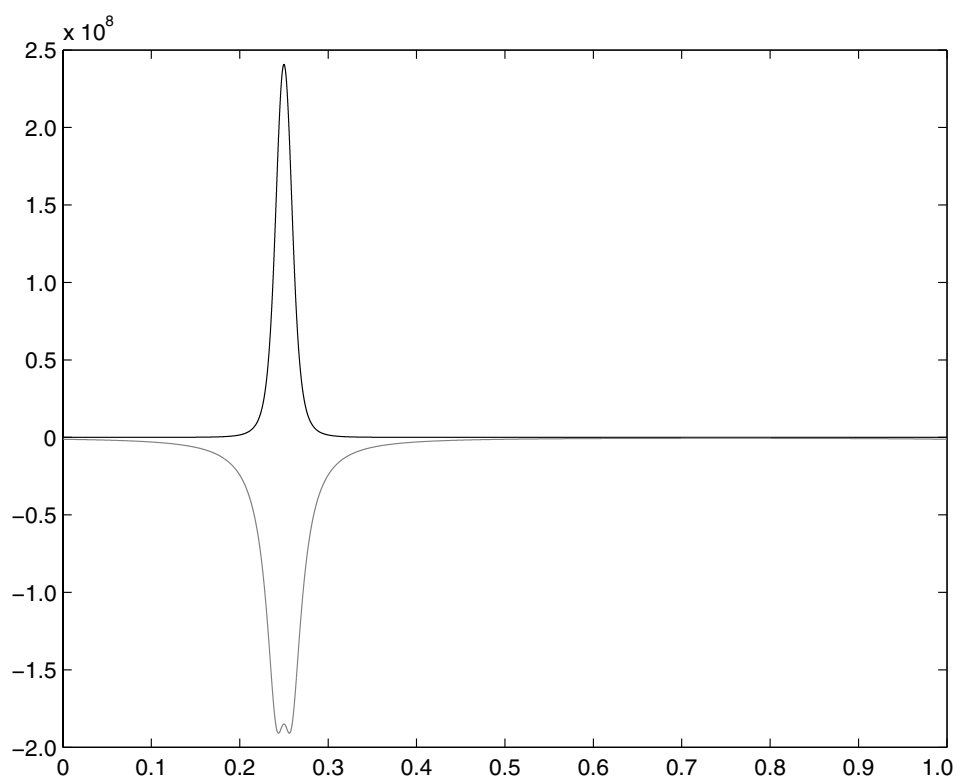

Figure 3.2. The solutions $u$ (dark curve) and $v$ (light curve) at (a) $t=0.00099817$, and (b) $t=0.0010042$, respectively; $N=32768, \nu=1$. 
(a)

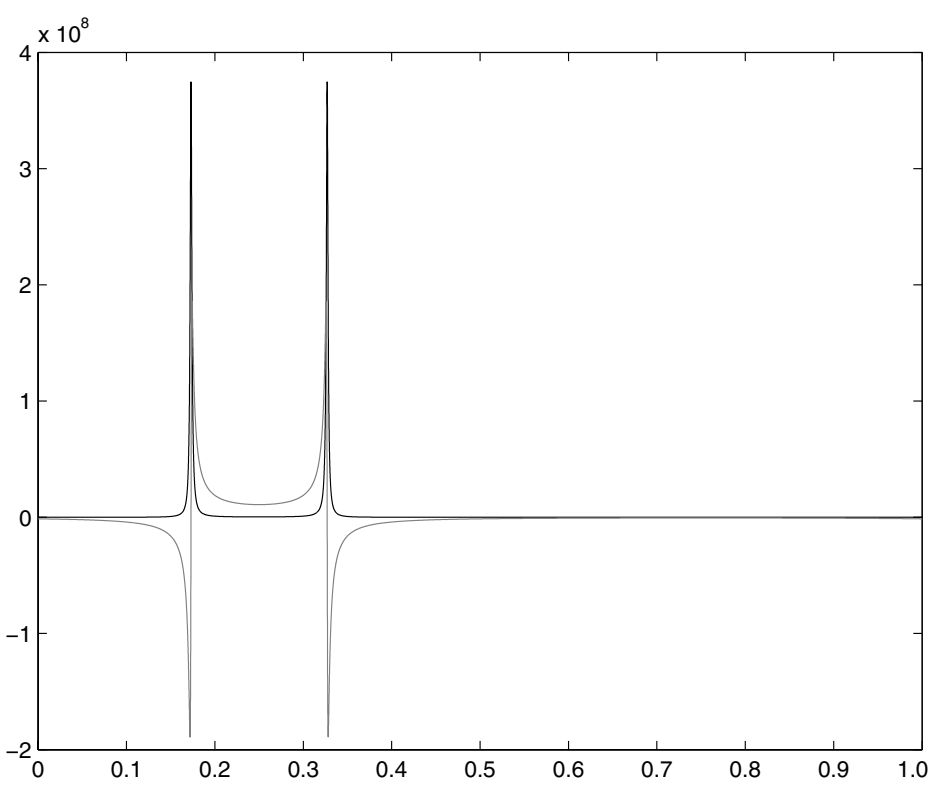

(b)

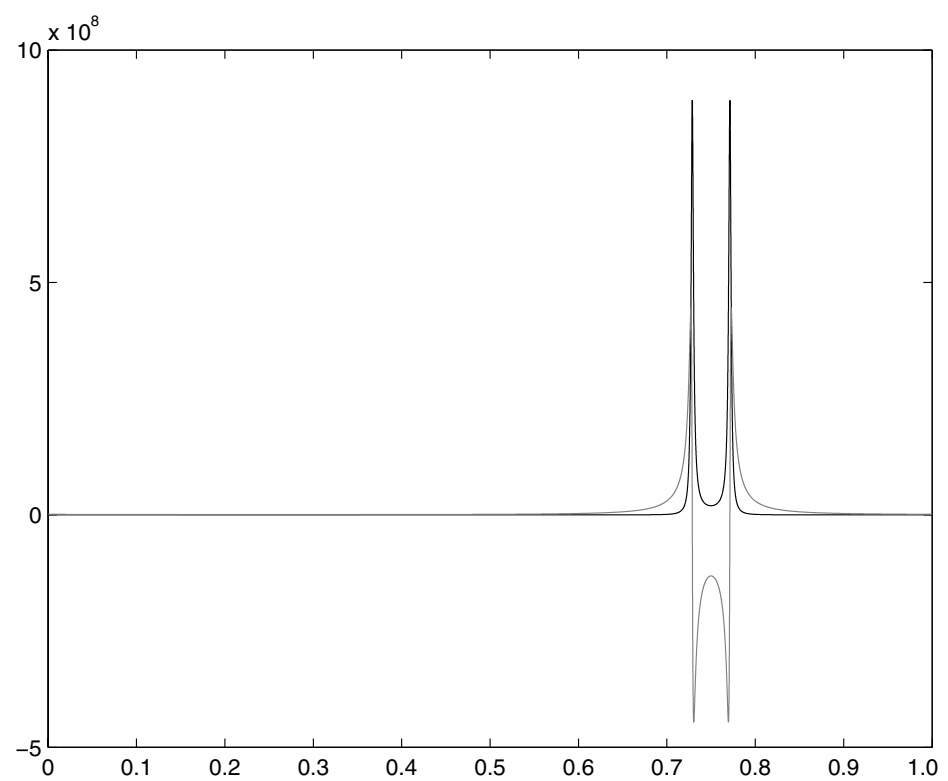

Figure 3.3. The solutions $u$ (dark) and $v$ (light) at (a) $t=0.001004314$, and (b) $t=0.001005862$, respectively; $N=32768, \nu=1$. 
(a)

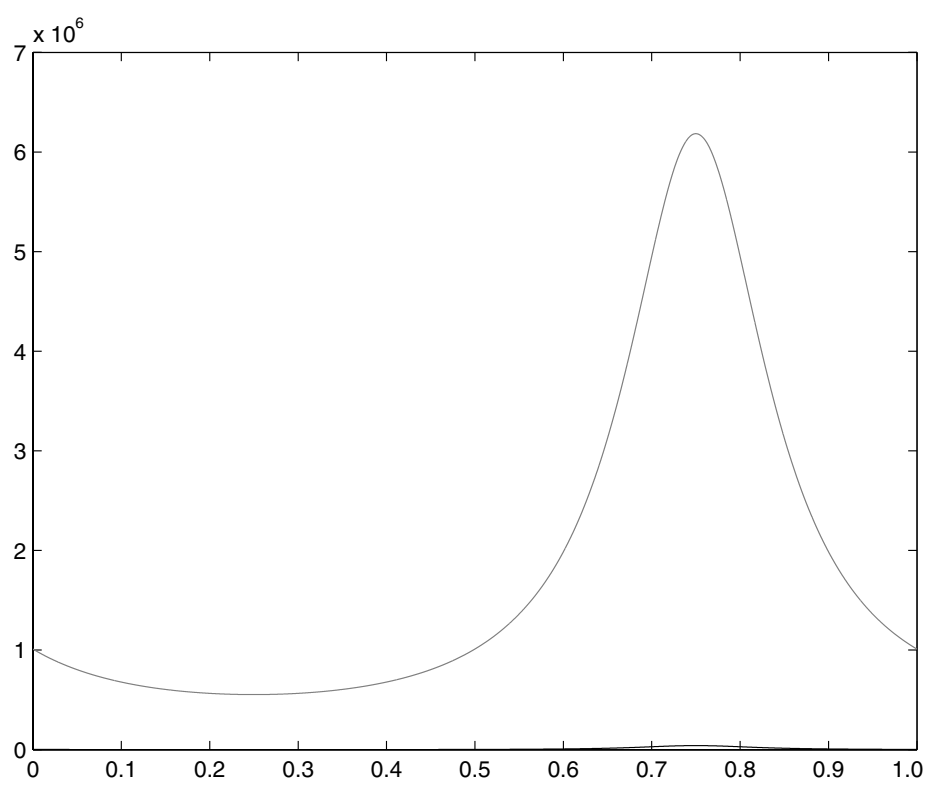

(b)

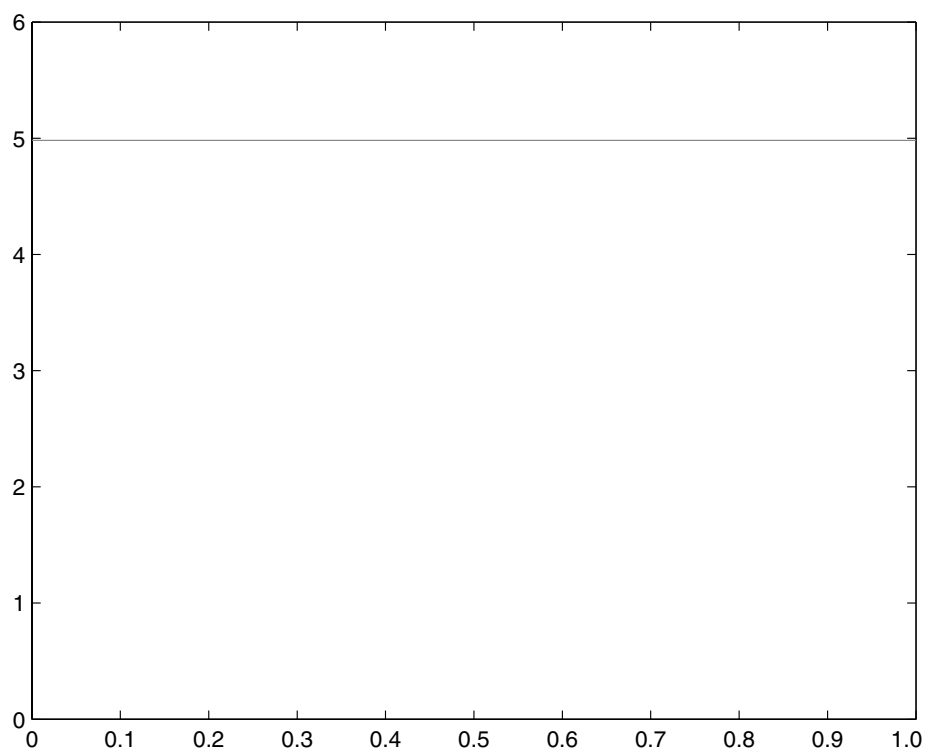

Figure 3.4. The solutions $u$ (dark) and $v$ (light) at (a) $t=0.00100603$, and (b) $t=0.2007$, respectively; $N=32768, \nu=1$. Note that at $t=0.00100603$, the value of $u$ becomes quite small and is very close to the $x$-axis. By $t=0.2007$, the value of $u$ is of the order $5.2 \times 10^{-8}$ and is almost invisible. 
(a)

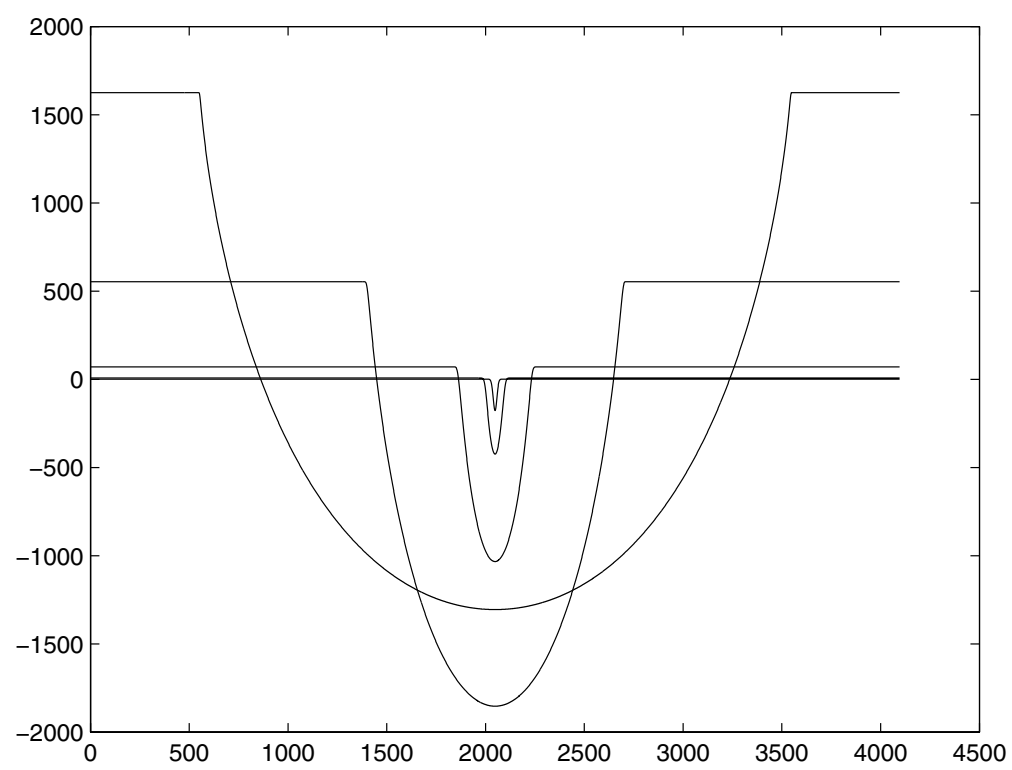

(b)

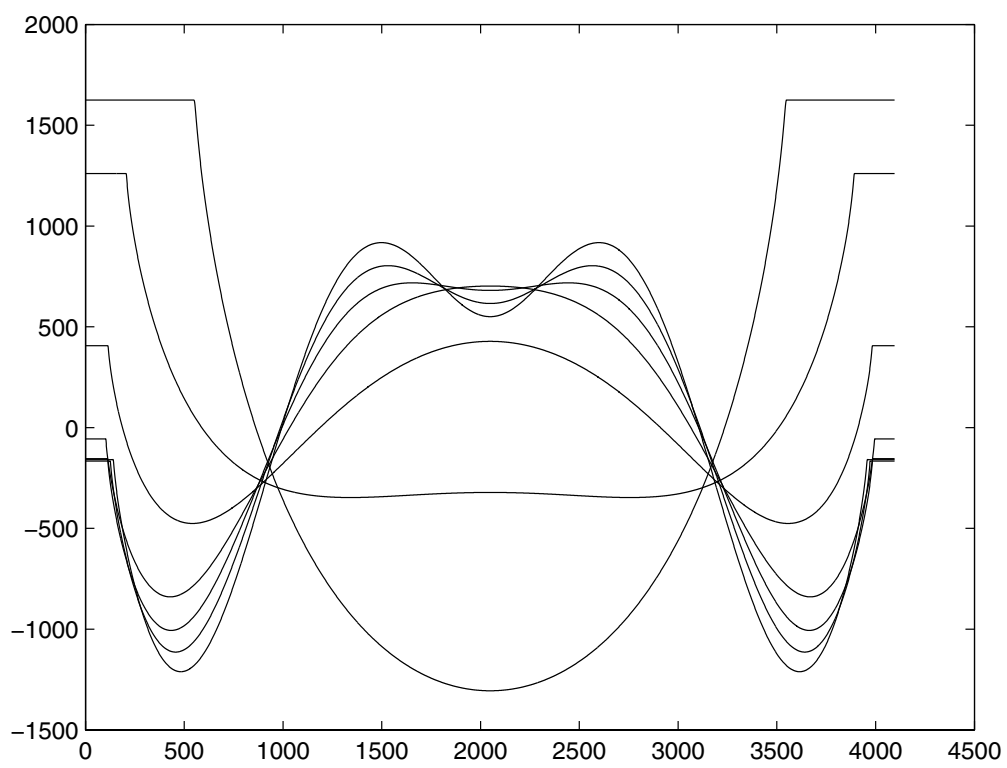

Figure 3.5. The time sequence of $v$ in the Eulerian coordinate, $N=4096, \nu=0$. (a) $t=0,0.0033,0.0048,0.0055,0.0059$, (b) $t=0.0059,0.0062,0.0066,0.007$, $0.0074,0.0078,0.0081$. The solutions are plotted against the number of grid points corresponding to the range $[0,1]$ in physical space. 


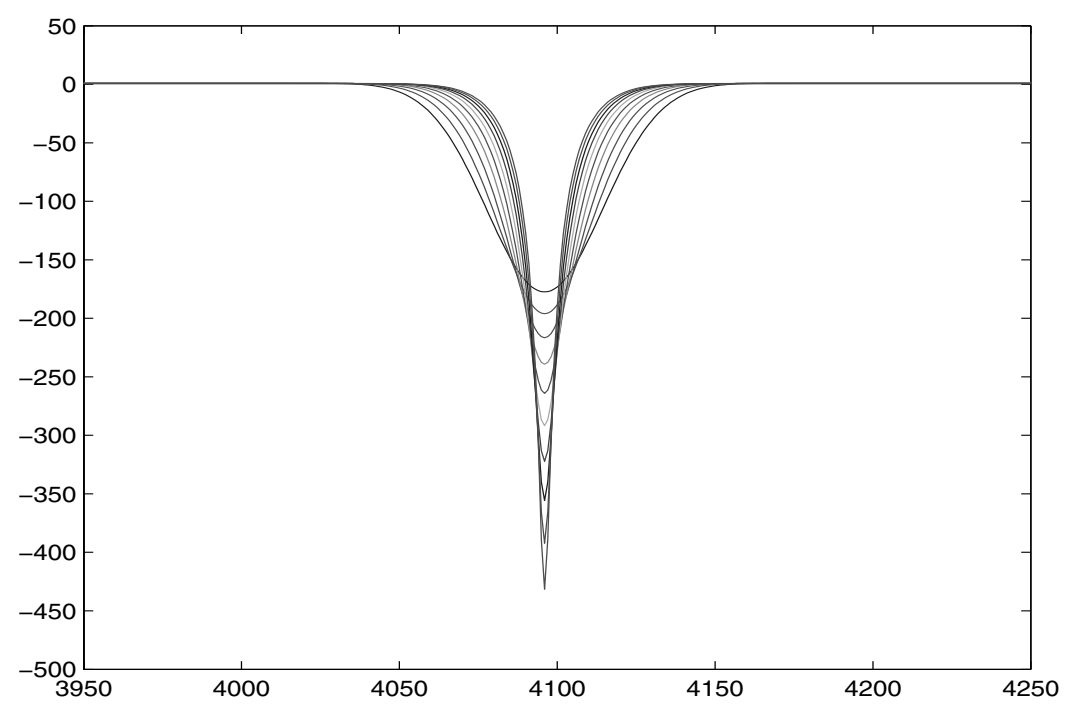

Figure 3.6. The time sequence of solution $v$ in the Lagrangian coordinate by solving the model equation with the wrong sign, $N=8192, \nu=0$. The time sequence is from $t=0$ to 0.0033 corresponding to a sequence of curves from the top to the bottom. The solutions are plotted against the number of grid points corresponding to the range $[0.482,0.519]$ in physical space.

\subsection{Global well-posedness of the full 1D model}

We have also performed numerical studies of the full $1 \mathrm{D}$ model. We find that the solution behaviour of the full 1D model is completely different from the reaction-diffusion model. In particular, the convection term plays an essential role in regularizing the nearly singular behaviour of the reactiondiffusion model. In our numerical computations, we use a pseudo-spectral method to discretize in space and a second-order Runge-Kutta discretization in time with an adaptive time-stepping. The initial data are given by

$$
u(\alpha, 0)=1, \quad v(\alpha, 0)=1-\frac{1}{\delta} \exp ^{-(x-0.5)^{2} / \epsilon},
$$

with $\epsilon=0.00001$ and $\delta=\sqrt{\epsilon \pi}$. In Figure 3.5, we plot a sequence of snapshots of the solution. We see that the solution experiences a similar splitting process as in the reaction-diffusion model. On the other hand, we observe that as the solution $\tilde{v}$ grows large and negative, the initial sharp profile of $\tilde{v}$ becomes defocused and smoother. This is a consequence of the incompressibility of the fluid flow. If we change the sign of the convection velocity from $2 \tilde{\psi}$ to $-2 \tilde{\psi}$, the profile of $\tilde{v}$ becomes focused dynamically and seems to evolve into a focusing finite-time blow-up: see Figure 3.6. 
Based on our numerical studies, we become convinced that the solution of the full 1D model should be regular for all times. However, it is extremely difficult, if not impossible, to prove the global regularity of the 1D model by using an energy type of estimates. If we multiply the $\tilde{u}$-equation by $\tilde{u}$, and the $\tilde{v}$-equation by $\tilde{v}$, and integrate over $z$, we arrive at

$$
\begin{aligned}
& \frac{1}{2} \frac{\mathrm{d}}{\mathrm{d} t} \int_{0}^{1} \tilde{u}^{2} \mathrm{~d} z=-3 \int_{0}^{1}(\tilde{u})^{2} \tilde{v} \mathrm{~d} z-\nu \int_{0}^{1} \tilde{u}_{z}^{2} \mathrm{~d} z \\
& \frac{1}{2} \frac{\mathrm{d}}{\mathrm{d} t} \int_{0}^{1} \tilde{v}^{2} \mathrm{~d} z=\int_{0}^{1} \tilde{u}^{2} \tilde{v} \mathrm{~d} z-3 \int_{0}^{1}(\tilde{v})^{3} \mathrm{~d} z-\nu \int_{0}^{1} \tilde{v}_{z}^{2} \mathrm{~d} z
\end{aligned}
$$

Even for this 1D model, the energy estimate shares the some essential difficulty as the 3D Navier-Stokes equations. It is not clear how to control the nonlinear vortex-stretching-like terms by the diffusion term. On the other hand, if we assume that

$$
\int_{0}^{T}\|\tilde{v}\|_{L^{\infty}} \mathrm{d} t<\infty,
$$

similar to the Beale-Kato-Majda non-blow-up condition for vorticity (Beale, Kato and Majda 1984), then one can easily show that there is no blow-up before $t=T$.

In order to obtain the global regularity of the $1 \mathrm{D}$ model, we need to use a local estimate. The key is to obtain a pointwise estimate for a positive Lyapunov function. Convection is found to play an essential role in cancelling the destabilizing vortex stretching terms. Using this pointwise estimate, we can prove that if the initial conditions for $\tilde{u}$ and $\tilde{v}$ are in $C^{m}$ with $m \geq 1$, then the solution remains in $C^{m}$ for all times.

Theorem 3.3. (Hou and Li 2008a) Assume that $\tilde{u}(z, 0)$ and $\tilde{v}(z, 0)$ are in $C^{m}[0,1]$ with $m \geq 1$ and periodic with period 1 . Then the solution $(\tilde{u}, \tilde{v})$ of the 1D model will be in $C^{m}[0,1]$ for all times and for $\nu \geq 0$.

Proof. The key is to obtain a pointwise estimate a priori for the positive Lyapunov function $\tilde{u}_{z}^{2}+\tilde{v}_{z}^{2}$. Differentiating (3.20)-(3.21) with respect to $z$, we get

$$
\begin{aligned}
&\left(\tilde{u}_{z}\right)_{t}+2 \tilde{\psi}\left(\tilde{u}_{z}\right)_{z}-2 \tilde{v} \tilde{u}_{z}=-2 \tilde{v} \tilde{u}_{z}-2 \tilde{u} \tilde{v}_{z}+\nu\left(\tilde{u}_{z}\right)_{z z}, \\
&\left(\tilde{v}_{z}\right)_{t}+2 \tilde{\psi}\left(\tilde{v}_{z}\right)_{z}-2 \tilde{v} \tilde{v}_{z}=2 \tilde{u} \tilde{u}_{z}-2 \tilde{v} \tilde{v}_{z}+\nu\left(\tilde{v}_{z}\right)_{z z} .
\end{aligned}
$$

Note that the convection term contributes to stability by cancelling one of the nonlinear terms on the right-hand side. This gives

$$
\begin{aligned}
&\left(\tilde{u}_{z}\right)_{t}+2 \tilde{\psi}\left(\tilde{u}_{z}\right)_{z}=-2 \tilde{u} \tilde{v}_{z}+\nu\left(\tilde{u}_{z}\right)_{z z} \\
&\left(\tilde{v}_{z}\right)_{t}+2 \tilde{\psi}\left(\tilde{v}_{z}\right)_{z}=2 \tilde{u} \tilde{u}_{z}+\nu\left(\tilde{v}_{z}\right)_{z z} .
\end{aligned}
$$


Multiplying (3.35) by $2 \tilde{u}_{z}$ and (3.36) by $2 \tilde{v}_{z}$, we obtain

$$
\begin{aligned}
& \left(\tilde{u}_{z}^{2}\right)_{t}+2 \tilde{\psi}\left(\tilde{u}_{z}^{2}\right)_{z}=-4 \tilde{u} \tilde{u}_{z} \tilde{v}_{z}+2 \nu \tilde{u}_{z}\left(\tilde{u}_{z}\right)_{z z}, \\
& \left(\tilde{v}_{z}^{2}\right)_{t}+2 \tilde{\psi}\left(\tilde{v}_{z}^{2}\right)_{z}=4 \tilde{u} \tilde{u}_{z} \tilde{v}_{z}+2 \nu \tilde{v}_{z}\left(\tilde{v}_{z}\right)_{z z} .
\end{aligned}
$$

Now, we add (3.37) to (3.38). Surprisingly, the remaining nonlinear vortex stretching terms cancel each other exactly. We get

$$
\left(\tilde{u}_{z}^{2}+\tilde{v}_{z}^{2}\right)_{t}+2 \tilde{\psi}\left(\tilde{u}_{z}^{2}+\tilde{v}_{z}^{2}\right)_{z}=2 \nu\left(\tilde{u}_{z}\left(\tilde{u}_{z}\right)_{z z}+\tilde{v}_{z}\left(\tilde{v}_{z}\right)_{z z}\right)
$$

Further, we can rewrite equation (3.39) as follows:

$$
\left(\tilde{u}_{z}^{2}+\tilde{v}_{z}^{2}\right)_{t}+2 \tilde{\psi}\left(\tilde{u}_{z}^{2}+\tilde{v}_{z}^{2}\right)_{z}=\nu\left(\tilde{u}_{z}^{2}+\tilde{v}_{z}^{2}\right)_{z z}-2 \nu\left[\left(\tilde{u}_{z z}\right)^{2}+\left(\tilde{v}_{z z}\right)^{2}\right] .
$$

Now it is easy to see that $\left(\tilde{u}_{z}^{2}+\tilde{v}_{z}^{2}\right)$ satisfies a maximum principle for all $\nu \geq 0$ :

$$
\left\|\tilde{u}_{z}^{2}+\tilde{v}_{z}^{2}\right\|_{L^{\infty}} \leq\left\|\left(\tilde{u}_{0}\right)_{z}^{2}+\left(\tilde{v}_{0}\right)_{z}^{2}\right\|_{L^{\infty}} .
$$

It is worth emphasizing that the cancellation between the convection term and the vortex stretching term takes place at the inviscid level. Viscosity does not play an essential role here. Since $\tilde{v}$ has zero mean, the Poincaré inequality implies that $\|\tilde{v}\|_{L^{\infty}} \leq C_{0}$, with $C_{0}$ defined by

$$
C_{0}=\left\|\left(\left(\tilde{u}_{0}\right)_{z}^{2}+\left(\tilde{v}_{0}\right)_{z}^{2}\right)^{\frac{1}{2}}\right\|_{L^{\infty}} .
$$

The boundedness of $\tilde{u}$ follows from the bound on $\tilde{v}$, that is, $\|\tilde{u}(t)\|_{L^{\infty}} \leq$ $\left\|\tilde{u}_{0}\right\|_{L^{\infty}} \exp \left(2 C_{0} t\right)$. The higher-order regularity follows from the standard estimates. This proves Theorem 3.3.

\subsection{Construction of a family of $3 D$ globally smooth solutions}

We can use the solution from the 1D model to construct a family of globally smooth solutions for the 3D axisymmetric Navier-Stokes equations with large initial data of finite energy. We remark that a special feature of this family of globally smooth solutions is that the solution can potentially develop very large dynamic growth and it violates the smallness condition required by classical global existence results (Constantin and Foias 1988, Temam 2001).

Theorem 3.4. (Hou and Li 2008a) Let $\phi(r)$ be a smooth cut-off function and $u_{1}, \omega_{1}$ and $\psi_{1}$ be the solution of the $1 \mathrm{D}$ model. Define

$$
\begin{aligned}
u^{\theta}(r, z, t) & =r u_{1}(z, t) \phi(r)+\tilde{u}(r, z, t), \\
\omega^{\theta}(r, z, t) & =r \omega_{1}(z, t) \phi(r)+\tilde{\omega}(r, z, t), \\
\psi^{\theta}(r, z, t) & =r \psi_{1}(z, t) \phi(r)+\tilde{\psi}(r, z, t) .
\end{aligned}
$$


Then there exists a family of globally smooth functions $\tilde{u}, \tilde{\omega}$ and $\tilde{\psi}$ such that $u^{\theta}, \omega^{\theta}$ and $\psi^{\theta}$ are globally smooth solutions of the 3D Navier-Stokes equations with finite energy.

\section{Stabilizing effect of convection for 3D Navier-Stokes}

Hou and Lei $(2009 b)$ studied the stabilizing effect of the convection term in the 3D incompressible Euler or Navier-Stokes equations using a new 3D model. This model was derived from the reformulated Navier-Stokes equations. It shares many properties with the 3D Euler or Navier-Stokes equations. First of all, it has the same nonlinear vortex stretching term. Secondly, it has the same type of a priori energy identity. Thirdly, almost all the existing non-blow-up criteria for the 3D Euler or Navier-Stokes equations are also valid for our model. A 3D model that satisfies all these properties seems hard to find in general. But in terms of the equations for the new variables, $u_{1}, \omega_{1}$, and $\psi_{1}$, we obtain our $3 \mathrm{D}$ model equations by simply dropping the convective term from the reformulated Navier-Stokes equations (3.16):

$$
\begin{aligned}
\partial_{t} u_{1} & =\nu\left(\partial_{r}^{2}+\frac{3}{r} \partial_{r}+\partial_{z}^{2}\right) u_{1}+2 u_{1} \psi_{1 z}, \\
\partial_{t} \omega_{1} & =\nu\left(\partial_{r}^{2}+\frac{3}{r} \partial_{r}+\partial_{z}^{2}\right) \omega_{1}+\left(u_{1}^{2}\right)_{z}, \\
& -\left(\partial_{r}^{2}+\frac{3}{r} \partial_{r}+\partial_{z}^{2}\right) \psi_{1}=\omega_{1} .
\end{aligned}
$$

Note that (4.1) is already a closed system. The main difference between our $3 \mathrm{D}$ model and the Navier-Stokes equations is that we neglect the convection term in our model. If we add the convection term back to our 3D model, we will recover the Navier-Stokes equations.

Below we will summarize some important properties of the model equations (4.1).

\subsection{Properties of the $3 D$ model}

This 3D model shares many important properties with the axisymmetric Navier-Stokes equations. First of all, one can define an incompressible velocity field for the $3 \mathrm{D}$ model,

$$
\begin{gathered}
\mathbf{u}(t, \mathbf{x})=u^{r}(t, r, z) e_{r}+u^{\theta}(t, r, z) e_{\theta}+u^{z}(t, r, z) e_{z}, \\
u^{\theta}=r u_{1}, u^{r}=-r \psi_{1 z}, \quad u^{z}=2 \psi_{1}+r \psi_{1 r},
\end{gathered}
$$

where $\mathbf{x}=(x, y, z), r=\sqrt{x^{2}+y^{2}}$. It is easy to check that

$$
\nabla \cdot \mathbf{u}=\partial_{r} u^{r}+\partial_{z} u^{z}+\frac{u^{r}}{r}=0,
$$


which is the same as the original Navier-Stokes equations.

Furthermore, Hou and Lei (2009b) proved the following energy identity for the 3D model.

Theorem 4.1. (Energy identity (Hou and Lei 2009b)) The strong solution of (4.1) satisfies

$$
\frac{1}{2} \frac{\mathrm{d}}{\mathrm{d} t} \int\left(\left|u_{1}\right|^{2}+2\left|D \psi_{1}\right|^{2}\right) r^{3} \mathrm{~d} r \mathrm{~d} z+\nu \int\left(\left|D u_{1}\right|^{2}+2\left|D^{2} \psi_{1}\right|^{2}\right) r^{3} \mathrm{~d} r \mathrm{~d} z=0,
$$

Here $D$ is the first-order derivative operator defined in $\mathbb{R}^{5}$.

This energy identity is equivalent to that of the Navier-Stokes equations, which has the form

$$
\frac{1}{2} \frac{\mathrm{d}}{\mathrm{d} t} \int\left(\left|u_{1}\right|^{2}+\left|D \psi_{1}\right|^{2}\right) r^{3} \mathrm{~d} r \mathrm{~d} z+\nu \int\left(\left|D u_{1}\right|^{2}+\left|D^{2} \psi_{1}\right|^{2}\right) r^{3} \mathrm{~d} r \mathrm{~d} z=0 .
$$

Another result obtained by Hou and Lei is a non-blow-up criterion of the 3D model equations (4.1), which is an analogue of the Beale-Kato-Majda (BKM) result for the 3D Euler and Navier-Stokes equations. For the 3D Euler and Navier-Stokes equations, the BKM non-blow-up criterion states that the solution $\mathbf{u}$ blows up at time $T<\infty$ if and only if the accumulation of vorticity $\int_{0}^{T}\left\|\nabla_{x} \times \mathbf{u}\right\|_{L^{\infty}\left(\mathbb{R}^{3}\right)} \mathrm{d} t$ is infinite (Beale, Kato and Majda 1984). The BKM non-blow-up criterion was later improved by Kozono and Taniuchi (2000), who proved that the $\|\cdot\|_{L^{\infty}}$-norm can be replaced by the norm in the BMO space. This generalization is interesting because some crucial Sobolev embedding theorems can be applied to the BMO space, but not to the $L^{\infty}$-space. A non-blow-up result formulated in terms of the BMO space has a broader range of applications.

Theorem 4.2. (A non-blow-up criterion of Beale-Kato-Majda type (Hou and Lei 2009b)) A smooth solution $\left(u_{1}, \omega_{1}, \psi_{1}\right)$ of the model (4.1) for $0 \leq t<T$ blows up at time $t=T$ if and only if

$$
\int_{0}^{T}\|\nabla \times \mathbf{u}\|_{\mathrm{BMO}\left(\mathbb{R}^{3}\right)} \mathrm{d} t=\infty
$$

where $\mathbf{u}$ is defined in (4.2)-(4.3).

There have been many results on the global regularity of the solutions of the 3D Navier-Stokes equations under some additional conditions imposed on the solution. In particular, the papers of Prodi (1959) and Serrin (1963) gave the following non-blow-up criterion for the solution of the 3D Navier-Stokes equations: Any Leray-Hopf solution $u$ to the $3 D$ NavierStokes equations on $[0, T]$ is smooth on $[0, T]$ if $\|\mathbf{u}\|_{L_{t}^{q} L_{x}^{p}\left([0, T] \times \mathbb{R}^{3}\right)}<\infty$ for 
some $p, q$ satisfying $(3 / p)+(2 / q) \leq 1,3<p \leq \infty$. A local version was later established by Serrin $(1962)$ for $(3 / p)+(2 / q)<1$ and by Struwe (1988) for $(3 / p)+(2 / q)=1$. The highly non-trivial end-point case of $p=3$ was recently established by Iskauriaza, Seregin and Sverak (2003).

To demonstrate the similarity between the 3D model equations (4.1) and the axisymmetric Navier-Stokes equations, Hou and Lei proved a non-blowup criterion of the Prodi-Serrin type for their model.

Theorem 4.3. (A non-blow-up criterion of Prodi-Serrin type (Hou and Lei 2009b)) A weak solution $\left(u_{1}, \omega_{1}, \psi_{1}\right)$ of the model (4.1) is smooth on $[0, T] \times \mathbb{R}^{3}$ provided that

$$
\left\|u^{\theta}\right\|_{L_{t}^{q} L_{x}^{p}\left([0, T] \times \mathbb{R}^{3}\right)}<\infty
$$

for some $p, q$ satisfying $\frac{3}{p}+\frac{2}{q} \leq 1$ with $3<p \leq \infty$ and $2 \leq q<\infty$.

Finally, Hou and Lei (2009a) studied the local behaviour of the solutions to the 3D model equations and established an analogue of the CaffarelliKohn-Nirenberg partial regularity theory (Caffarelli et al. 1982) for their model. They proved that for any suitable weak solution of the 3D model in an open set in space-time, the one-dimensional Hausdorff measure of the associated singular set is zero. The proof of this partial regularity result is similar in spirit to that of Lin (1998), but there are some new technical difficulties associated with the 3D model. One of the difficulties is in handling the singularity induced by the cylindrical coordinates. This makes it difficult to analyse the partial regularity of the $3 \mathrm{D}$ model in $\mathbb{R} \times \mathbb{R}^{3}$. To overcome this difficulty, they performed their partial regularity analysis in $\mathbb{R} \times \mathbb{R}^{5}$. By working in $\mathbb{R}^{5}$, they avoided the difficulty associated with the coordinate singularity.

Another difficulty in obtaining our partial regularity result is that we do not have an evolution equation for the entire velocity field. We need to reformulate the model in terms of a new vector variable. This new variable can be considered as a 'generalized velocity field' in $\mathbb{R}^{5}$. We remark that the partial regularity theory for Navier-Stokes equations in $\mathbb{R}^{5}$ is still open due to the lack of certain compactness. When formulating the 3D model in $\mathbb{R} \times \mathbb{R}^{5}$, they found a 3D structure which has the same scaling as that of the 3D Navier-Stokes equations. This is why the partial regularity analysis can be carried out for the 3D model in $\mathbb{R} \times \mathbb{R}^{5}$ using a strategy similar to that of Lin (1998).

Theorem 4.4. (An analogue of Caffarelli-Kohn-Nirenberg partial regularity result (Hou and Lei 2009a) For any suitable weak solution of the 3D model equations (4.1) on an open set in space-time, the onedimensional Hausdorff measure of the associated singular set is zero. 


\subsection{Potential singularity formation of the $3 D$ model}

Despite the striking similarity at the theoretical level between the 3D model and the Navier-Stokes equations, the former displays a completely different behaviour from the full Navier-Stokes equations. In the next subsection, we will present numerical evidence which seems to support that the model may develop a potential finite-time singularity from smooth initial data with finite energy. Before we do that, we would like to gain some understanding at the theoretical level why the 3D model may develop a finite-time singularity. For this purpose, we consider the inviscid model by setting $\nu=0$ in (4.1):

$$
\begin{gathered}
\partial_{t} u_{1}=2 u_{1} \psi_{1 z}, \\
\partial_{t} \omega_{1}=\left(u_{1}^{2}\right)_{z}, \\
-\left(\partial_{r}^{2}+\frac{3}{r} \partial_{r}+\partial_{z}^{2}\right) \psi_{1}=\omega_{1} .
\end{gathered}
$$

If we let $v=\log \left(u_{1}^{2}\right)$, then we can further reduce the $3 \mathrm{D}$ model to the following non-local nonlinear wave equation:

$$
v_{t t}=4\left(\left(-\Delta_{5}\right)^{-1} \mathrm{e}^{v}\right)_{z z},
$$

where $-\Delta_{5}=-\left(\partial_{r}^{2}+\frac{3}{r} \partial_{r}+\partial_{z}^{2}\right)$, and $\int \mathrm{e}^{v} r^{3} \mathrm{~d} r \mathrm{~d} z \leq C_{0}$. Note that $\left(-\Delta_{5}\right)^{-1}$ is a positive operator. If we were to omit $\left(-\Delta_{5}\right)^{-1}$ from (4.10), we would obtain a $1 \mathrm{D}$ nonlinear wave equation, $v_{t t}=4\left(\mathrm{e}^{v}\right)_{z z}$, or

$$
v_{t t}=4 \mathrm{e}^{v} v_{z z}+4 \mathrm{e}^{v}\left(v_{z}\right)^{2},
$$

which we expect to develop a finite-time singularity.

\subsection{Special blow-up solutions of the $3 D$ model}

We can construct a special class of blow-up solutions by letting $u_{1}=z \tilde{u}(r, t)$, $\omega_{1}=z \tilde{\omega}(r, t)$, and $\psi_{1}=z \tilde{\psi}(r, t)$. Then it is easy to derive the following system for $\tilde{u}(r, t), \tilde{\omega}(r, t)$, and $\tilde{\psi}(r, t)$ :

$$
\begin{gathered}
\partial_{t} \tilde{u}=2 \tilde{\psi} \tilde{u}+\nu\left(\partial_{r}^{2}+\frac{3}{r} \partial_{r}\right) \tilde{u}, \\
\partial_{t} \tilde{\omega}=2 \tilde{u}^{2}+\nu\left(\partial_{r}^{2}+\frac{3}{r} \partial_{r}\right) \tilde{\omega}, \\
-\left(\partial_{r}^{2}+\frac{3}{r} \partial_{r}\right) \tilde{\psi}=\tilde{\omega} .
\end{gathered}
$$

Note that the nonlinear terms become local and quadratic. It is easy to show that if the initial data are positive, then the solution of (4.12) will remain positive for all times. Using this property, we can prove that the above system has finite-time blow-up solutions. However, such singular solutions have infinite energy unless we introduce a cut-off along the $z$-direction. 
We remark that Constantin (1986) has constructed a family of finite-time blow-up solutions to the distorted Euler equations:

$$
(\nabla \mathbf{u})_{t}+(\nabla \mathbf{u})^{2}+\nabla \nabla p=0,
$$

where $-\Delta p=\operatorname{Tr}\left((\nabla \mathbf{u})^{2}\right)$. We note that $\mathbf{u}$ is no longer divergence-free (in fact, there is no evolution equation for $\mathbf{u}$ ), and the model does not conserve energy. Moreover, the blow-up solution has infinite energy.

\subsection{Numerical evidence for a potential finite-time singularity}

In this subsection, we present numerical evidence which seems to support that the model may develop a potential finite-time singularity from smooth initial data with finite energy. By exploiting the axisymmetric geometry of the problem, we obtain a very efficient adaptive solver with an optimal complexity which provides effective local resolutions of order $4096^{3}$. With this level of resolution, we obtain an excellent fit for the asymptotic blowup rate of maximum axial vorticity. If we denote by $\omega^{z}$ the axial vorticity component along the $z$-direction, we find that $\left\|\omega^{z}\right\|_{\infty}(t) \approx C(T-t)^{-1}$ and the potential singularity approaches the symmetry axis (the $z$-axis) as $t \rightarrow$ $T$. Moreover, our study seems to suggest that the potential singularity is locally self-similar and isotropic.

The initial condition considered in our numerical computations is given by

$$
\begin{aligned}
& u_{1}(z, r, 0)=(1+\sin (4 \pi z))\left(r^{2}-1\right)^{20}\left(r^{2}-1.2\right)^{30}, \\
& \psi_{1}(z, r, 0)=0, \\
& \omega_{1}(z, r, 0)=0 .
\end{aligned}
$$

A second-order finite-difference discretization is used in space, and the classical fourth-order Runge-Kutta method is used to discretize in time. Since we expect that the potential singularity will appear along the symmetry axis at $r=0$, we use the following coordinate transformation along the $r$ direction to achieve the adaptivity by clustering the grid points near $r=0$ :

$$
r=f(\alpha) \equiv \alpha-0.9 \sin (\pi \alpha) / \pi .
$$

With this change of variables, we can achieve an effective resolution up to $4096^{3}$ for the corresponding 3D problem.

We now present numerical results which show that the solution of the viscous model becomes nearly singular. We choose the viscous coefficient to be $\nu=0.001$ and perform a series of resolution studies using the adaptive method. We have used both uniform mesh and adaptive mesh with $N_{z}$ ranging from 256 to 4096 . Below we present the computational results obtained by using the adaptive mesh with the highest resolution $N_{z}=4096$, $N_{r}=400$, and $\Delta t=2.5 \times 10^{-7}$. We will also perform a resolution study to demonstrate that our computations are well resolved. 
From our analytical study of the 3D model, it follows by using a standard energy estimate that if $u_{1}$ is bounded, then the solution of the viscous $3 \mathrm{D}$ model cannot blow up in a finite time. Thus it is sufficient to monitor the growth of $\left\|u_{1}\right\|_{\infty}$ in time. We will present numerical evidence which seems to support that $u_{1}$ may develop a potential finite-time singularity for the initial condition we consider. The nature of this potential singularity and the mechanism for generating this potential singularity will be analysed in a later subsection.

In Figure 4.1, we plot the maximum of $u_{1}$ in time over the time interval $[0,0.021]$ using the adaptive mesh method with $N_{z}=4096$ and $N_{r}=400$. The time step is chosen to be $\Delta t=2.5 \times 10^{-7}$. We can see that $\left\|u_{1}\right\|_{\infty}$ experiences a very rapid growth in time after $t=0.02$. In Figure $4.1(\mathrm{~b})$, we also plot $\log \left(\log \left(\left\|u_{1}\right\|_{\infty}\right)\right)$ as a function of time. We can see clearly that $\left\|u_{1}\right\|_{\infty}$ grows much more rapidly than double exponential in time, which implies that the solution of our model may develop a finite-time singularity. We will present more careful analysis of this potentially singular behaviour later.

In Figures 4.2-4.3, we show a sequence of contour plots for $u_{1}$ from $t=0.014$ to $t=0.021$. At early times, we observe that the solution forms two large focusing centres of $u_{1}$ which approach each other. As this occurs, these rather localized regions are squeezed and form a thin layer parallel to the $r$-axis and with large gradients along the $z$-direction. As these regions approach each other and develop a thin layer parallel to the $r$-axis, the solution becomes locally $z$-dominant near the region where $u_{1}$ achieves its maximum. In this region, the $3 \mathrm{D}$ model can be approximated to the leading order by the corresponding 1D model along the $z$-direction. Hou and Lei $(2009 b)$ proved that the solution of the 1D model cannot blow up. The solution survives this potential blow-up scenario. After $t=0.0172$, the maximum of $u_{1}$ starts to decrease. The two focusing centres move away from each other and their supports become more isotropic. As time increases, we observe that there is a strong nonlinear interaction between $u_{1}$ and $\left(\psi_{1}\right)_{z}$, which is induced by the overlap between the support of maximum of $u_{1}$ and the support of maximum of $\left(\psi_{1}\right)_{z}$. By the support of maximum of $u_{1}$, we mean the region in which $u_{1}$ is comparable to its maximum. The strong alignment between $u_{1}$ and $\left(\psi_{1}\right)_{z}$ near the support of maximum of $u_{1}$ leads to a rapid growth of the solution which may become singular in a finite time.

Another important observation is that as time increases, the position at which $u_{1}$ achieves its maximum also moves towards the symmetry axis. This suggests that the potential singularity will be along the symmetry axis at the singularity time. We note that $\lim _{r \rightarrow 0+} u_{1}=0.5 \lim _{r \rightarrow 0+} \omega^{z}$. Thus, the blow-up of $u_{1}$ characterizes the blow-up of the axial vorticity, $\omega^{z}$.

Next, we perform a detailed study for the 3D model and push our computations very close to the potential singularity time. We use a sequence of 
(a)
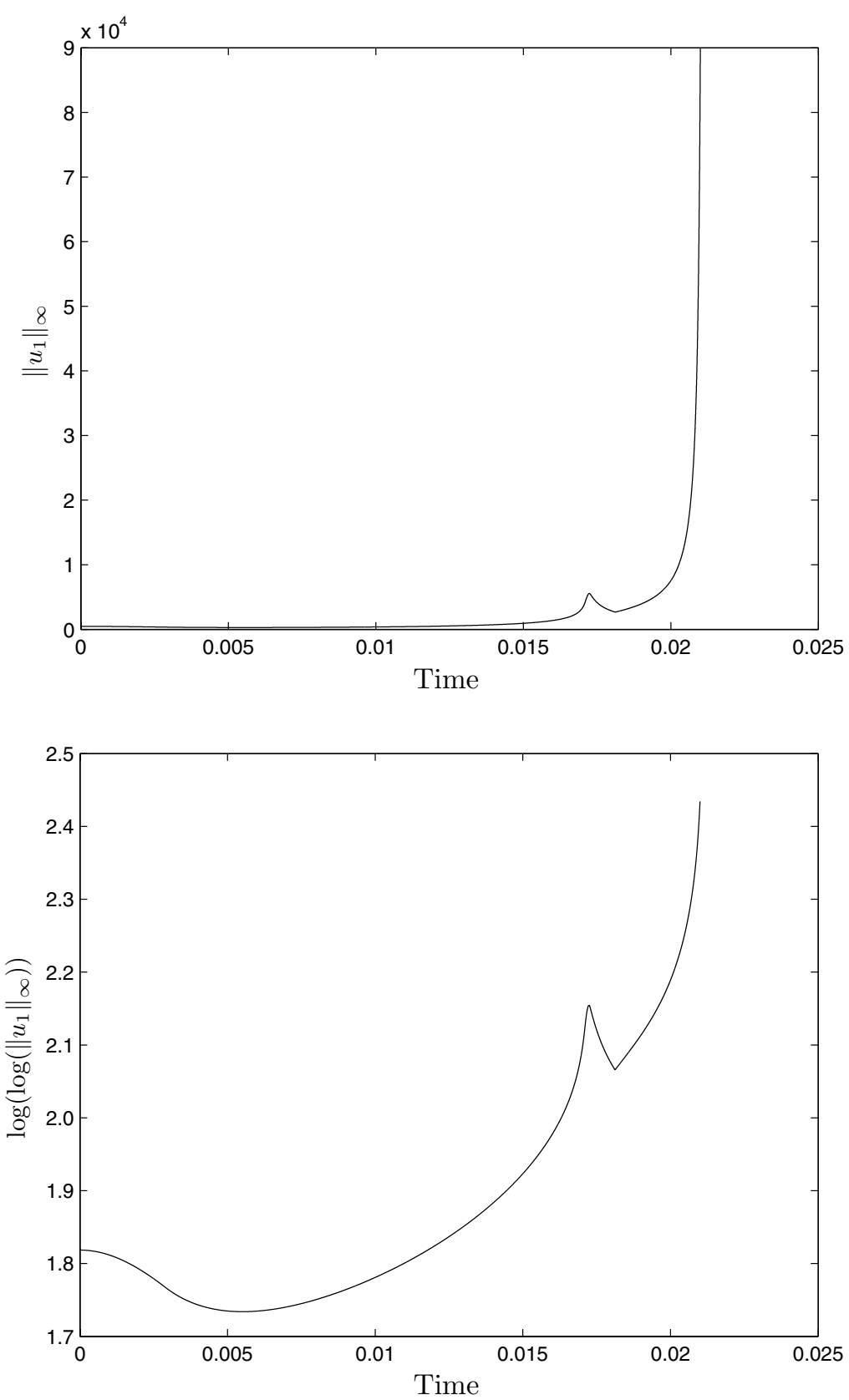

Figure 4.1. (a) $\left\|u_{1}\right\|_{\infty}$ as a function of time over the interval [0, 0.021], (b) $\log \left(\log \left(\left\|u_{1}\right\|_{\infty}\right)\right)$ as a function of time over the same interval. The solution is computed by an adaptive mesh with $N_{z}=4096, N_{r}=400, \Delta t=2.5 \times 10^{-7}$, $\nu=0.001$. 


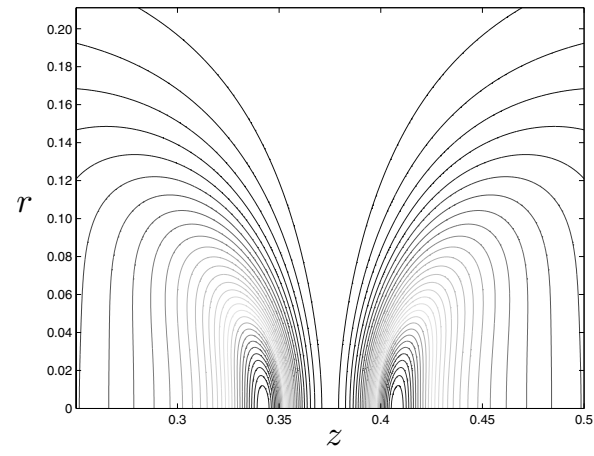

(a)

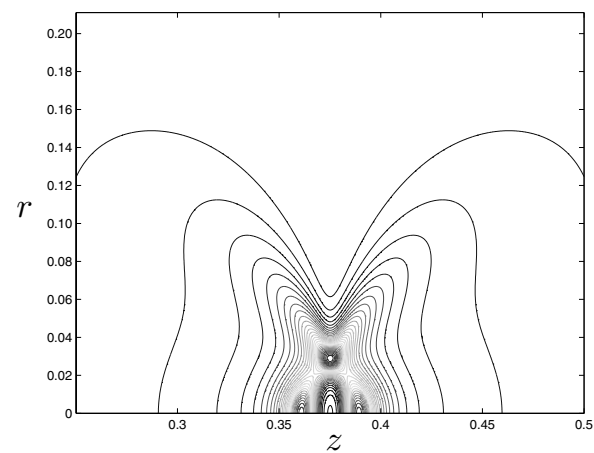

(c)

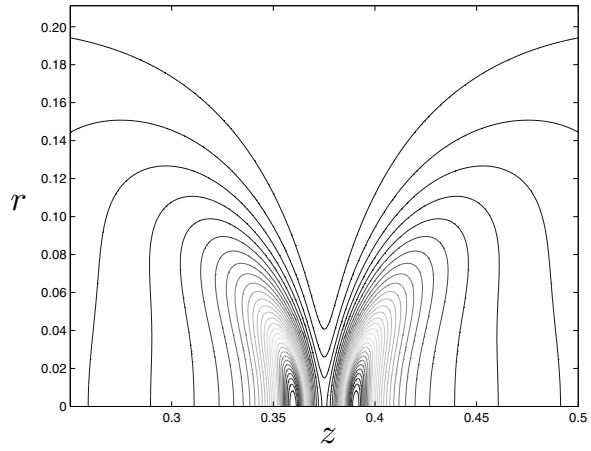

(b)

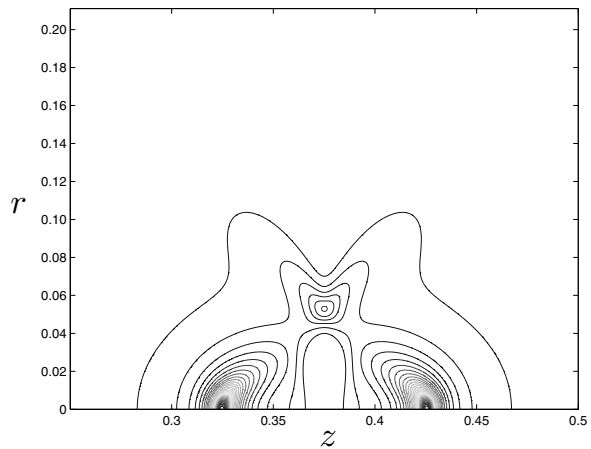

(d)

Figure 4.2. (a)-(d) The contour plots of $u_{1}$ for the viscous model at $t=0.014,0.016,0.018$ and 0.02 respectively. Adaptive mesh computation with $N_{z}=4096, N_{r}=400, \Delta t=2.5 \times 10^{-7}, \nu=0.001$.

resolutions using both uniform and adaptive mesh. For the uniform mesh, we use resolutions for $N_{z} \times N_{r}$ ranging from $256 \times 256$ to $2048 \times 2048$ with time steps ranging from $\Delta t=5 \times 10^{-6}$ to $5 \times 10^{-7}$. For the adaptive mesh, we use $N_{z} \times N_{r}=2048 \times 256, N_{z} \times N_{r}=3072 \times 328$ and $N_{z} \times N_{r}=4096 \times 400$ respectively. The corresponding time steps for these computations are $\Delta t=10^{-6}, \Delta t=5 \times 10^{-7}$, and $\Delta t=2.5 \times 10^{-7}$ respectively. With $N_{z} \times N_{r}=4096 \times 400$, we achieve an effective resolution of $4000 \times 4000$ near the region of $r=0$ where the solution is most singular.

To obtain further evidence for a potential finite-time singularity, we use a systematic singularity form fit procedure to obtain a good fit for the possible singularity of the solution. The procedure of our form fit is as 

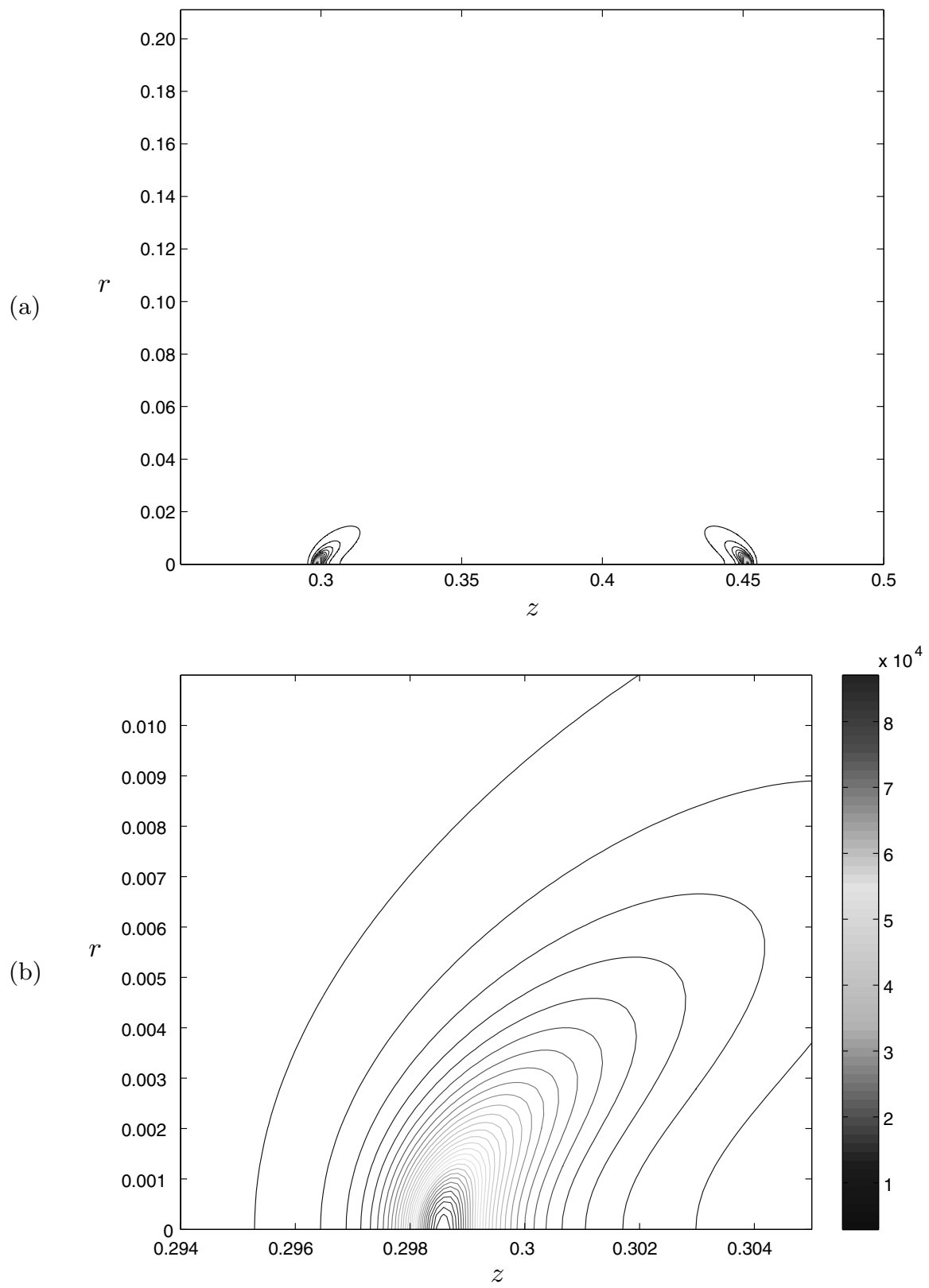

Figure 4.3. The contour of $u_{1}$ at $t=0.021$ (a) and its close-up view (b) for the viscous model computed by the adaptive mesh with $N_{z}=4096, N_{r}=400$, $\Delta t=2.5 \times 10^{-7}, \nu=0.001$. 
follows. We look for a finite-time singularity of the form

$$
\left\|u_{1}\right\|_{\infty} \approx \frac{C}{(T-t)^{\alpha}} .
$$

We have tried several ways to determine the fitting parameters $T, C$ and $\alpha$. Ultimately, we find that the best way is to study the inverse of $\left\|u_{1}\right\|_{\infty}$ as a function of time using a sequence of numerical resolutions. For each resolution, we find that the inverse of $\left\|u_{1}\right\|_{\infty}$ is almost a perfect linear function of time: see Figures 4.4 and 4.5. By using a least-squares fit of the inverse of $\left\|u_{1}\right\|_{\infty}$, we find that $\alpha=1$ gives the best fit. The same least-squares fit also determines the potential singularity time $T$ and the constant $C$. We remark that the $O(1 /(T-t))$ blow-up rate of $u_{1}$, which measures the axial vorticity, is consistent with the non-blow-up criterion of Beale-Kato-Majda type.

To confirm that the above procedure indeed gives a good fit for the potential singularity, we plot $\left\|u_{1}\right\|_{\infty}^{-1}$ as a function of time in Figure $4.4(\mathrm{a})$. We can see that the agreement between the computed solution with $N_{z} \times N_{r}=$ $4096 \times 400$ and the fitted solution is almost perfect. In Figure 4.4(b) we plot $\left\|u_{1}\right\|_{\infty}$ computed by our adaptive method against the form fit $C /(T-t)$ with $T=0.02109$ and $C=8.20348$. The two curves are almost indistinguishable during the final stage of the computation from $t=0.018$ to $t=0.021$.

We further investigate the potential singular behaviour of the solution by using a sequence of resolutions to study the limiting behaviour of the

Table 4.1. Resolution study of parameters $T$ and $C$ in the asymptotic fit for the viscous model: $\left\|u_{1}\right\|_{\infty}^{-1} \approx \frac{(T-t)}{C}$ using different resolutions $h_{z}=1 /\left(2 N_{z}\right)$. The resolutions we use in our adaptive computations are $N_{z} \times N_{r}=1024 \times 128,2048 \times 256,3072 \times 328$ and $4096 \times 400$ respectively. The corresponding time steps are $\Delta t=10^{-6}, 5 \times 10^{-7}$, $3.625 \times 10^{-7}$ and $2.5 \times 10^{-7}$ respectively. The last row is obtained by extrapolating the second-order polynomial that interpolates the data obtained using $h_{z}=1 / 4096,1 / 6144$ and $1 / 8192$.

\begin{tabular}{c|l|l}
\hline \hline \multicolumn{1}{c}{$h_{z}$} & \multicolumn{1}{|c}{$T$} & $C$ \\
\hline $1 / 2048$ & 0.02114 & 8.409 \\
$1 / 4096$ & 0.0211 & 8.2237 \\
$1 / 6144$ & 0.021093 & 8.20946 \\
$1 / 8192$ & 0.02109 & 8.20348 \\
extrapolation to $h_{z}=0$ & 0.021083 & 8.1901 \\
\hline
\end{tabular}


(a)
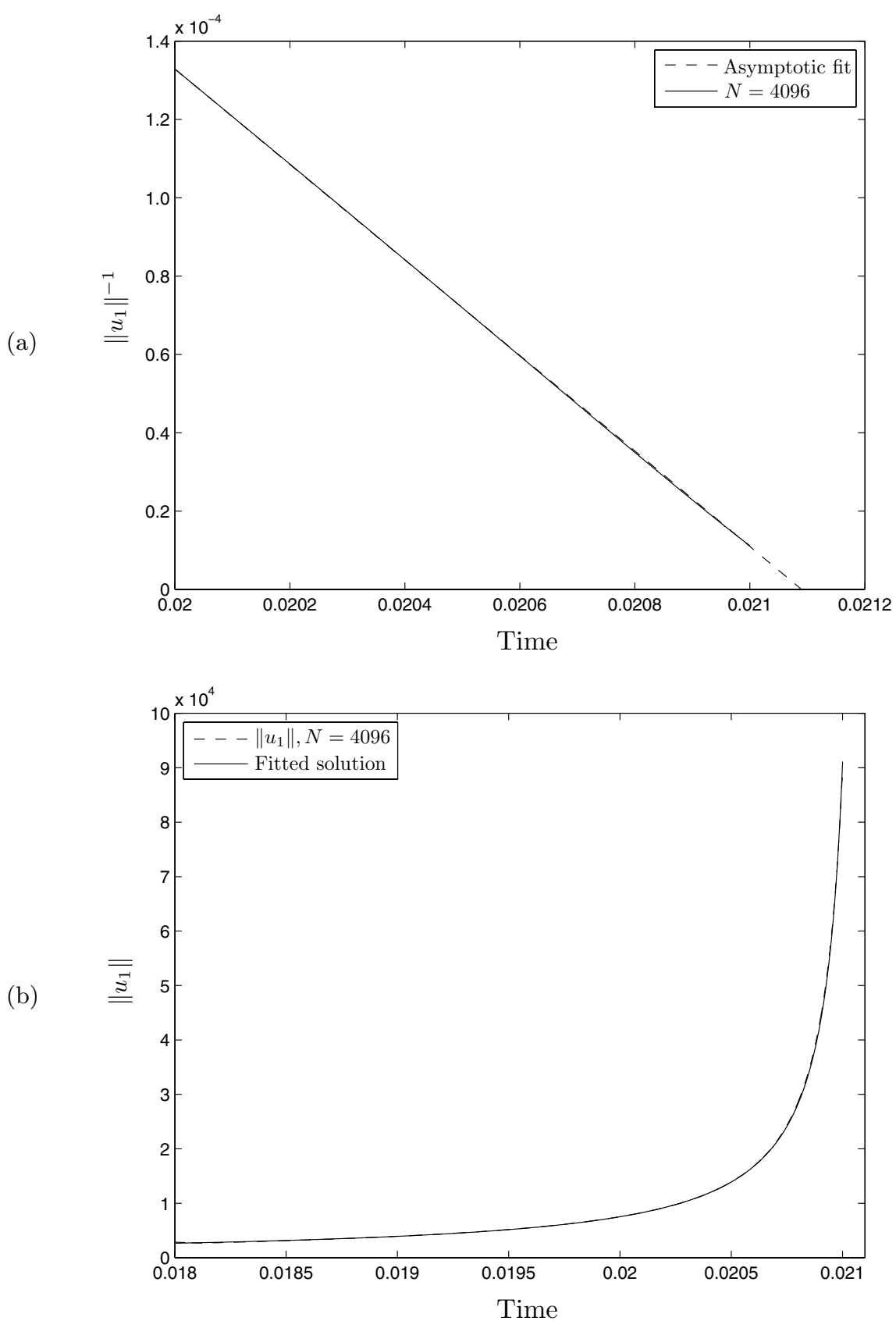

Figure 4.4. (a) The inverse of $\left\|u_{1}\right\|_{\infty}$ (solid line) versus the asymptotic fit (dashed line) for the viscous model; (b) $\left\|u_{1}\right\|_{\infty}$ (solid line) versus the asymptotic fit (dashed line). The asymptotic fit is of the form $\left\|u_{1}\right\|_{\infty}^{-1} \approx \frac{(T-t)}{C}$ with $T=0.02109$ and $C=8.20348$. The solution is computed by adaptive mesh with $N_{z}=4096, N_{r}=400, \Delta t=2.5 \times 10^{-7} ; \nu=0.001$. 


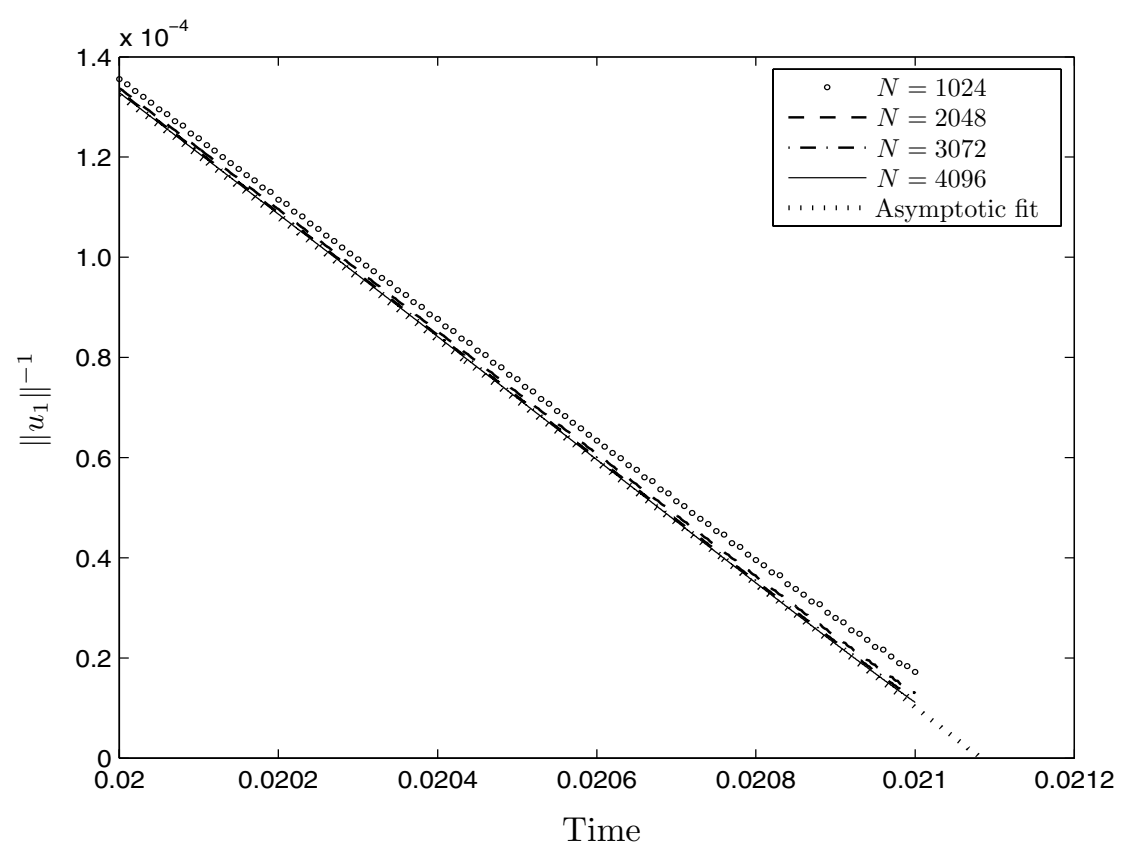

Figure 4.5. The inverse of $\left\|u_{1}\right\|_{\infty}$ in time for the viscous model. The solution is computed by adaptive mesh with $N_{z}=1024$, 2048, 3072 and 4096 respectively (ordering from top to bottom in the figure), $\Delta t=10^{-6}, 5 \times 10^{-7}, 3.625 \times 10^{-7}$, and $2.5 \times 10^{-7}$ respectively.

The last curve is the singularity fit by extrapolating the computational results obtained by $N_{z}=2048,3072$ and 4096 to infinite resolution $N_{z}=\infty$. The fitted curve is of the form $\left\|u_{1}\right\|_{\infty}^{-1} \approx(T-t) / C$, with $T=0.021083$ and $C=8.1901 ; \nu=0.001$.

computed solution as we refine our resolutions. The space resolutions we use are $N_{z} \times N_{r}=1024 \times 128,2048 \times 256,3072 \times 328$ and $4096 \times 400$ respectively. The corresponding time steps are $\Delta t=10^{-6}, 5 \times 10^{-7}, 3.625 \times 10^{-7}$ and $2.5 \times 10^{-7}$ respectively. For each resolution, we obtain an optimal leastsquares fit of the singularity of the form $\left\|u_{1}\right\|_{\infty}^{-1} \approx(T-t) / C$. The results are summarized in Table 4.1. Based on the fitted parameters $T$ and $C$ from the three largest resolutions, we construct a second-order polynomial that interpolates $T$ and $C$ through these three data points. We then use the polynomial to extrapolate the values of $T$ and $C$ to the infinite resolution limit. The extrapolated values at $h_{z}=0$ are $T=0.021083$ and $C=8.1901$ respectively. In Figure 4.5, we plot the inverse of $\left\|u_{1}\right\|_{\infty}$ as a function of time using four different resolutions. We can see that as we refine the resolution, the computed solution converges to the extrapolated singularity limiting profile. 


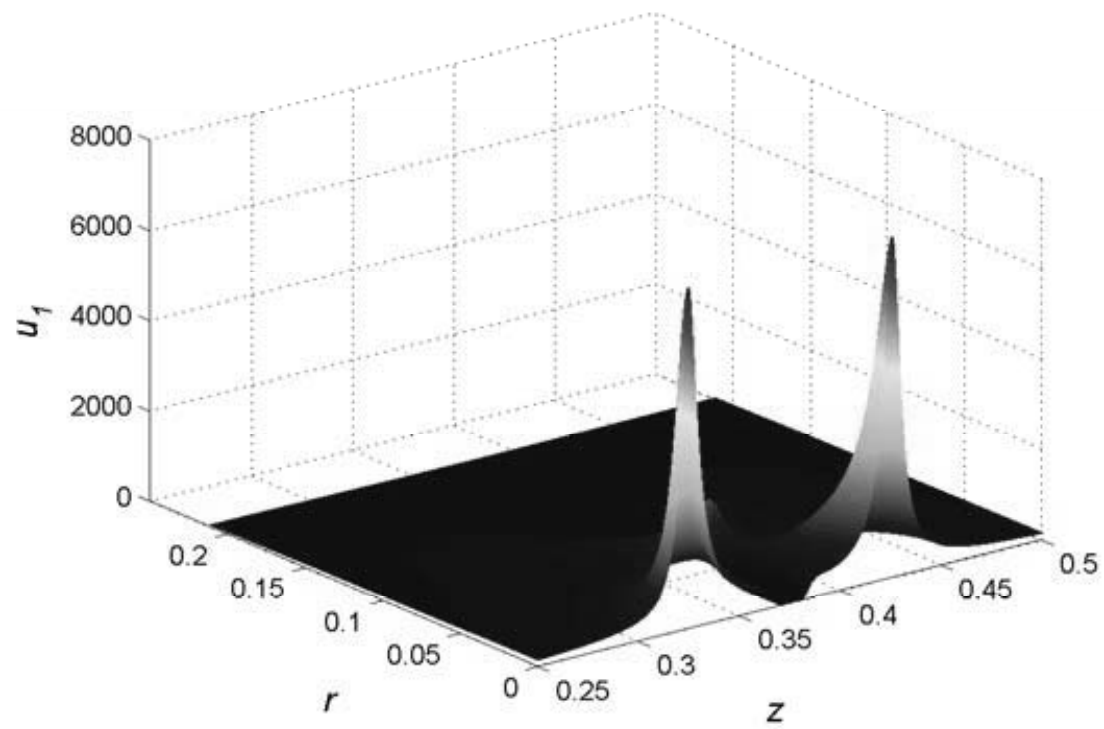

Figure 4.6. The 3D view of $u_{1}$ at $t=0.02$ for the viscous model computed by the adaptive mesh with $N_{z}=4096, N_{r}=400$, $\Delta t=2.5 \times 10^{-7}, \nu=0.001$.

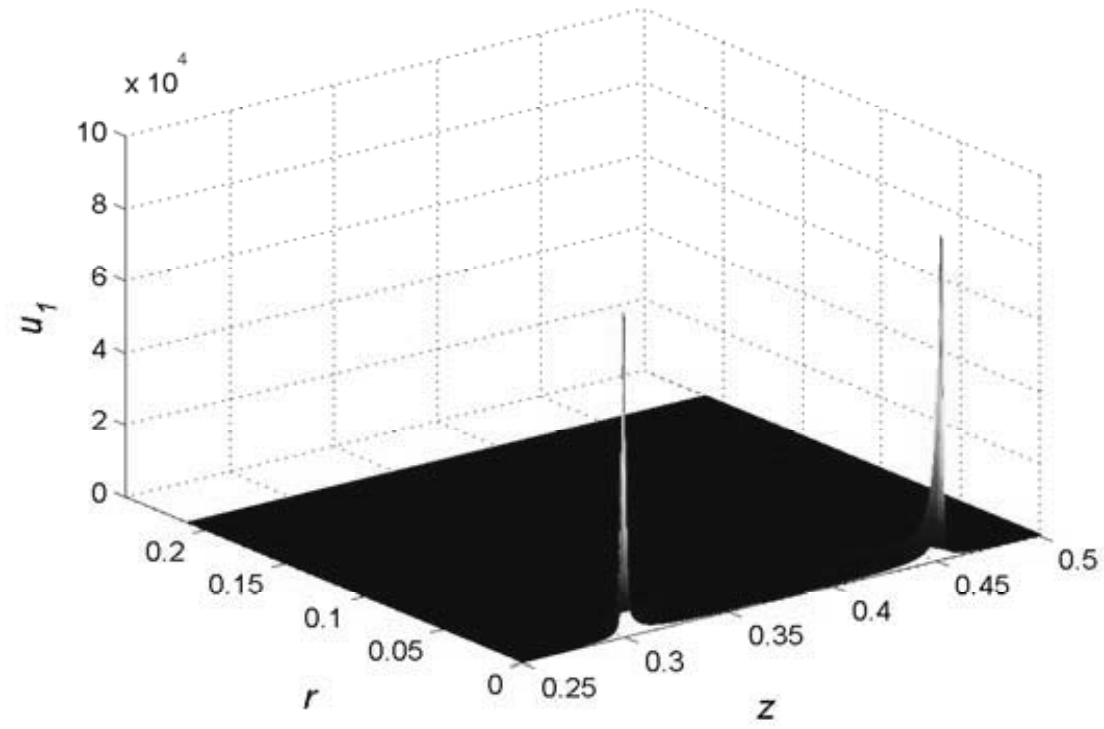

Figure 4.7. The $3 \mathrm{D}$ view of $u_{1}$ at $t=0.021$ for the viscous model computed by the adaptive mesh with $N_{z}=4096, N_{r}=400$, $\Delta t=2.5 \times 10^{-7}, \nu=0.001$. 


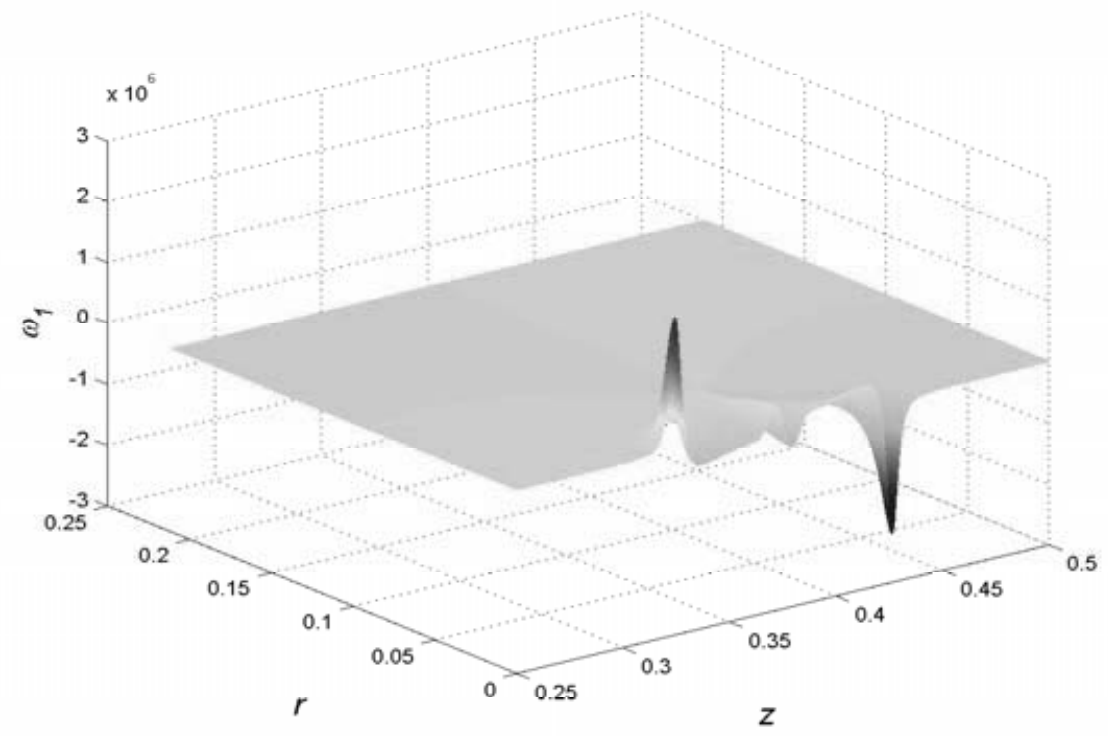

Figure 4.8. The $3 \mathrm{D}$ view of $\omega_{1}$ at $t=0.02$ for the viscous model computed by the adaptive mesh with $N_{z}=4096, N_{r}=400$, $\Delta t=2.5 \times 10^{-7}, \nu=0.001$.

To illustrate the nature of the nearly singular solution, we show the 3D view of $u_{1}$ as a function of $r$ and $z$ in Figures 4.6 and 4.7. We also show the $3 \mathrm{D}$ view of $w_{1}$ as a function of $r$ and $z$ in Figure 4.8. While $u_{1}$ is symmetric with respect to $z=0.375, w_{1}$ is anti-symmetric with respect to $z=0.375$. We can see that the support of the solution $u_{1}$ in the most singular region is isotropic and appears to be locally self-similar (Hou and Lei 2009b).

\section{Resolution study}

Finally, we perform a resolution study for our computations by comparing the computation obtained by three different resolutions, which are $N_{z} \times$ $N_{r}=2048 \times 256, N_{z} \times N_{r}=3072 \times 328$, and $N_{z} \times N_{r}=4096 \times 400$. In Figure 4.9, we plot $\left\|u_{1}\right\|_{\infty}$ as a function of time using these three resolutions $N_{z} \times N_{r}=2048 \times 256, N_{z} \times N_{r}=3072 \times 328$, and $N_{z} \times N_{r}=4096 \times 400$ over the time interval $[0,0.021]$. We can see that while the computation with $N_{z}=2048$ under-resolves the solution near the end of the computation, the solution obtained by using $N_{z}=3072$ gives an excellent agreement with that obtained by using $N_{z}=4096$.

We also compare the solution of $u_{1}$ at $r=0$ using three different resolutions. Using the partial regularity theory for the 3D model, any singularity of our 3D model must lie on the symmetry axis, $r=0$. Thus it makes 
(a)

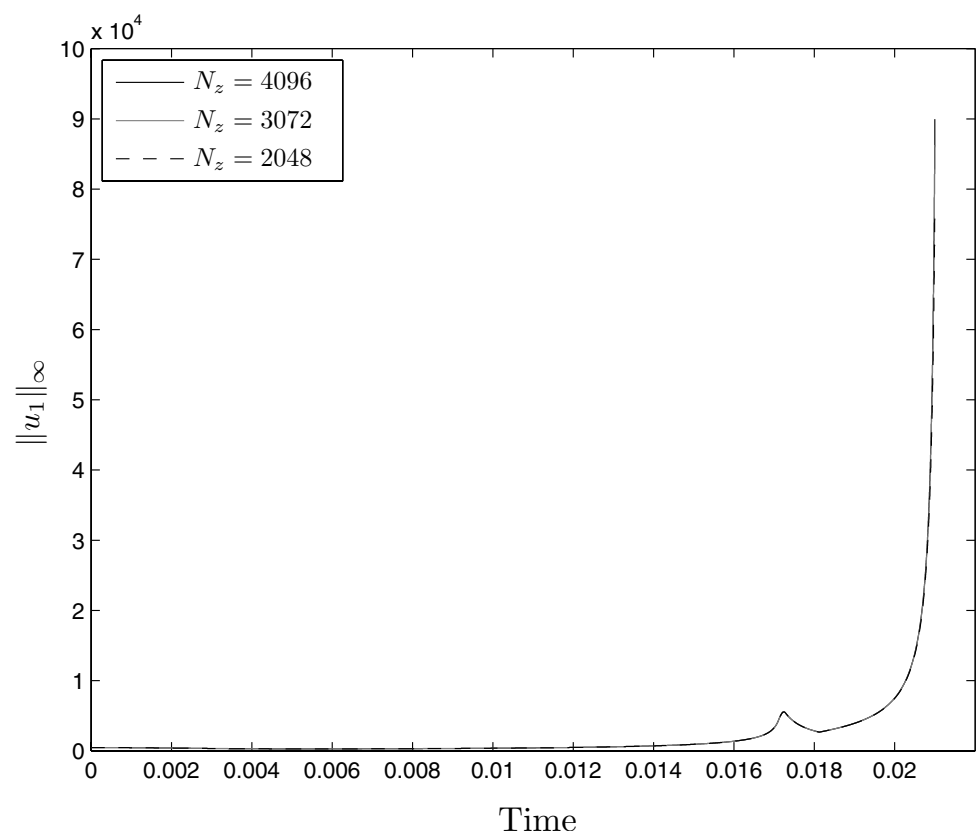

(b)

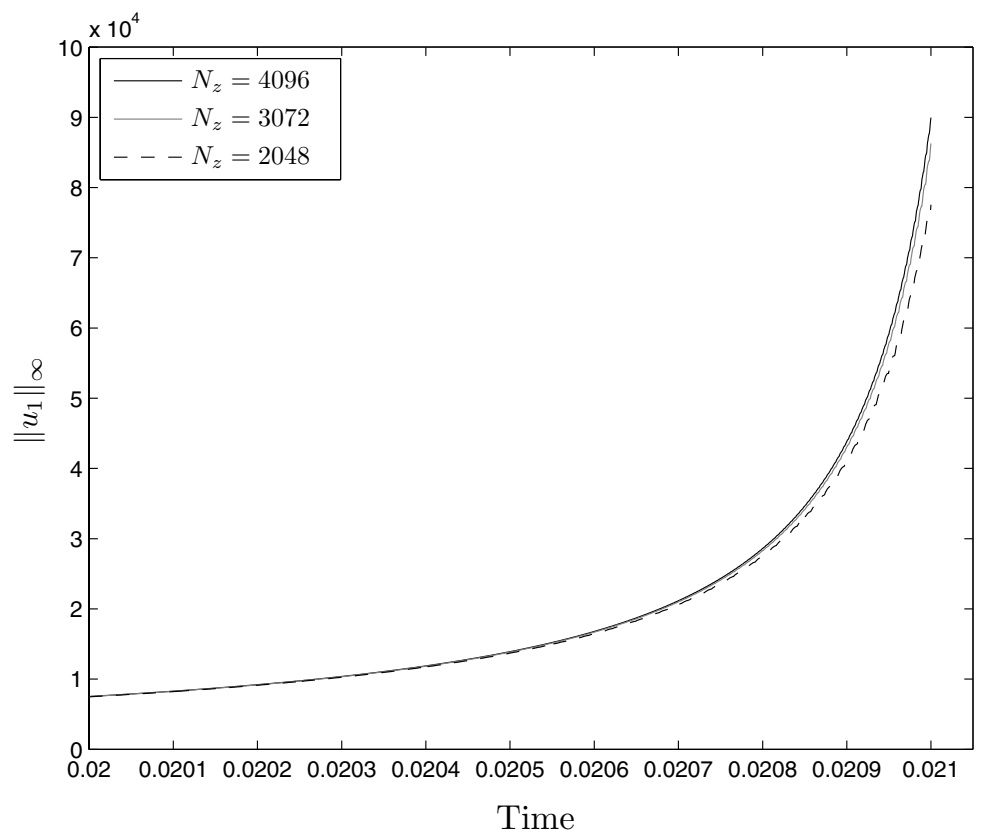

Figure 4.9. Convergence study for $\left\|u_{1}\right\|_{\infty}$ in time for the viscous model with three resolutions: $N_{z} \times N_{r}=2048 \times 256, \Delta t=5 \times 10^{-7}$ (dashed line), $N_{z} \times N_{r}=3072 \times 328, \Delta t=3.625 \times 10^{-7}$ (grey line), $N_{z} \times N_{r}=4096 \times 400$, $\Delta t=2.5 \times 10^{-7}$ (solid line). Figure (a) is over the time interval [0, 0.021]; (b) is a close-up view over the time interval [0.02, 0.021]; $\nu=0.001$. 
(a)
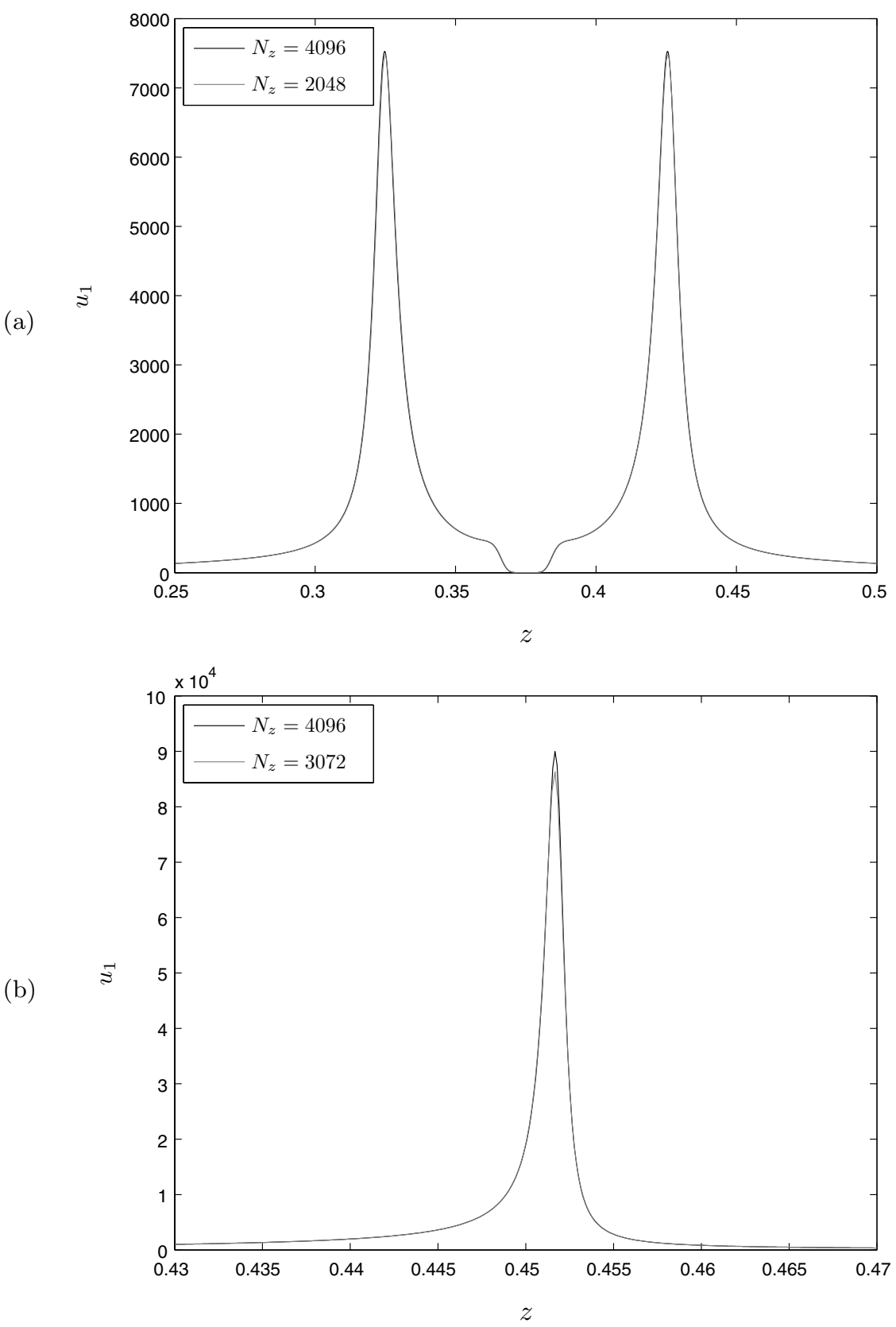

Figure 4.10. Convergence study for $u_{1}$ at $r=0$ and $t=0.02$ and $t=0.021$ for the viscous model with different resolutions. Figure (a) is the comparison between $N_{z} \times N_{r}=2048 \times 256$ (solid line) and $N_{z} \times N_{r}=4096 \times 400$ (grey line); (b) is the comparison between $N_{z} \times N_{r}=3072 \times 328$ (solid line) and $N_{z} \times N_{r}=4096 \times 400$ (grey line); $\nu=0.001$. 
sense to perform a resolution study for the solution along the symmetry axis which is the most singular region of the solution. In Figure 4.10(a), we plot the solutions obtained by two resolutions using $N_{z} \times N_{r}=2048 \times 256$ $\left(\Delta t=5 \times 10^{-7}\right)$ and $N_{z} \times N_{r}=4096 \times 400\left(\Delta t=2.5 \times 10^{-7}\right)$ on top of each other at $t=0.02$. The two solutions are almost indistinguishable. However, the computation with $N_{z} \times N_{r}=2048 \times 256$ is not sufficient to resolve the nearly singular behaviour of the solution at $t=0.021$. On the other hand, the computation with $N_{z} \times N_{r}=3072 \times 328\left(\Delta t=3.625 \times 10^{-7}\right)$ gives much improved resolution. In Figure 4.10(b) we compare the solution obtained by using $N_{z} \times N_{r}=3072 \times 328$ with that obtained by using $N_{z} \times N_{r}=4096 \times 400$ at $t=0.021$. We observe that the agreement of the two solutions is very good except near the points where $u_{1}$ attains its maximum.

\subsection{Mechanism for a finite-time blow-up}

To understand the mechanism for the potential blow-up of the viscous model, we plot the solution $u_{1}$ on top of $\left(\psi_{1}\right)_{z}$ along the symmetry axis $r=0$ at $\mathrm{t}=0.021$ in Figure 4.11. We see that there is a significant overlap between the supports of the maximum of $u_{1}$ and of the maximum of $\left(\psi_{1}\right)_{z}$. Moreover, the solution $u_{1}$ has a strong alignment with $\left(\psi_{1}\right)_{z}$ near the region of maximum of $u_{1}$. The local alignment between $u_{1}$ and $\left(\psi_{1}\right)_{z}$ induces a strong nonlinearity on the right-hand side of the $u_{1}$-equation, which has the form $2\left(\psi_{1}\right)_{z} u_{1}$. This strong alignment between $u_{1}$ and $\left(\psi_{1}\right)_{z}$ is the main mechanism for the potential finite-time blow-up of the 3D model. Similar alignment between $u_{1}$ and $\left(\psi_{1}\right)_{z}$ near the region of maximum $u_{1}$ is also observed for the inviscid model (Hou and Lei 2009b).

It is interesting to note that the position at which $u_{1}$ attains its maximum does not coincide with that at which $\left(\psi_{1}\right)_{z}$ attains its maximum. In fact, at the point where $u_{1}$ reaches its maximum, the value of $\left(\psi_{1}\right)_{z}$ is relatively small, or even negative. This misalignment between the position at which $u_{1}$ attains its maximum and the position at which $\left(\psi_{1}\right)_{z}$ attains its maximum induces a dynamic motion which pushes the two focusing centres of $u_{1}$ to move away from each other. This dynamics reinforces the local alignment between $u_{1}$ and $\left(\psi_{1}\right)_{z}$. We remark that this wave-like behaviour of the solution along the $z$-direction is consistent with the nonlinear non-local wave equation (4.10) that we derived for $v=\log \left(u_{1}^{2}\right)$ for the inviscid model.

As we see in the next subsection, the inclusion of the convection term forces the two focusing centres to travel towards each other. Moreover, the local alignment between $u_{1}$ and $\left(\psi_{1}\right)_{z}$ is destroyed. As a result, the solution becomes defocused and smoother along the symmetry axis. There is no evidence that the solution of the full Navier-Stokes equations would develop a finite-time singularity, at least for the time interval considered here. 
(a)

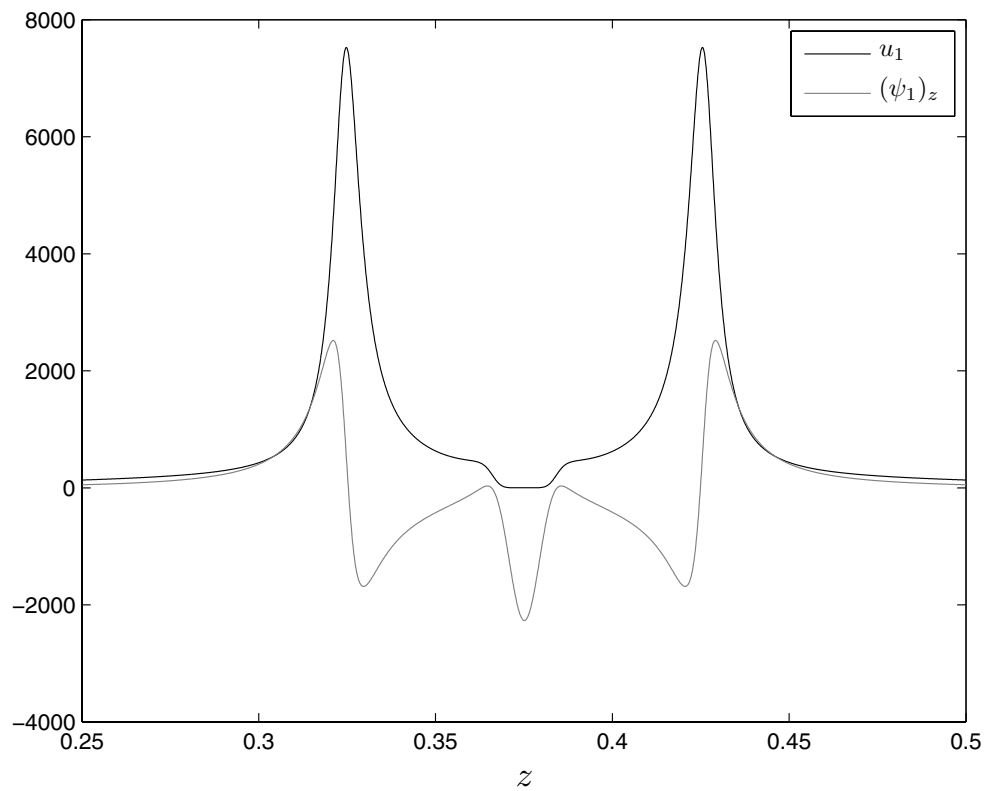

(b)

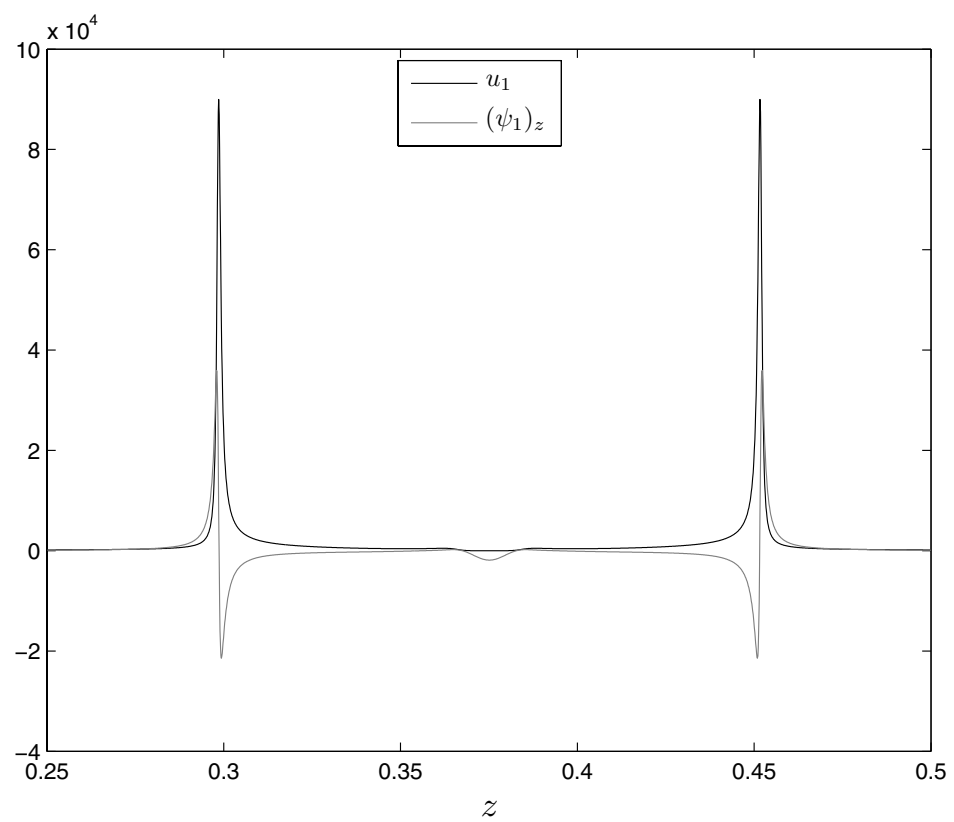

Figure 4.11. $u_{1}$ (solid line) versus $\left(\psi_{1}\right)_{z}$ (grey line) of the viscous model along the symmetry axis $r=0$. (a) $t=0.02$; (b) $t=0.021$. Adaptive mesh computation with $N_{z}=4096, N_{r}=400, \Delta t=2.5 \times 10^{-7}, \nu=0.001$. 


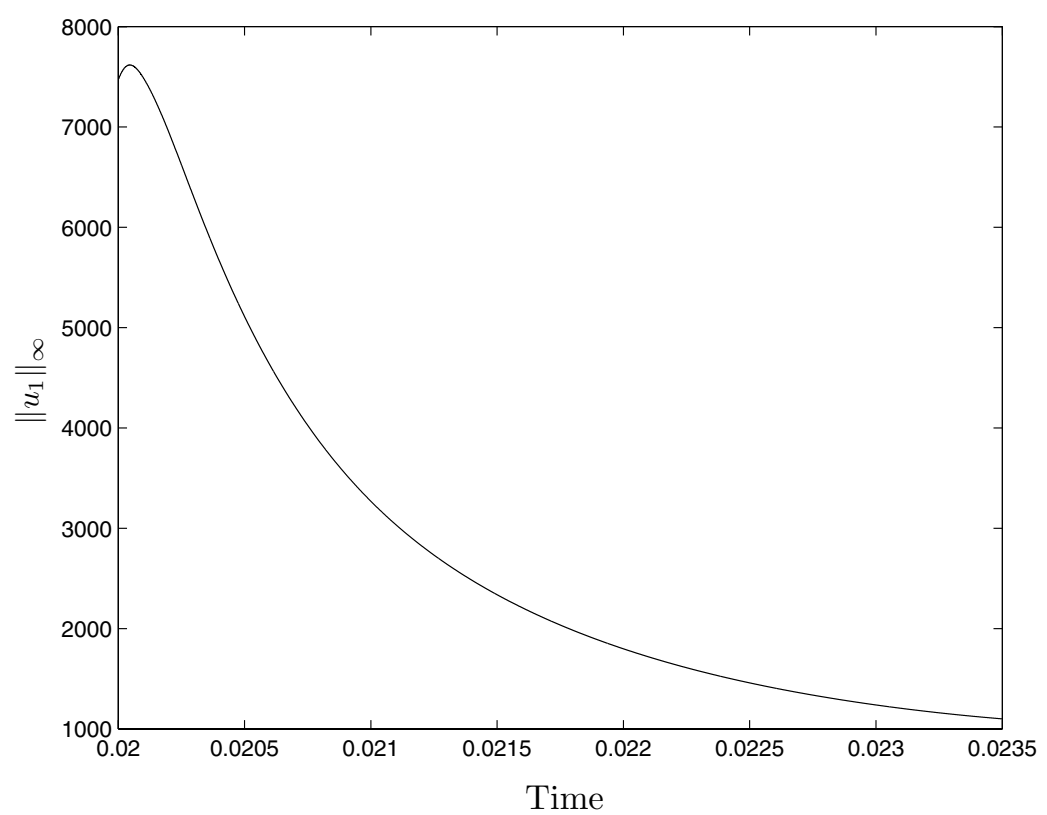

Figure 4.12. $\left\|u_{1}\right\|_{\infty}$ in time, the full Navier-Stokes computation using the solution of the $3 \mathrm{D}$ viscous model at $t=0.02$ as the initial condition. Adaptive mesh computation with $N_{z}=2048, N_{r}=1024, \nu=0.001$.

\subsection{The stabilizing effect of the convection term}

In this subsection, we show that by adding back the convection term to the 3D model, which recovers the reformulated Navier-Stokes equations, the solution behaves completely differently. The mechanism for generating the potential finite time singularity for the 3D model is destroyed. Even if we start with the nearly singular solution obtained by the 3D model at $t=0.02$ and use it as the initial condition for the full Navier-Stokes equations, we observe that the maximum of $u_{1}$ soon decreases in time: see Figure 4.12. It is easy to see that the 3D axisymmetric Navier-Stokes equations with swirl cannot develop a finite-time singularity if $u_{1}$ is bounded. Thus the fact that $\left\|u_{1}\right\|_{\infty}$ is decreasing in time is a clear indication that the solution does not develop a finite-time singularity, at least over the time interval considered here.

We also observe that the local alignment between $u_{1}$ and $\left(\psi_{1}\right)_{z}$ near the region of maximum $u_{1}$ is destroyed by including the convection term (see Figure 4.13), as is the focusing mechanism. The solution becomes defocused (see Figure 4.14). As time evolves, the two focusing centres approach each other. This process creates a strong internal layer orthogonal to the $z$-axis 
(a)

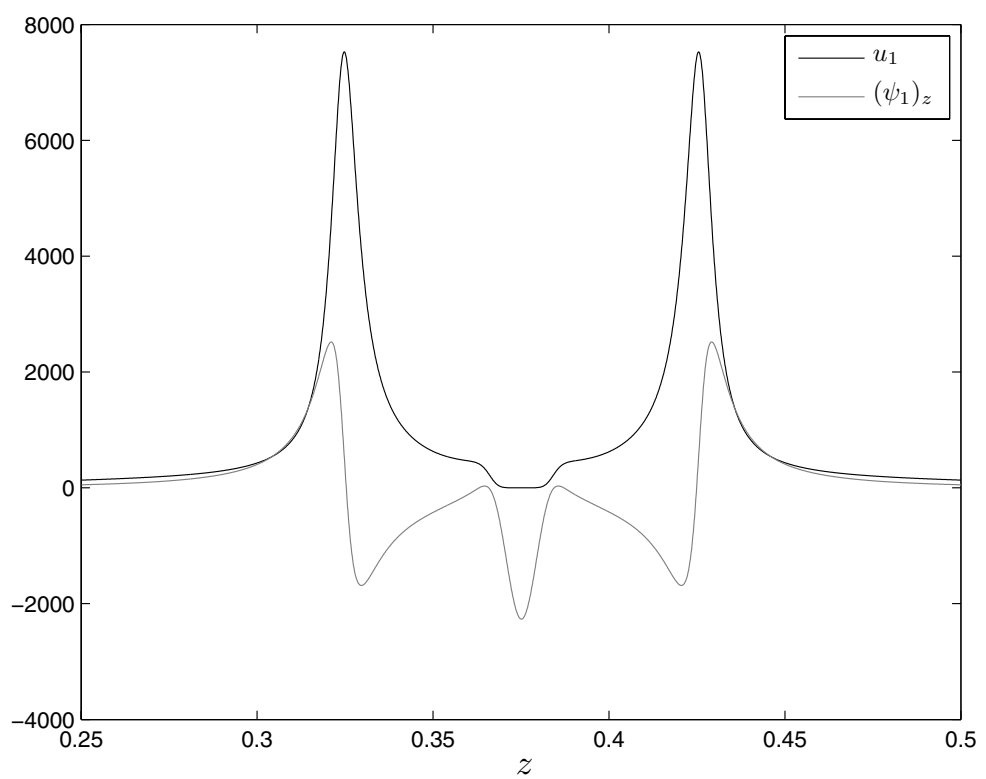

(b)

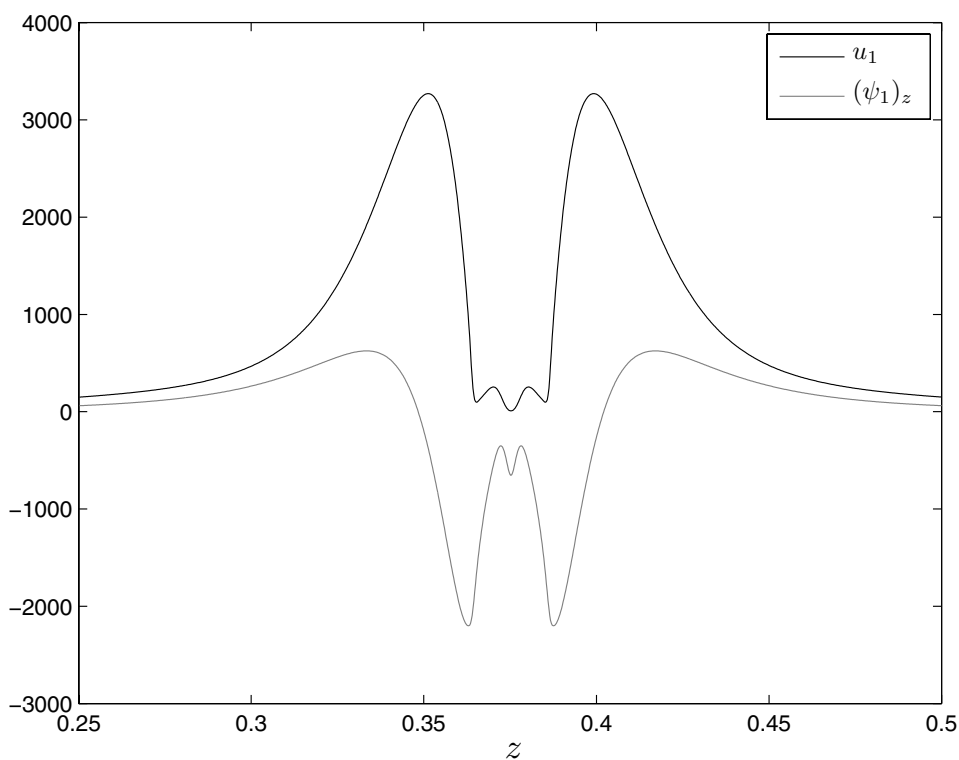

Figure 4.13. $u_{1}$ (solid line) versus $\left(\psi_{1}\right)_{z}$ (grey line) along the symmetry axis $r=0$. Figure (a) corresponds to $t=0.02$ (the solution from the $3 \mathrm{D}$ viscous model); (b) corresponds to $t=0.021$ obtained by solving the full Navier-Stokes equations. Adaptive mesh computation with $N_{z}=2048, N_{r}=1024, \nu=0.001$. 


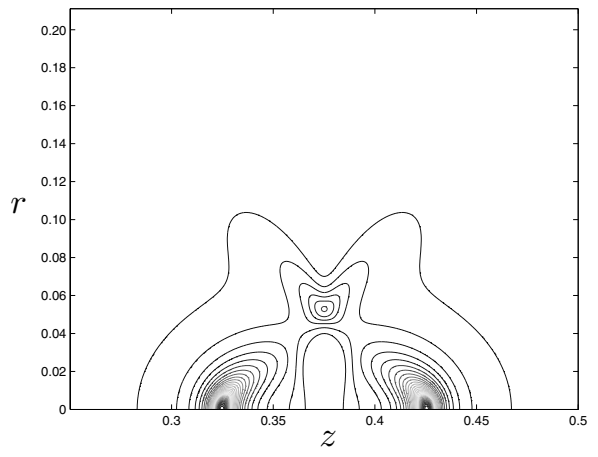

(a)

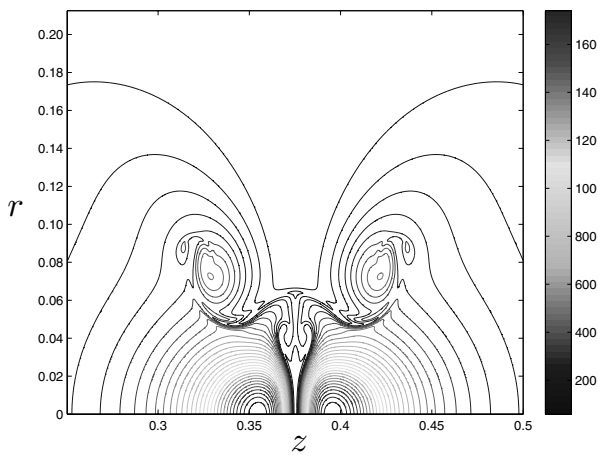

(c)

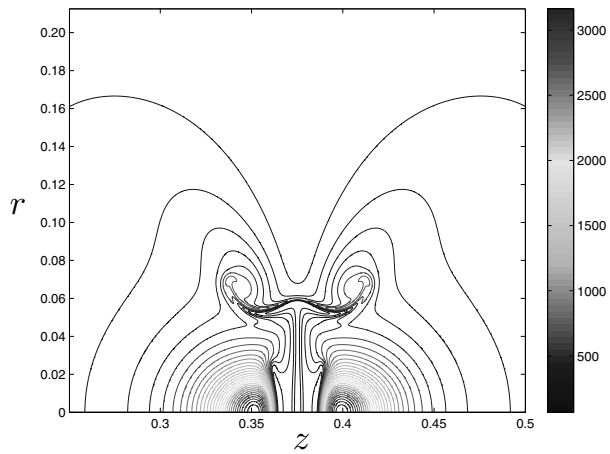

(b)

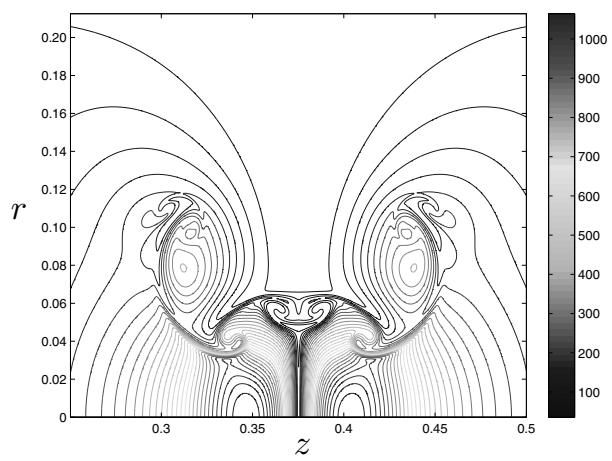

(d)

Figure 4.14. (a) The contour of $u_{1}$ at $t=0.02$ obtained from the $3 \mathrm{D}$ viscous model which serves as the initial condition for the full Navier-Stokes equations. (b) The contour of $u_{1}$ at $t=0.021$ obtained by solving the full Navier-Stokes equations. (c), (d) The contours of $u_{1}$ at $t=0.022$ and $t=0.0235$, respectively, by solving the full Navier-Stokes equations. Adaptive mesh computation with $N_{z}=2048$, $N_{r}=1024, \nu=0.001$.

and forms a jet that moves away from the symmetry axis (the $z$-axis). The jet further generates some interesting vortex structures. This is illustrated in Figure 4.14. Since the most singular part of the solution of the NavierStokes equations moves away from the symmetry axis, we use a higherresolution adaptive mesh along the $r$-direction with $N_{r}=1024$ to better resolve the layered structure along the $r$-axis.

By the Caffarelli-Kohn-Nirenberg theory, the singularity of the 3D axisymmetric Navier-Stokes equations, if there is any, must be along the sym- 
metry axis. The fact that the most singular part of the solution moves away from the symmetry axis indicates that the full Navier-Stokes equations will not form a finite-time singularity, at least not for the initial condition we consider here over the time interval for which we compute the solution. On the other hand, the solution of the 3D model with the same initial condition seems to develop a potential finite-time singularity in an earlier time. This confirms that convection plays an essential role in depleting the destabilizing effect induced by vortex stretching.

\section{Concluding remarks}

Our analysis and computations revealed a subtle dynamic depletion of vortex stretching. Sufficient numerical resolution is essential to capture this dynamic depletion. Our computations for the two antiparallel vortex tubes initial data showed that the velocity is bounded and that the vortex stretching term is bounded by $C\|\omega\|_{L^{\infty}} \log \left(\|\omega\|_{L^{\infty}}\right)$. In Hou and Li (2008b), we also repeated the computation of $\mathrm{R}$. Pelz using highly symmetric initial data (Pelz 1997). We found that while Pelz's vortex filament model indeed produces a finite-time self-similar singularity, the solution of the full 3D Euler equation with the same initial data gives only very modest growth dynamically. No evidence of finite-time singularities was found. Pelz's vortex filament computation was inspired by his earlier computation of the 3D Navier-Stokes equations (Boratav and Pelz 1994). However, our computation showed that the rapid growth of vorticity observed by Boratav and Pelz (1994) was due to under-resolution of his numerical solution (Hou and $\mathrm{Li} 2008 b)$. The actual growth of maximum vorticity was only exponential in the time interval when the solution was still well resolved. It is natural to ask if the dynamic depletion that we observed is generic, and to consider the driving mechanism for this depletion of vortex stretching. Some recent progress has been made in analysing the dynamic depletion of vortex stretching and nonlinear stability for 3D axisymmetric flows with swirl (Hou and Li 2008a, Hou, Lei and Li 2008). A related study for the 2D quasi-geostrophic model can be also found in Deng, Hou, Li and Yu (2006b). The local geometric structure of the solution near the region of maximum vorticity and the anisotropic scaling of the support of maximum vorticity seem to play a key role in the dynamic depletion of vortex stretching.

We also studied the dynamic stability of the 3D Navier-Stokes equations via an exact $1 \mathrm{D}$ model. This $1 \mathrm{D}$ model is an exact reduction of the $3 \mathrm{D}$ Navier-Stokes equations along the symmetry axis for a special class of initial data. It retains some essential nonlinear features of the 3D Navier-Stokes equations. We proved the global regularity of this $1 \mathrm{D}$ model by using a pointwise estimate. The key was to show that a positive Lyapunov function 
satisfies a new maximum principle. Here convection played an essential role in cancelling the destabilizing vortex stretching terms. Using the solution of the 1D model as a building block, we constructed a family of solutions of the 3D Navier-Stokes equations which experience interesting dynamic growth but remain smooth for all times.

To gain further understanding of the stabilizing effect of convection, we constructed a new 3D model by neglecting the convection term from the reformulated Navier-Stokes equations. This 3D model shares almost all properties of the Navier-Stokes equations, including an equivalent energy identity and a partial regularity result. Our numerical results seemed to support the conclusion that the solution of the 3D model develops locally self-similar isotropic singularities. But when we added the convection term back to the 3D model, the mechanism for generating the finite-time singularity in the 3D model was destroyed.

The results presented in this paper may have some important implication to the global regularity of the 3D Navier-Stokes equations. Our studies indicate that a successful strategy in analysing the global regularity of the 3D Navier-Stokes equations need to take advantage of the stabilizing effect of the convection term in an essential way. So far most of the regularity analysis for the 3D Navier-Stokes equations has not used the stabilizing effect of the convection term. In many cases, the same results can also be obtained for our 3D model. We are currently working to prove that the 3D model develops finite-time singularities from smooth initial data with finite energy. Such a theoretical result would show convincingly that traditional energy estimates are inadequate to prove global regularity of the 3D Navier-Stokes equations. New analytical tools that exploit the local geometric structure of the solution and the stabilizing effect of convection would be needed.

We also investigated the performance of pseudo-spectral methods in computing nearly singular solutions of fluid dynamics equations. In particular, we proposed a novel pseudo-spectral method with a high (36th)-order Fourier smoothing which retains a significant portion of the Fourier modes beyond the $2 / 3$ cut-off point. We demonstrated that the pseudo-spectral method with the high-order Fourier smoothing gives a much better performance than the pseudo-spectral method with the $2 / 3$ de-aliasing rule. Moreover, we showed that the high-order Fourier smoothing method captures about $12 \sim 15 \%$ more effective Fourier modes in each dimension than the $2 / 3$ de-aliasing method. For the 3D Euler equations, the gain in the effective Fourier codes for the high-order Fourier smoothing method can be as large as $20 \%$ over the $2 / 3$ de-aliasing method. Another interesting observation was that the error produced by the high-order Fourier smoothing method is highly localized near the region where the solution is most singular, while the $2 / 3$ de-aliasing method tends to produce oscillations in 
the entire domain. The high-order Fourier smoothing method was found be very stable dynamically. No high-frequency instability was observed.

\section{Acknowledgements}

I would like to thank my collaborators, Drs Jian Deng, Zhen Lei, Congming $\mathrm{Li}$, Ruo $\mathrm{Li}$, and Xinwei $\mathrm{Yu}$, who have contributed significantly to the results presented in this survey paper. We would like to thank Prof. Lin-Bo Zhang from the Institute of Computational Mathematics in Chinese Academy of Sciences (CAS) for providing us with the computing resources to perform this large-scale computational project. Additional computing resources were provided by the Center of High Performance Computing in CAS. We also thank Prof. Robert Kerr for providing us with his Fortran subroutine generating his initial data. This work was in part supported by the NSF under the NSF grant DMS-0713670.

\section{REFERENCES}

A. Babin, A. Mahalov and B. Nicolaenko (2001), '3D Navier-Stokes and Euler equations with initial data characterized by uniformly large vorticity', Indiana Univ. Math. J. 50, 1-35.

J. T. Beale, T. Kato and A. Majda (1984), 'Remarks on the breakdown of smooth solutions of the 3-D Euler equations', Comm. Math. Phys. 96, 61-66.

O. N. Boratav and R. B. Pelz (1994), 'Direct numerical simulation of transition to turbulence from a high-symmetry initial condition', Phys. Fluids 6, 27572784 .

J. P. Boyd (2000), Chebyshev and Fourier Spectral Methods, 2nd edn, Dover.

L. Caffarelli, R. Kohn and L. Nirenberg (1982), 'Partial regularity of suitable weak solutions of the Navier-Stokes equations', Commun. Pure Appl. Math. 35, 771-831.

R. Caflisch (1993), 'Singularity formation for complex solutions of the 3D incompressible Euler equations', Physica D 67, 1-18.

C. Cao and E. S. Titi (2007), 'Global well-posedness of the three-dimensional primitive equations of large scale ocean and atmosphere dynamics', Ann. of Math. 166, 245-267.

D. Chae (2006), 'Global regularity of the 2d Boussinesq equation with partial viscous terms', Adv. Math. 203, 497-513.

D. Chae and J. Lee (2002), 'On the regularity of the axisymmetric solutions of the Navier-Stokes equations', Math. Z. 239, 645-671.

C. Chen, R. M. Strain, T. Tsai and H. T. Yau (2008), 'Lower bound on the blowup rate of the axisymmetric Navier-Stokes equations', Int. Math. Res. Not., article ID rnn016.

C. Chen, R. M. Strain, T. Tsai and H. T. Yau (2009), 'Lower bound on the blowup rate of the axisymmetric Navier-Stokes equations II', to appear in Comm. Partial Differential Equations. 
A. Chorin (1982), 'The evolution of a turbulent vortex', Comm. Math. Phys. 83, 517 .

A. J. Chorin and J. E. Marsden (1993), A Mathematical Introduction to Fluid Mechanics, 3rd edn, Springer.

P. Constantin (1986), 'Note on loss of regularity for solutions of the 3-D incompressible Euler and related equations', Comm. Math. Phys. 104, 311-326.

P. Constantin (1994), 'Geometric statistics in turbulence', SIAM Review 36, 73.

P. Constantin and C. Foias (1988), Navier-Stokes Equations, Chicago University Press.

P. Constantin, C. Fefferman and A. Majda (1996), 'Geometric constraints on potentially singular solutions for the 3-D Euler equation', Comm. Partial Differential Equations 21, 559-571.

P. Constantin, P. D. Lax and A. Majda (1985), 'A simple one-dimensional model for the three-dimensional vorticity equations', Commun. Pure Appl. Math. 38, $715-724$.

J. Deng, T. Y. Hou and X. Yu (2005), 'Geometric properties and non-blowup of 3-D incompressible Euler flow', Comm. Partial Differential Equations 30, $225-243$.

J. Deng, T. Y. Hou and X. Yu (2006a), 'Improved geometric conditions for nonblowup of 3D incompressible Euler equation', Comm. Partial Differential Equations 31, 293-306.

J. Deng, T. Y. Hou, R. Li and X. Yu (2006b), 'Level set dynamics and the nonblow-up of the quasi-geostrophic equation', Methods Appl. Anal. 13, 157-180.

V. M. Fernandez, N. J. Zabusky and V. M. Gryanik (1995), 'Vortex intensification and collapse of the Lissajous-elliptic ring: Single and multi-filament BiotSavart simulations and visiometrics', J. Fluid Mech. 299, 289-331.

J. Goodman, T. Y. Hou and E. Tadmor (1994), 'On the stability of the unsmoothed Fourier method for hyperbolic equations', Numer. Math. 67, 93-129.

R. Grauer and T. Sideris (1991), 'Numerical computation of three dimensional incompressible ideal fluids with swirl', Phys. Rev. Lett. 67, 3511.

R. Grauer, C. Marliani and K. Germaschewski (1998), 'Adaptive mesh refinement for singular solutions of the incompressible Euler equations', Phys. Rev. Lett. $\mathbf{8 0}, 19$.

T. Y. Hou and Z. Lei $(2009 a)$, 'On the partial regularity of a 3D model of the Navier-Stokes equations', Comm. Math. Phys. 287, 589-612.

T. Y. Hou and Z. Lei $(2009 b)$, 'On the stabilizing effect of convection for three dimensional incompressible flows', Commun. Pure Appl. Math. 62, 501-564

T. Y. Hou and C. Li (2005), 'Global well-posedness of the viscous Boussinesq equations', Discrete Contin. Dyn. Syst. 12, 1-12.

T. Y. Hou and C. Li (2008a), 'Dynamic stability of the 3D axi-symmetric NavierStokes equations with swirl', Commun. Pure Appl. Math. 61, 661-697.

T. Y. Hou and R. Li (2006), 'Dynamic depletion of vortex stretching and nonblowup of the 3-D incompressible Euler equations', J. Nonlinear Sci. 16, 639664.

T. Y. Hou and R. Li (2007), 'Computing nearly singular solutions using pseudospectral methods', J. Comput. Phys. 226, 379-397. 
T. Y. Hou and R. Li (2008b), 'Blowup or no blowup? The interplay between theory and numerics', Physica D 237, 1937-1944.

T. Y. Hou, Z. Lei and C. Li (2008), 'Global regularity of the 3D axi-symmetric Navier-Stokes equations with anisotropic data', Comm. Partial Differential Equations 33, 1622-1637.

L. Iskauriaza, G. Seregin and V. Sverak (2003), ' $L_{3, \infty}$-solutions of Navier-Stokes equations and backward uniqueness', Uspekhi Mat. Nauk 58, 3-44.

R. M. Kerr (1993), 'Evidence for a singularity of the three dimensional, incompressible Euler equations', Phys. Fluids 5, 1725-1746.

R. M. Kerr (2005), 'Velocity and scaling of collapsing Euler vortices', Phys. Fluids 17, 075103-114.

R. M. Kerr and F. Hussain (1989), 'Simulation of vortex reconnection', Physica D 37, 474.

G. Koch, N. Nadirashvili, G. Seregin and V. Sverak (2009), 'Liouville theorems for the Navier-Stokes equations and applications', to appear in Acta Mathemat$i c a$.

H. Kozono and Y. Taniuchi (2000), 'Bilinear estimates in BMO and Navier-Stokes equations', Math Z. 235, 173-194.

O. Ladyzhenskaya (1970), Mathematical Problems of the Dynamics of Viscous Incompressible Fluids, Nauka, Moscow.

R. J. LeVeque (1992), Numerical Method for Conservation Laws, Birkhäuser.

F. H. Lin (1998), 'A new proof of the Caffarelli-Kohn-Nirenberg theorem', Commun. Pure Appl. Math. 51, 241-257.

J. G. Liu and W. C. Wang (2006), 'Convergence analysis of the energy and helicity preserving scheme for axisymmetric flows', SIAM J. Numer. Anal. 44, 24562480.

A. J. Majda and A. L. Bertozzi (2002), Vorticity and Incompressible Flow, Cambridge University Press.

R. B. Pelz (1997), 'Locally self-similar, finite-time collapse in a high-symmetry vortex filament model', Phys. Rev. E 55, 1617-1626.

G. Prodi (1959), 'Un teorema di unicità per el equazioni di Navier-Stokes', Ann. Mat. Pura Appl. 48, 173-182.

A. Pumir and E. E. Siggia (1990), 'Collapsing solutions to the 3-D Euler equations', Phys. Fluids A 2, 220-241.

G. Raugel and G. Sell (1993a), 'Navier-Stokes equations on thin 3D domains I: Global attractors and global regularity of solutions', J. Amer. Math. Soc. 6, 503-568.

G. Raugel and G. Sell (1993b), Navier-Stokes equations on thin 3D domains III: Global and local attractors, in Turbulence in Fluid Flows: A Dynamical Systems Approach, Vol. 55 of IMA Volumes in Mathematics and its Applications, Springer, pp. 137-163.

G. Raugel and G. Sell (1994), Navier-Stokes equations on thin 3D domains II: Global regularity of spatially periodic solutions, in Nonlinear Partial Differential Equations and their Applications, Collège de France Seminar, Vol. XI of Pitman Research Notes Math, Series 299, Longman, pp. 205-247.

J. Serrin (1962), 'On the interior regularity of weak solutions of the Navier-Stokes equations', Arch. Ration. Mech. Anal. 9, 187-195. 
J. Serrin (1963), The initial value problem for the Navier-Stokes equations, in Nonlinear Problems (R. Langer, ed.), University of Wisconsin Press, pp. 6998.

M. J. Shelley, D. I. Meiron and S. A. Orszag (1993), 'Dynamical aspects of vortex reconnection of perturbed anti-parallel vortex tubes', J. Fluid Mech. 246, 613652.

M. Struwe (1988), 'On partial regularity results for the Navier-Stokes equations', Commun. Pure Appl. Math. 41, 437-458.

R. Temam (2001), Navier-Stokes Equations, AMS, Providence, RI.

Online reference

C. Fefferman, Clay Mathematical Institute, Millennium Open Problems: www.claymath.org/millennium/Navier-Stokes_Equations/ 